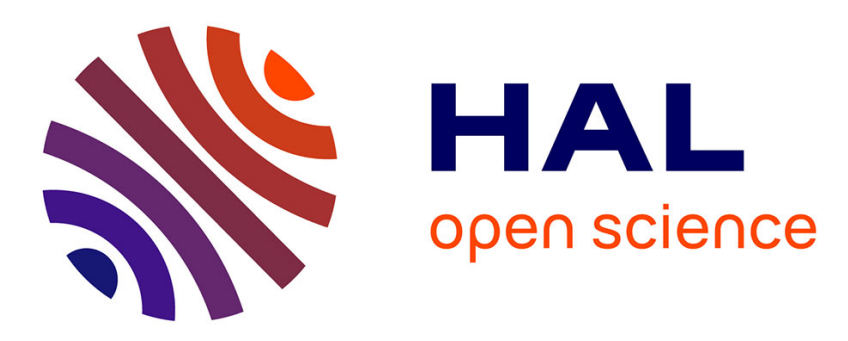

\title{
Convergence of monotone finite volume schemes for hyperbolic scalar conservation laws with multiplicative noise
}

Caroline Bauzet, J. Charrier, T. Gallouët

\section{- To cite this version:}

Caroline Bauzet, J. Charrier, T. Gallouët. Convergence of monotone finite volume schemes for hyperbolic scalar conservation laws with multiplicative noise. Stochastics and Partial Differential Equations: Analysis and Computations, 2016, 4 (1), pp.150-223. 10.1007/s40072-015-0052-z . hal-01061019v3

\section{HAL Id: hal-01061019 \\ https://hal.science/hal-01061019v3}

Submitted on 5 Jul 2016

HAL is a multi-disciplinary open access archive for the deposit and dissemination of scientific research documents, whether they are published or not. The documents may come from teaching and research institutions in France or abroad, or from public or private research centers.
L'archive ouverte pluridisciplinaire HAL, est destinée au dépôt et à la diffusion de documents scientifiques de niveau recherche, publiés ou non, émanant des établissements d'enseignement et de recherche français ou étrangers, des laboratoires publics ou privés. 


\title{
Convergence of monotone finite volume schemes for hyperbolic scalar conservation laws with multiplicative noise*
}

\author{
C. Bauzet ${ }^{\dagger}$, J. Charrier ${ }^{\dagger}$ and T. Gallouët ${ }^{\dagger}$
}

June 20, 2016

\begin{abstract}
We study here the discretization by monotone finite volume schemes of multi-dimensional nonlinear scalar conservation laws forced by a multiplicative noise with a time and space dependent flux-function and a given initial data in $L^{2}\left(\mathbb{R}^{d}\right)$. After establishing the well-posedness theory for solutions of such kind of stochastic problems, we prove under a stability condition on the time step the convergence of the finite volume approximation towards the unique stochastic entropy solution of the equation.
\end{abstract}

Keywords : Stochastic PDE • first-order hyperbolic equation $\bullet$ Itô integral $\bullet$ multiplicative noise $\bullet$ finite volume method $\bullet$ monotone scheme $\bullet$ Godunov scheme $\bullet$ Young measures $\bullet$ Kruzhkov smooth entropy.

Mathematics Subject Classification (2000) : 35L60 • 60H15 • 35L60

\section{Introduction}

We are interested in the Cauchy problem for a nonlinear hyperbolic scalar conservation law in $d$ space dimensions with a multiplicative stochastic perturbation of type:

$$
\left\{\begin{aligned}
d u+\operatorname{div}[\vec{v}(x, t) f(u)] d t & =g(u) d W \quad \text { in } \Omega \times \mathbb{R}^{d} \times(0, T), \\
u(\omega, x, 0) & =u_{0}(x), \quad \omega \in \Omega, x \in \mathbb{R}^{d}
\end{aligned}\right.
$$

where div is the divergence operator with respect to the space variable (which belongs to $\mathbb{R}^{d}$ ), $d$ is a positive integer, $T>0$ and $W=\left\{W_{t}, \mathcal{F}_{t} ; 0 \leqslant t \leqslant T\right\}$ is a standard adapted one-dimensional continuous Brownian motion defined on the classical Wiener space $(\Omega, \mathcal{F}, P)$. As mentioned by KIm Kim06, by denoting $Q=\mathbb{R}^{d} \times(0, T)$ this equation has to be understood in the following way: for almost all $\omega$ in $\Omega$ and for all $\varphi$ in $\mathcal{D}\left(\mathbb{R}^{d} \times[0, T)\right)$

$$
\begin{aligned}
& \int_{\mathbb{R}^{d}} u_{0}(x) \varphi(x, 0) d x+\int_{Q} u(\omega, x, t) \partial_{t} \varphi(x, t)+\vec{v}(x, t) f(u(\omega, x, t)) \cdot \nabla_{x} \varphi(x, t) d x d t \\
= & \int_{Q} \int_{0}^{t} g(u(\omega, x, s)) d W(s) \partial_{t} \varphi(x, t) d x d t .
\end{aligned}
$$

In order to relieve the presentation of the paper, we omit in the sequel the variables $\omega, x, t$ and write $u$ instead of $u(\omega, x, t)$.

Note that, even in the deterministic case, a weak solution to a nonlinear scalar conservation law is not unique in general. The mathematical challenge consists in introducing a selection criterion in order to identify a unique solution. In the present work we consider a stochastic version of the entropy condition proposed by KRUZHKOV in the 70s, the one used in BVW12 and adapted to a space and time dependent flux-function, which is presented in Section 2

We assume the following hypotheses:

$$
\begin{aligned}
& \mathrm{H}_{1}: u_{0} \in L^{2}\left(\mathbb{R}^{d}\right) . \\
& \mathrm{H}_{2}: \quad f: \mathbb{R} \rightarrow \mathbb{R} \text { is a Lipschitz-continuous function with } f(0)=0 . \\
& \mathrm{H}_{3}: g: \mathbb{R} \rightarrow \mathbb{R} \text { is a Lipschitz-continuous function with } g(0)=0 . \\
& \mathrm{H}_{4}: \vec{v} \in \mathcal{C}^{1}\left(\mathbb{R}^{d} \times[0, T], \mathbb{R}^{d}\right) \text { and } \operatorname{div}[\vec{v}(x, t)]=0 \forall(x, t) \in \mathbb{R}^{d} \times[0, T] .
\end{aligned}
$$

\footnotetext{
* This work has been carried out in the framework of the Labex Archimède (ANR-11-LABX-0033) and of the A*MIDEX project (ANR-11-IDEX-0001-02), funded by the "Investissements d'Avenir" French Government programme managed by the French National Research Agency (ANR)

†Aix-Marseille Université, CNRS, Centrale Marseille, I2M, UMR 7373, 13453 Marseille France, \{caroline.bauzet, julia.charrier, thierry.gallouet\}@univ-amu.fr
} 
$\mathrm{H}_{5}$ : There exists $V<\infty$ such that $|\vec{v}(x, t)| \leqslant V \forall(x, t) \in \mathbb{R}^{d} \times[0, T]$.

$\mathrm{H}_{6}: g$ is a bounded function.

Remark 1 (On these assumptions)

- $H_{1}$ to $H_{5}$ are used in the present work to prove the well-posedness of Problem (1). Note that, as it is classically done for hyperbolic scalar conservation laws, for convenience one can assume that $f(0)=0$ without loss of generality.

- $g(0)=0$ is a technical condition which allows us to show the well-posedness of our problem and is also used in the present work to show a priori estimates on the finite volume approximate solution.

- Note that the present study can be extended to the case $\operatorname{div}[\vec{v}(x, t)] \neq 0$ (which only brings technical difficulties) following for example the work of [CHOD] in the deterministic case.

- $H_{6}$ is used to show the convergence of the finite volume scheme (precisely to prove that the term denoted $S_{2}^{h, k}$ goes to 0 in the proof of Proposition 4).

Remark 2 Following VALLET Val08] Section 6.1, if we assume in addition the following hypotheses

(i) $u_{0} \in L^{\infty}\left(\mathbb{R}^{d}\right)$.

(ii) suppg $\subset[0,1]$.

then we can show that the stochastic entropy solution $u$ also belongs to $L^{\infty}\left(\mathbb{R}^{d}\right)$. Indeed, thanks to the Itô formula, this maximum principle is direct for the viscous solution $u_{\epsilon}$, then it is conserved at the limit for $u$. Note that assuming $(i)$ and (ii) allows us to treat the cases where $f$ and $g$ are only locally Lipschitzcontinuous. In particular, all the results stated in this paper hold if one considers the stochastic Burgers equation (i.e. when $f(u)=u^{2}$ ).

\subsection{State of the art}

Only few papers have been devoted to the theoretical study of scalar conservation laws with a multiplicative stochastic forcing, let us mention in chronological order the contributions of [FN08, [DV10], CDK12, [BVW12, BVW14, BM14], Hof14]. Concerning the study of numerical approximation of these stochastic problems, there is also, to our knowledge, few papers. Let us cite the work of [HR91 and also its recent generalization to the multidimensional-case [Bau14] where a time-discretization of the equation is proposed by the use of an operator-splitting method. Let us also mention the paper of KR12 where a spacediscretization of the equation is investigated by considering monotone numerical fluxes. In a recent submitted work, [BCG] proposed a time and space discretization of the problem and showed the convergence of a class of flux-splitting finite volume schemes towards the unique stochastic entropy solution of the problem by using the theoretical framework of BVW12. For a thorough exposition of all these papers, we refer the reader to the introduction of $[\mathrm{BCG}$. Note that to the best of our knowledge, in the case of a space and time dependent flux-function, stochastic equations of type (1) have not been studied yet from a theoretical (respectively numerical) point of view, neither by means of entropy formulation (respectively finite volume) framework nor by any other approachs.

\subsection{Goal of the study and outline of the paper}

The aim of the present paper is to fill the gap left by the previous authors by introducing a convergence result for a both time and space discretization of multi-dimensional nonlinear scalar conservation laws forced by a multiplicative noise and with a time and space dependent flux-function. More precisely, we firstly show that under assumptions $H_{1}$ to $H_{5}$, Problem (1) is well-posed. Secondly, we introduce a general finite volume monotone scheme for the discretization of such a problem and, by assuming additionally that hypothesis $H_{6}$ holds, we prove that the associated finite volume approximate solution converges in $L_{l o c}^{p}(\Omega \times Q)$ for all $1 \leqslant p<2$ to the unique stochastic entropy solution of the equation.

The paper is organized as follows. In Section 2 by adapting the work of BVW12 to the case of a time and space dependent flux-function, we propose the definition of a stochastic entropy solution for (1) and state the well-posedness result of the problem. For the sake of clarity, the proof of this theoretical result is presented in Appendix A. In Section 3 we define the general monotone scheme used to approximate the stochastic entropy solution of (1). Then, we give the main result of this paper, which states the convergence of the approximate solution towards the unique stochastic entropy solution of the equation. The remainder of the paper is devoted to the proof of this convergence result. In Section 4 , several preliminary results satisfied by the finite volume approximate solution denoted $u_{\mathcal{T}, k}$ are stated. Then, Section 5 is devoted to show the convergence of $u_{\mathcal{T}, k}$ towards the unique stochastic entropy solution of Problem (1). 


\subsection{General notations}

First of all, we need to introduce some notations and make precise the functional setting.

- $Q=\mathbb{R}^{d} \times(0, T)$.

- Throughout the paper, we denote by $C_{f}$ and $C_{g}$ the Lipschitz constants of $f$ and $g$.

- $|x|$ denotes the Euclidian norm of $x$ in $\mathbb{R}^{d}$ and $x . y$ the usual scalar product of $x$ and $y$ in $\mathbb{R}^{d}$.

- For $p=1, d$ or $d+1,\|\cdot\|_{\infty}$ denotes the $L^{\infty}\left(\mathbb{R}^{p}\right)$ norm.

- For any $p \geqslant 1, L_{l o c}^{p}(\Omega \times Q)$ denotes the set of measurable functions $f$ such that for any compact subset $K$ of $\mathbb{R}^{d}, f \in L^{p}(\Omega \times K \times(0, T))$.

- $E[$.$] denotes the expectation, i.e. the integral over \Omega$ with respect to the probability measure $P$.

- $\mathcal{D}^{+}\left(\mathbb{R}^{d} \times[0, T)\right)$ denotes the subset of nonnegative elements of $\mathcal{D}\left(\mathbb{R}^{d} \times[0, T)\right)$.

- For a given separable Banach space $X$ we denote by $\mathcal{N}_{w}^{2}(0, T, X)$ the space of the predictable $X$-valued processes endowed with the norm $\|\phi\|_{\mathcal{N}_{w}^{2}(0, T, X)}^{2}:=E\left[\int_{0}^{T}\|\phi\|_{X}^{2} d t\right]$ (see DPZ92 p.94).

- $\mathcal{A}$ denotes the set of any $C^{3}(\mathbb{R})$ convex functions such that $\eta^{\prime}, \eta^{\prime \prime}$ and $\eta^{\prime \prime \prime}$ are bounded functions.

- $\Phi$ denotes the entropy flux defined for any $a \in \mathbb{R}$ and for any smooth function $\eta \in \mathcal{A}$ by $\Phi(a)=\int_{0}^{a} \eta^{\prime}(\sigma) f^{\prime}(\sigma) d \sigma$. Note in particular that $\Phi$ is a Lipschitz-continuous function.

\section{The continuous problem}

Let us introduce in this section the definition of a solution for Problem (1) and the existence and uniqueness result which ensures us the well-posedness of such a problem. This result is obtained under hypotheses $H_{1}$ to $H_{5}$ and is adapted from the work of [BVW12.

Definition 1 (Stochastic entropy solution)

A function $u$ of $\mathcal{N}_{w}^{2}\left(0, T, L^{2}\left(\mathbb{R}^{d}\right)\right) \cap L^{\infty}\left(0, T ; L^{2}\left(\Omega \times \mathbb{R}^{d}\right)\right)$ is an entropy solution of the stochastic scalar conservation law (1) with the initial condition $u_{0} \in L^{2}\left(\mathbb{R}^{d}\right)$, if $P$-a.s in $\Omega$, for any $\eta \in \mathcal{A}$ and for any $\varphi \in \mathcal{D}^{+}\left(\mathbb{R}^{d} \times[0, T)\right)$

$$
\begin{aligned}
0 \leqslant & \int_{\mathbb{R}^{d}} \eta\left(u_{0}\right) \varphi(x, 0) d x+\int_{Q} \eta(u) \partial_{t} \varphi(x, t) d x d t+\int_{Q} \Phi(u) \vec{v}(x, t) \cdot \nabla_{x} \varphi(x, t) d x d t \\
& +\int_{0}^{T} \int_{\mathbb{R}^{d}} \eta^{\prime}(u) g(u) \varphi(x, t) d x d W(t)+\frac{1}{2} \int_{Q} g^{2}(u) \eta^{\prime \prime}(u) \varphi(x, t) d x d t
\end{aligned}
$$

For technical reasons, as in BVW12 and BCG, we also need to consider a generalized notion of entropy solution. In fact, in a first step, we will only prove the convergence of the finite volume approximate solution $u_{\mathcal{T}, k}$ to a measure-valued entropy solution. Then, thanks to the result of uniqueness stated in Theorem 1 . we will be able to deduce the convergence of $u_{\mathcal{T}, k}$ to the unique stochastic entropy solution of Problem (1).

\section{Definition 2 (Measure-valued entropy solution)}

A function $\mathbf{u}$ of $\mathcal{N}_{w}^{2}\left(0, T, L^{2}\left(\mathbb{R}^{d} \times(0,1)\right)\right) \cap L^{\infty}\left(0, T ; L^{2}\left(\Omega \times \mathbb{R}^{d} \times(0,1)\right)\right)$ is a measure-valued entropy solution of the stochastic scalar conservation law (1) with the initial condition $u_{0} \in L^{2}\left(\mathbb{R}^{d}\right)$, if P-a.s in $\Omega$, for any $\eta \in \mathcal{A}$ and for any $\varphi \in \mathcal{D}^{+}\left(\mathbb{R}^{d} \times[0, T)\right)$

$$
\begin{aligned}
0 & \leqslant \int_{\mathbb{R}^{d}} \eta\left(u_{0}\right) \varphi(x, 0) d x+\int_{Q} \int_{0}^{1} \eta(\mathbf{u}(., \alpha)) \partial_{t} \varphi(x, t) d \alpha d x d t+\int_{Q} \int_{0}^{1} \Phi(\mathbf{u}(., \alpha)) \vec{v}(x, t) \cdot \nabla_{x} \varphi(x, t) d \alpha d x d t \\
& +\int_{0}^{T} \int_{\mathbb{R}^{d}} \int_{0}^{1} \eta^{\prime}(\mathbf{u}(., \alpha)) g(\mathbf{u}(., \alpha)) \varphi(x, t) d \alpha d x d W(t)+\frac{1}{2} \int_{Q} \int_{0}^{1} g^{2}(\mathbf{u}(., \alpha)) \eta^{\prime \prime}(\mathbf{u}(., \alpha)) \varphi(x, t) d \alpha d x d t .
\end{aligned}
$$

Theorem 1 Under assumptions $H_{1}$ to $H_{5}$ there exists a unique measure-valued entropy solution for the Problem (1) and this solution is obtained by viscous approximation. Moreover, it is the unique stochastic entropy solution in the sense of Definition 1 .

Remark 3 The proof of this theorem is presented in Appendix A. The existence proof relies on a parabolic regularization of (1) and the uniqueness result is obtained by adapting the Kruzhkov's doubling variable technique of the deterministic setting to the stochastic case as it is done in [BVW12].

\section{Main result}

In the sequel, assume that assumptions $H_{1}$ to $H_{6}$ hold. Let us first give a definition of the admissible meshes for the finite volume scheme. 


\subsection{Meshes and scheme}

Definition 3 (Admissible mesh) An admissible mesh $\mathcal{T}$ of $\mathbb{R}^{d}$ for the discretization of Problem (1) is given by a family of disjoint polygonal connected subset of $\mathbb{R}^{d}$ such that $\mathbb{R}^{d}$ is the union of the closure of the elements of $\mathcal{T}$ (which are called control volumes in the following) and such that the common interface of any two control volumes is included in a hyperplane of $\mathbb{R}^{d}$. It is assumed that $h=\operatorname{size}(\mathcal{T})=\sup \{\operatorname{diam}(K), K \in$ $\mathcal{T}\}<\infty$ and that, for some $\bar{\alpha} \in \mathbb{R}_{+}^{\star}$, we have

$$
\bar{\alpha} h^{d} \leqslant|K|, \quad \text { and } \quad|\partial K| \leqslant \frac{1}{\bar{\alpha}} h^{d-1}, \quad \forall K \in \mathcal{T}
$$

where we denote by

. $\mathcal{E}$ the set of all the interfaces of the mesh $\mathcal{T}$.

. $\partial K$ the boundary of the control volume $K$.

- $|K|$ the d-dimensional Lebesgue measure of $K$.

- $|\partial K|$ the $(d-1)$-dimensional Lebesgue measure of $\partial K$.

. $\mathcal{E}_{K}$ the set of interfaces of the control volume $K$.

. $\mathcal{N}(K)$ the set of control volumes neighbors of the control volume $K$.

- $\sigma_{K, L}$ the common interface between $K$ and $L$ for any $L \in \mathcal{N}(K)$.

- $n_{K, L}$ the unit normal vector to interface $\sigma_{K, L}$, oriented from $K$ to $L$, for any $L \in \mathcal{N}(K)$.

From (3) we get the following inequality, which will be used several times later :

$$
\frac{|\partial K|}{|K|} \leqslant \frac{1}{\bar{\alpha}^{2} h}
$$

We now define the general monotone scheme. Consider an admissible mesh $\mathcal{T}$ in the sense of Definition 3 In order to compute an approximation of $u$ on $[0, T]$ we take $N \in \mathbb{N}^{\star}$ and define the time step $k=\frac{T}{N} \in \mathbb{R}_{+}^{\star}$. In this way $[0, T]=\bigcup_{n=0}^{N-1}[n k,(n+1) k]$.

The equations satisfied by the discrete unknowns denoted by $u_{K}^{n}, n \in\{0, \ldots, N-1\}, K \in \mathcal{T}$, are obtained by discretizing Problem (1). For the discretization of such a problem, we need to define the numerical flux.

Definition 4 (Monotone numerical flux) We say that a function $F \in \mathcal{C}\left(\mathbb{R}^{2}, \mathbb{R}\right)$ is a monotone numerical flux if it satisfies the following properties:

. $F(a, b)$ is nondecreasing with respect to $a$ and nonincreasing with respect to $b$.

. There exists $F_{1}, F_{2}>0$ such that for any $a, b \in \mathbb{R}$ we have $|F(b, a)-F(a, a)| \leq F_{1}|a-b|$ and $\mid F(a, b)$ $F(a, a)\left|\leq F_{2}\right| a-b \mid$

. $F(a, a)=f(a)$ for all $a \in \mathbb{R}$.

\section{Remark 4}

. It is not necessary to suppose $F$ to be continuous, even with respect to each variable separately.

. It is possible to choose a numerical flux $F$ depending on $\mathcal{T}, \sigma_{K, L}, n$, as soon as the constants $F_{1}, F_{2}$ can be chosen independently of $\mathcal{T}, \sigma_{K, L}, n$. For the sake of readability we will consider in what follows a numerical flux $F$ independent of $\mathcal{T}, K \mid L, n$.

The set $\left\{u_{K}^{0}, K \in \mathcal{T}\right\}$ is given by the initial condition

$$
u_{K}^{0}=\frac{1}{|K|} \int_{K} u_{0}(x) d x, \forall K \in \mathcal{T}
$$

The equations satisfied by the discrete unknowns $u_{K}^{n}, n \in\{0, \ldots, N-1\}, K \in \mathcal{T}$ are given by the following explicit scheme associated to any monotone numerical flux $F$ : for any $K \in \mathcal{T}$, any $n \in\{0, \ldots, N-1\}$

$$
\frac{|K|}{k}\left(u_{K}^{n+1}-u_{K}^{n}\right)+\sum_{L \in \mathcal{N}(K)}\left|\sigma_{K, L}\right|\left\{v_{K, L}^{n} F\left(u_{K}^{n}, u_{L}^{n}\right)-v_{L, K}^{n} F\left(u_{L}^{n}, u_{K}^{n}\right)\right\}=|K| g\left(u_{K}^{n}\right) \frac{W^{n+1}-W^{n}}{k}
$$

where, by denoting $d \gamma$ the $(d-1)$-dimensional Lebesgue measure

$$
\begin{gathered}
v_{K, L}^{n}=\frac{1}{k\left|\sigma_{K, L}\right|} \int_{n k}^{(n+1) k} \int_{\sigma_{K, L}}\left(\vec{v}(x, t) \cdot n_{K, L}\right)^{+} d \gamma(x) d t \\
v_{L, K}^{n}=\frac{1}{k\left|\sigma_{K, L}\right|} \int_{n k}^{(n+1) k} \int_{\sigma_{K, L}}\left(\vec{v}(x, t) \cdot n_{L, K}\right)^{+} d \gamma(x) d t=\frac{1}{k\left|\sigma_{K, L}\right|} \int_{n k}^{(n+1) k} \int_{\sigma_{K, L}}\left(\vec{v}(x, t) \cdot n_{K, L}\right)^{-} d \gamma(x) d t
\end{gathered}
$$


and $W^{n}=W(n k) \forall n \in\{0, \ldots, N-1\}$.

The approximate finite volume solution $u_{\mathcal{T}, k}$ may be defined on $\Omega \times \mathbb{R}^{d} \times[0, T)$ from the discrete unknowns $u_{K}^{n}, K \in \mathcal{T}, n \in\{0, \ldots, N-1\}$ which are computed in $(6)$ by:

$$
u_{\mathcal{T}, k}(\omega, x, t)=u_{K}^{n} \text { for } \omega \in \Omega, x \in K \text { and } t \in[n k,(n+1) k) .
$$

Remark 5 Note that

$$
\begin{array}{r}
v_{K, L}^{n}-v_{L, K}^{n}=\frac{1}{k\left|\sigma_{K, L}\right|} \int_{n k}^{(n+1) k} \int_{\sigma_{K, L}} \vec{v}(x, t) \cdot n_{K, L} d \gamma(x) d t \\
\text { and } v_{K, L}^{n}+v_{L, K}^{n}=\frac{1}{k\left|\sigma_{K, L}\right|} \int_{n k}^{(n+1) k} \int_{\sigma_{K, L}}\left|\vec{v}(x, t) \cdot n_{K, L}\right| d \gamma(x) d t .
\end{array}
$$

Moreover, since $\operatorname{div}[\vec{v}(x, t)]=0$ for any $(x, t) \in \mathbb{R}^{d} \times[0, T]$, we have

$$
\sum_{L \in \mathcal{N}(K)}\left|\sigma_{K, L}\right|\left(v_{K, L}^{n}-v_{L, K}^{n}\right)=0 .
$$

Indeed,

$$
\begin{aligned}
\sum_{L \in \mathcal{N}(K)}\left|\sigma_{K, L}\right|\left(v_{K, L}^{n}-v_{L, K}^{n}\right) & =\sum_{L \in \mathcal{N}(K)}\left|\sigma_{K, L}\right|\left(\frac{1}{k\left|\sigma_{K, L}\right|} \int_{n k}^{(n+1) k} \int_{\sigma_{K, L}} \vec{v}(x, t) \cdot n_{K, L} d \gamma(x) d t\right) \\
& =\frac{1}{k} \int_{n k}^{(n+1) k} \int_{K} \operatorname{div}[\vec{v}(x, t)] d x d t=0 .
\end{aligned}
$$

Remark 6 (On the measurability of the approximate finite volume solution) Let us mention that using properties of the Brownian motion, for all $K$ in $\mathcal{T}$ and all $n$ in $\{0, \ldots, N-1\}, u_{K}^{n}$ is $\mathcal{F}_{n k}$-measurable and so, as an elementary process adapted to the filtration $\left(\mathcal{F}_{t}\right)_{t \geqslant 0}, u_{\mathcal{T}, k}$ is predictable with values in $L^{2}\left(\mathbb{R}^{d}\right)$.

\subsection{Main result}

We now state the main result of this paper.

Theorem 2 (Convergence to the stochastic entropy solution) Assume that hypotheses $H_{1}$ to $H_{6}$ hold. Let $\mathcal{T}$ be an admissible mesh in the sense of Definition 3. $N \in \mathbb{N}^{\star}$ and $k=\frac{T}{N} \in \mathbb{R}_{+}^{\star}$ be the time step. Let $u_{\mathcal{T}, k}$ be the finite volume approximation defined by the monotone finite volume scheme (6) and (7). Then $u_{\mathcal{T}, k}$ converges to the unique stochastic entropy solution of 11) in the sense of Definition 1 , in $L_{\text {loc }}^{p}(\Omega \times Q)$ for any $p<2$ as $h$ tends to 0 and $k / h$ tends to 0 .

Remark 7 Under the CFL Condition

$$
k \leqslant(1-\xi) \frac{\bar{\alpha}^{2} h}{V\left(F_{1}+F_{2}\right)}
$$

one gets for $\xi=0$ the $L_{t}^{\infty} L_{\omega, x}^{2}$ stability of $u_{\mathcal{T}, k}$ stated in Proposition $1, p$, and for some $\xi \in(0,1)$ the "weak BV" estimate stated in Proposition 2 $p$ 9. In the deterministic case, condition (9) for some $\xi \in(0,1)$ is sufficient to show the convergence of $u_{\mathcal{T}, k}$ to the unique entropy solution of the problem, whereas in the stochastic case this condition doesn't seem to be sufficient to show the convergence of the scheme, that is why we assume the stronger assumption $k / h \rightarrow 0$ as $h \rightarrow 0$.

Remark 8 This theorem can easily be generalized to the case of a stochastic finite dimensional perturbation of the form $g(u) . d \boldsymbol{W}$ where $g$ takes values into $\mathbb{R}^{p}$ and $\boldsymbol{W}$ is a p-dimensional Brownian motion.

\section{Preliminary results on the finite volume approximation}

\subsection{Stability estimates}

Let us state several results on the finite volume approximate solution $u_{\mathcal{T}, k}$ defined by (6) and (7).

Proposition $1\left(L_{t}^{\infty} L_{\omega, x}^{2}\right.$ estimate) Let $T>0, u_{0} \in L^{2}\left(\mathbb{R}^{d}\right), \mathcal{T}$ be an admissible mesh in the sense of Definition 3, $N \in \mathbb{N}^{\star}$ and $k=\frac{T}{N} \in \mathbb{R}_{+}^{*}$ satisfying the Courant-Friedrichs-Levy (CFL) condition

$$
k \leqslant \frac{\bar{\alpha}^{2} h}{V\left(F_{1}+F_{2}\right)} .
$$


Let $u_{\mathcal{T}, k}$ be the finite volume approximate solution defined by (6) and (7).

Then we have the following bound

$$
\left\|u_{\tau, k}\right\|_{L^{\infty}\left(0, T ; L^{2}\left(\Omega \times \mathbb{R}^{d}\right)\right)} \leqslant e^{C_{g}^{2} T / 2}\left\|u_{0}\right\|_{L^{2}\left(\mathbb{R}^{d}\right)} .
$$

As a consequence we get

$$
\left\|u_{\mathcal{T}, k}\right\|_{L^{2}(\Omega \times Q)}^{2} \leqslant T e^{T C_{g}^{2}}\left\|u_{0}\right\|_{L^{2}\left(\mathbb{R}^{d}\right)}^{2} .
$$

Proof. Let us show by induction on $n \in\{0, . ., N-1\}$ the following property:

$$
\sum_{K \in \mathcal{T}}|K| E\left[\left(u_{K}^{n}\right)^{2}\right] \leqslant\left(1+k C_{g}^{2}\right)^{n}\left\|u_{0}\right\|_{L^{2}\left(\mathbb{R}^{d}\right)}^{2} .
$$

First one has

$$
\begin{aligned}
\sum_{K \in \mathcal{T}}|K| E\left[\left(u_{K}^{0}\right)^{2}\right] & =\sum_{K \in \mathcal{T}}|K| E\left[\left(\frac{1}{|K|} \int_{K} u_{0}(x) d x\right)^{2}\right] \\
& \leqslant\left\|u_{0}\right\|_{L^{2}\left(\mathbb{R}^{d}\right)}^{2} .
\end{aligned}
$$

Set $n \in\{0, \ldots, N-1\}$ and assume that $\left(P_{n}\right)$ holds. Let us multiply the numerical scheme $[6]$ by $u_{K}^{n}$, we thus get

$$
\begin{aligned}
\frac{|K|}{k}\left[u_{K}^{n+1}-u_{K}^{n}\right] u_{K}^{n}= & -\sum_{L \in \mathcal{N}(K)}\left|\sigma_{K, L}\right|\left\{v_{K, L}^{n} F\left(u_{K}^{n}, u_{L}^{n}\right)-v_{L, K}^{n} F\left(u_{L}^{n}, u_{K}^{n}\right)\right\} u_{K}^{n} \\
& +\frac{|K|}{k} g\left(u_{K}^{n}\right)\left(W^{n+1}-W^{n}\right) u_{K}^{n} .
\end{aligned}
$$

By using formula $a b=\frac{1}{2}\left[(a+b)^{2}-a^{2}-b^{2}\right]$ with $a=u_{K}^{n+1}-u_{K}^{n}$ and $b=u_{K}^{n}$ we obtain

$$
\begin{aligned}
\frac{1}{2} \frac{|K|}{k}\left[\left(u_{K}^{n+1}\right)^{2}-\left(u_{K}^{n}\right)^{2}-\left(u_{K}^{n+1}-u_{K}^{n}\right)^{2}\right]= & -\sum_{L \in \mathcal{N}(K)}\left|\sigma_{K, L}\right|\left\{v_{K, L}^{n} F\left(u_{K}^{n}, u_{L}^{n}\right)-v_{L, K}^{n} F\left(u_{L}^{n}, u_{K}^{n}\right)\right\} u_{K}^{n} \\
& +\frac{|K|}{k} g\left(u_{K}^{n}\right)\left(W^{n+1}-W^{n}\right) u_{K}^{n}
\end{aligned}
$$

and then

$$
\begin{aligned}
\frac{|K|}{2}\left[\left(u_{K}^{n+1}\right)^{2}-\left(u_{K}^{n}\right)^{2}\right]= & \frac{|K|}{2}\left(u_{K}^{n+1}-u_{K}^{n}\right)^{2}-k \sum_{L \in \mathcal{N}(K)}\left|\sigma_{K, L}\right|\left\{v_{K, L}^{n} F\left(u_{K}^{n}, u_{L}^{n}\right)-v_{L, K}^{n} F\left(u_{L}^{n}, u_{K}^{n}\right)\right\} u_{K}^{n} \\
& +|K| g\left(u_{K}^{n}\right)\left(W^{n+1}-W^{n}\right) u_{K}^{n} .
\end{aligned}
$$

Using the finite volume scheme (6) we can replace $\left(u_{K}^{n+1}-u_{K}^{n}\right)^{2}$ and we take then the expectation. Thanks to the independance between the random variables $\left(W^{n+1}-W^{n}\right)$ and $u_{K}^{n}$, together with the equality $E\left[\left(g\left(u_{K}^{n}\right)\left(W^{n+1}-W^{n}\right)\right)^{2}\right]=E\left[\left(g\left(u_{K}^{n}\right)\right)^{2}\right] E\left[\left(W^{n+1}-W^{n}\right)^{2}\right]=k E\left[\left(g\left(u_{K}^{n}\right)\right)^{2}\right]$, we get

$$
\begin{aligned}
\frac{|K|}{2} E\left[\left(u_{K}^{n+1}\right)^{2}-\left(u_{K}^{n}\right)^{2}\right]= & \frac{|K|}{2} E\left[\left(-\frac{k}{|K|} \sum_{L \in \mathcal{N}(K)}\left|\sigma_{K, L}\right|\left\{v_{K, L}^{n} F\left(u_{K}^{n}, u_{L}^{n}\right)-v_{L, K}^{n} F\left(u_{L}^{n}, u_{K}^{n}\right)\right\}+g\left(u_{K}^{n}\right)\left(W^{n+1}-W^{n}\right)\right)^{2}\right] \\
& -k E\left[\sum_{L \in \mathcal{N}(K)}\left|\sigma_{K, L}\right|\left\{v_{K, L}^{n} F\left(u_{K}^{n}, u_{L}^{n}\right)-v_{L, K}^{n} F\left(u_{L}^{n}, u_{K}^{n}\right)\right\} u_{K}^{n}\right]+|K| E\left[g\left(u_{K}^{n}\right)\left(W^{n+1}-W^{n}\right) u_{K}^{n}\right] \\
= & \left.\frac{k^{2}}{2|K|} E\left[\sum_{L \in \mathcal{N}(K)}\left|\sigma_{K, L}\right|\left\{v_{K, L}^{n} F\left(u_{K}^{n}, u_{L}^{n}\right)-v_{L, K}^{n} F\left(u_{L}^{n}, u_{K}^{n}\right)\right\}\right)^{2}\right]+\frac{k|K|}{2} E\left[\left(g\left(u_{K}^{n}\right)\right)^{2}\right] \\
& -k E\left[\sum_{L \in \mathcal{N}(K)}\left|\sigma_{K, L}\right|\left\{v_{K, L}^{n} F\left(u_{K}^{n}, u_{L}^{n}\right)-v_{L, K}^{n} F\left(u_{L}^{n}, u_{K}^{n}\right)\right\} u_{K}^{n}\right]
\end{aligned}
$$

Using $\sqrt{8}$, which states that $\sum_{L \in \mathcal{N}(K)}\left|\sigma_{K, L}\right|\left(v_{K, L}^{n}-v_{L, K}^{n}\right)=0$, this equality can be rewritten as

$$
\frac{|K|}{2} E\left[\left(u_{K}^{n+1}\right)^{2}-\left(u_{K}^{n}\right)^{2}\right]=B_{1}-B_{2}+D
$$




$$
\begin{aligned}
\text { where } \quad B_{1} & =\frac{k^{2}}{2|K|} E\left[\left(\sum_{L \in \mathcal{N}(K)}\left|\sigma_{K, L}\right|\left\{v_{K, L}^{n}\left(F\left(u_{K}^{n}, u_{L}^{n}\right)-f\left(u_{K}^{n}\right)\right)-v_{L, K}^{n}\left(F\left(u_{L}^{n}, u_{K}^{n}\right)-f\left(u_{K}^{n}\right)\right)\right\}\right)^{2}\right] \\
B_{2} & =k E\left[\sum_{L \in \mathcal{N}(K)}\left|\sigma_{K, L}\right|\left\{v_{K, L}^{n}\left(F\left(u_{K}^{n}, u_{L}^{n}\right)-f\left(u_{K}^{n}\right)\right)-v_{L, K}^{n}\left(F\left(u_{L}^{n}, u_{K}^{n}\right)-f\left(u_{K}^{n}\right)\right)\right\} u_{K}^{n}\right] \\
\text { and } \quad D & =\frac{k|K|}{2} E\left[\left(g\left(u_{K}^{n}\right)\right)^{2}\right] .
\end{aligned}
$$

Let us now define $B_{3}$ by

$$
\begin{aligned}
B_{3}=k \sum_{(K, L) \in \mathfrak{T}_{n}}\left|\sigma_{K, L}\right| E[ & v_{K, L}^{n}\left\{u_{K}^{n}\left(F\left(u_{K}^{n}, u_{L}^{n}\right)-f\left(u_{K}^{n}\right)\right)-u_{L}^{n}\left(F\left(u_{K}^{n}, u_{L}^{n}\right)-f\left(u_{L}^{n}\right)\right)\right\} \\
& \left.-v_{L, K}^{n}\left\{u_{K}^{n}\left(F\left(u_{L}^{n}, u_{K}^{n}\right)-f\left(u_{K}^{n}\right)\right)-u_{L}^{n}\left(F\left(u_{L}^{n}, u_{K}^{n}\right)-f\left(u_{L}^{n}\right)\right)\right\}\right]
\end{aligned}
$$

where $\mathfrak{T}_{n}=\left\{(K, L) \in \mathcal{T}^{2}: L \in \mathcal{N}(K)\right.$ and $\left.u_{K}^{n}>u_{L}^{n}\right\}$. One notes that $\sum_{K \in \mathcal{T}} B_{2}=B_{3}$.

Denoting by $\phi$ the function defined for any $a \in \mathbb{R}$ by $\phi(a)=\int_{0}^{a} s f^{\prime}(s) d s$, an integration by parts yields, for all $(a, b) \in \mathbb{R}^{2}$

$$
\phi(b)-\phi(a)=\int_{a}^{b} s f^{\prime}(s) d s=b(f(b)-F(a, b))-a(f(a)-F(a, b))-\int_{a}^{b}(f(s)-F(a, b)) d s .
$$

By using this formula, the term $B_{3}$ can be decomposed as $B_{3}=B_{4}-B_{5}$ where

$$
B_{4}=E\left[\sum_{(K, L) \in \mathfrak{T}_{n}} k\left|\sigma_{K, L}\right|\left\{v_{K, L}^{n}\left(\int_{u_{K}^{n}}^{u_{L}^{n}}\left(f(s)-F\left(u_{K}^{n}, u_{L}^{n}\right)\right) d s\right)+v_{L, K}^{n}\left(\int_{u_{L}^{n}}^{u_{K}^{n}}\left(f(s)-F\left(u_{L}^{n}, u_{K}^{n}\right)\right) d s\right)\right\}\right]
$$

and

$$
B_{5}=E\left[\sum_{(K, L) \in \mathfrak{T}_{n}} k\left|\sigma_{K, L}\right|\left(v_{K, L}^{n}-v_{L, K}^{n}\right)\left\{\phi\left(u_{K}^{n}\right)-\phi\left(u_{L}^{n}\right)\right\}\right] .
$$

Note that since $\operatorname{div}[\vec{v}(x, t)]=0 \forall(x, t) \in \mathbb{R}^{d} \times[0, T], B_{5}=0$. Indeed,

$$
\begin{aligned}
B_{5}=E\left[\sum_{(K, L) \in \mathfrak{I}_{n}} k\left|\sigma_{K, L}\right|\left(\frac{1}{k\left|\sigma_{K, L}\right|} \int_{n k}^{(n+1) k} \int_{\sigma_{K, L}} \vec{v}(x, t) \cdot n_{K, L} d \gamma(x) d t\right)\left\{\phi\left(u_{K}^{n}\right)-\phi\left(u_{L}^{n}\right)\right\}\right] \\
=E\left[\sum _ { ( K , L ) \in \mathfrak { I } _ { n } } \left\{\left(\int_{n k}^{(n+1) k} \int_{\sigma_{K, L}} \vec{v}(x, t) \cdot n_{K, L} d \gamma(x) d t\right) \phi\left(u_{K}^{n}\right)\right.\right. \\
\left.\left.\quad+\left(\int_{n k}^{(n+1) k} \int_{\sigma_{K, L}} \vec{v}(x, t) \cdot n_{L, K} d \gamma(x) d t\right) \phi\left(u_{L}^{n}\right)\right\}\right] \\
=E\left[\sum_{K \in \mathcal{T}} \sum_{L \in \mathcal{N}(K)} \phi\left(u_{K}^{n}\right) \int_{n k}^{(n+1) k} \int_{\sigma_{K, L}} \vec{v}(x, t) \cdot n_{K, L} d \gamma(x) d t\right] \\
=E\left[\sum_{K \in \mathcal{T}} \phi\left(u_{K}^{n}\right) \int_{n k}^{(n+1) k} \int_{K} \operatorname{div}[\vec{v}(x, t)] d x d t\right] \\
=0 .
\end{aligned}
$$

Let us now turn to an estimate of $B_{4}$.

We now use the following technical lemma from [EGH00] (Lemma 4.5 p.107):

Lemma 1 Let $\mathcal{G}: \mathbb{R} \rightarrow \mathbb{R}$ be a monotone Lipschitz-continuous function with a Lipschitz constant $C_{\mathcal{G}}>0$. Then:

$$
\left|\int_{c}^{d} \mathcal{G}(t)-\mathcal{G}(c) d t\right| \geqslant \frac{1}{2 C_{\mathcal{G}}}(\mathcal{G}(d)-\mathcal{G}(c))^{2}, \forall c, d \in \mathbb{R} .
$$

From this lemma, we can notice that for all $a, b \in \mathbb{R}$ we have

$$
\begin{aligned}
\int_{a}^{b} f(t)-F(a, b) d t & \geqslant \int_{a}^{b} F(a, t)-F(a, a) d t \geqslant \frac{1}{2 F_{2}}(f(a)-F(a, b))^{2} \\
\text { and } & \\
\int_{a}^{b} f(t)-F(a, b) d t & \geqslant \int_{a}^{b} F(t, b)-F(a, b) d t \geqslant \frac{1}{2 F_{1}}(f(b)-F(a, b))^{2}
\end{aligned}
$$


Multiplying 12 (respectively 13 ) by $\frac{F_{2}}{F_{1}+F_{2}}$ (respectively by $\frac{F_{1}}{F_{1}+F_{2}}$ ) and adding the two inequalities yields:

$$
\int_{a}^{b} f(t)-F(a, b) d t \geqslant \frac{1}{2\left(F_{1}+F_{2}\right)}\left[(f(a)-F(a, b))^{2}+(f(b)-F(a, b))^{2}\right] .
$$

We can deduce from this inequality that

$$
\begin{aligned}
B_{3}=B_{4} \geqslant k \sum_{(K, L) \in \mathfrak{T}_{n}}\left|\sigma_{K, L}\right| E & {\left[\frac{v_{K, L}^{n}}{2\left(F_{1}+F_{2}\right)}\left\{\left(F\left(u_{K}^{n}, u_{L}^{n}\right)-f\left(u_{K}^{n}\right)\right)^{2}+\left(F\left(u_{K}^{n}, u_{L}^{n}\right)-f\left(u_{L}^{n}\right)\right)^{2}\right\}\right.} \\
& \left.+\frac{v_{L, K}^{n}}{2\left(F_{1}+F_{2}\right)}\left\{\left(f\left(u_{K}^{n}\right)-F\left(u_{L}^{n}, u_{K}^{n}\right)\right)^{2}+\left(f\left(u_{L}^{n}\right)-F\left(u_{L}^{n}, u_{K}^{n}\right)\right)^{2}\right\}\right] .
\end{aligned}
$$

This gives finally a bound on $B_{3}$. Let us now turn to the study of $B_{1}$.

We have, after summing over $K \in \mathcal{T}$ :

$$
\sum_{K \in \mathcal{T}} B_{1}=\sum_{K \in \mathcal{T}} \frac{k^{2}}{2|K|} E\left[\left(\sum_{L \in \mathcal{N}(K)}\left|\sigma_{K, L}\right|\left\{v_{K, L}^{n}\left(F\left(u_{K}^{n}, u_{L}^{n}\right)-f\left(u_{K}^{n}\right)\right)-v_{L, K}^{n}\left(F\left(u_{L}^{n}, u_{K}^{n}\right)-f\left(u_{K}^{n}\right)\right)\right\}\right)^{2}\right] .
$$

Using the notations $A=F\left(u_{K}^{n}, u_{L}^{n}\right)-f\left(u_{K}^{n}\right), B=F\left(u_{L}^{n}, u_{K}^{n}\right)-f\left(u_{K}^{n}\right), \zeta=\frac{v_{K, L}^{n}}{v_{K, L}^{n}+v_{L, K}^{n}}$, $1-\zeta=\frac{v_{L, K}^{n}}{v_{K, L}^{n}+v_{L, K}^{n}}, \zeta \in(0,1)$ we get using Cauchy-Schwarz inequality that

$$
\begin{aligned}
\left(\sum_{L \in \mathcal{N}(K)} \mid\right. & \left.\sigma_{K, L} \mid\left\{v_{K, L}^{n} A-v_{L, K}^{n} B\right\}\right)^{2}=\left(\sum_{L \in \mathcal{N}(K)}\left|\sigma_{K, L}\right|\left(v_{K, L}^{n}+v_{L, K}^{n}\right)\{\zeta A-(1-\zeta) B\}\right)^{2} \\
& \leqslant \sum_{L \in \mathcal{N}(K)}\left|\sigma_{K, L}\right|\left(v_{K, L}^{n}+v_{L, K}^{n}\right) \sum_{L \in \mathcal{N}(K)}\left|\sigma_{K, L}\right|\left(v_{K, L}^{n}+v_{L, K}^{n}\right)\{\zeta A+(1-\zeta)(-B)\}^{2} \\
& \leqslant \sum_{L \in \mathcal{N}(K)}\left|\sigma_{K, L}\right|\left(v_{K, L}^{n}+v_{L, K}^{n}\right) \sum_{L \in \mathcal{N}(K)}\left|\sigma_{K, L}\right|\left(v_{K, L}^{n}+v_{L, K}^{n}\right)\left\{\zeta A^{2}+(1-\zeta) B^{2}\right\} .
\end{aligned}
$$

Since $\left(v_{K, L}^{n}+v_{L, K}^{n}\right) \zeta=v_{K, L}^{n}$ and $\left(v_{K, L}^{n}+v_{L, K}^{n}\right)(1-\zeta)=v_{L, K}^{n}$, we get the following estimates

$$
\begin{array}{r}
\sum_{K \in \mathcal{T}} B_{1} \leqslant \sum_{K \in \mathcal{T}} \frac{k^{2}}{2|K|}\left(\sum_{L \in \mathcal{N}(K)}\left|\sigma_{K, L}\right|\left(v_{K, L}^{n}+v_{L, K}^{n}\right)\right) \times E\left[\sum _ { L \in \mathcal { N } ( K ) } | \sigma _ { K , L } | \left\{v_{K, L}^{n}\left(F\left(u_{K}^{n}, u_{L}^{n}\right)-f\left(u_{K}^{n}\right)\right)^{2}\right.\right. \\
\left.\left.+v_{L, K}^{n}\left(F\left(u_{L}^{n}, u_{K}^{n}\right)-f\left(u_{K}^{n}\right)\right)^{2}\right\}\right] .
\end{array}
$$

Using the fact that

$$
\sum_{L \in \mathcal{N}(K)}\left|\sigma_{K, L}\right|\left(v_{K, L}^{n}+v_{L, K}^{n}\right) \leqslant V|\partial K|
$$

which implies thanks to the mesh properties (4) and the CFL Condition 10 that

$$
\frac{k}{|K|} \sum_{L \in \mathcal{N}(K)}\left|\sigma_{K, L}\right|\left(v_{K, L}^{n}+v_{L, K}^{n}\right) \leqslant k V \frac{|\partial K|}{|K|} \leqslant \frac{\bar{\alpha}^{2} h}{V\left(F_{1}+F_{2}\right)} V \frac{1}{\bar{\alpha}^{2} h}=\frac{1}{F_{1}+F_{2}},
$$

one finally gets by reordering the summation in 15

$$
\begin{aligned}
\sum_{K \in \mathcal{T}} B_{1} \leqslant \sum_{(K, L) \in \mathfrak{T}_{n}} \frac{k\left|\sigma_{K, L}\right|}{2\left(F_{1}+F_{2}\right)} E[ & v_{K, L}^{n}\left\{\left(F\left(u_{K}^{n}, u_{L}^{n}\right)-f\left(u_{K}^{n}\right)\right)^{2}+\left(F\left(u_{K}^{n}, u_{L}^{n}\right)-f\left(u_{L}^{n}\right)\right)^{2}\right\} \\
& \left.+v_{L, K}^{n}\left\{\left(f\left(u_{K}^{n}\right)-F\left(u_{L}^{n}, u_{K}^{n}\right)\right)^{2}+\left(f\left(u_{L}^{n}\right)-F\left(u_{L}^{n}, u_{K}^{n}\right)\right)^{2}\right\}\right] .
\end{aligned}
$$

In this way, using 14 and 18 , since

$$
\sum_{K \in \mathcal{T}} \frac{|K|}{2} E\left[\left(u_{K}^{n+1}\right)^{2}\right]=\sum_{K \in \mathcal{T}}\left(B_{1}-B_{2}+D+\frac{|K|}{2} E\left[\left(u_{K}^{n}\right)^{2}\right]\right)
$$

one gets

$$
\begin{aligned}
\sum_{K \in \mathcal{T}} \frac{|K|}{2} E\left[\left(u_{K}^{n+1}\right)^{2}\right] & \leqslant \sum_{K \in \mathcal{T}} \frac{k|K|}{2} E\left[g\left(u_{K}^{n}\right)^{2}\right]+\sum_{K \in \mathcal{T}} \frac{|K|}{2} E\left[\left(u_{K}^{n}\right)^{2}\right] \\
& \leqslant \sum_{K \in \mathcal{T}} \frac{|K|}{2}\left(1+k C_{g}^{2}\right) E\left[\left(u_{K}^{n}\right)^{2}\right] .
\end{aligned}
$$


In this way, using $\left(P_{n}\right)$ we get

$$
\begin{aligned}
\sum_{K \in \mathcal{T}}|K| E\left[\left(u_{K}^{n+1}\right)^{2}\right] & \leqslant \sum_{K \in \mathcal{T}}|K|\left(1+k C_{g}^{2}\right) E\left[\left(u_{K}^{n}\right)^{2}\right] \\
& \leqslant\left(1+k C_{g}^{2}\right)^{n+1}\left\|u_{0}\right\|_{L^{2}\left(\mathbb{R}^{d}\right)}^{2}
\end{aligned}
$$

We deduce that $\left(P_{n+1}\right)$ holds, and we conclude by induction that

$$
\left\|u_{\mathcal{T}, k}\right\|_{L^{\infty}\left(0, T ; L^{2}\left(\Omega \times \mathbb{R}^{d}\right)\right)} \leqslant e^{C_{g}^{2} T / 2}\left\|u_{0}\right\|_{L^{2}\left(\mathbb{R}^{d}\right)} .
$$

This gives the $L_{t}^{\infty} L_{\omega, x}^{2}$ stability of the approximate solution. As a consequence, we have

$$
\begin{aligned}
\left\|u_{\mathcal{T}, k}\right\|_{L^{2}(\Omega \times Q)}^{2} & =\sum_{n=0}^{N-1} \sum_{K \in \mathcal{T}} k|K| E\left[\left(u_{K}^{n}\right)^{2}\right] \\
& \leqslant T e^{C_{g}^{2} T}\left\|u_{0}\right\|_{L^{2}\left(\mathbb{R}^{d}\right)}^{2} .
\end{aligned}
$$

\subsection{Weak BV estimate}

Proposition 2 (Weak $\boldsymbol{B} \boldsymbol{V}$ estimate) Let $\mathcal{T}$ be an admissible mesh in the sense of Definition $3 . T>0$, $N \in \mathbb{N}^{\star}$ and let $k=\frac{T}{N} \in \mathbb{R}_{+}^{*}$ satisfying the CFL Condition

$$
k \leqslant(1-\xi) \frac{\bar{\alpha}^{2} h}{V\left(F_{1}+F_{2}\right)},
$$

for some $\xi \in(0,1)$.

Let $\left\{u_{K}^{n}, K \in \mathcal{T}, n \in\{0, \ldots, N-1\}\right\}$ be given by the finite volume scheme (6).

We have then the two following bounds:

1. There exists $C_{1} \in \mathbb{R}_{+}^{*}$, only depending on $T, u_{0}, \xi, F_{1}, F_{2}$ and $C_{g}$ such that

$$
\sum_{n=0}^{N-1} k \sum_{K \in \mathcal{T}} \sum_{L \in \mathcal{N}(K)}\left|\sigma_{K, L}\right| E\left[v_{K, L}^{n}\left\{F\left(u_{K}^{n}, u_{L}^{n}\right)-f\left(u_{K}^{n}\right)\right\}^{2}+v_{L, K}^{n}\left\{F\left(u_{L}^{n}, u_{K}^{n}\right)-f\left(u_{K}^{n}\right)\right\}^{2}\right] \leqslant C_{1} .
$$

2. Let $R>0$ be such that $h<R$. Then, there exists $C_{2} \in \mathbb{R}_{+}^{*}$, only depending on $R, d, T, \bar{\alpha}, u_{0}, \xi, F_{1}, F_{2}$ and $C_{g}$ such that

$$
\begin{aligned}
\sum_{n=0}^{N-1} k \sum_{(K, L) \in \mathfrak{T}_{n}^{R}}\left|\sigma_{K, L}\right| E[ & v_{K, L}^{n}\left\{\max _{u_{L}^{n} \leqslant c \leqslant d \leqslant u_{K}^{n}}(F(d, c)-f(d))+\max _{u_{L}^{n} \leqslant c \leqslant d \leqslant u_{K}^{n}}(F(d, c)-f(c))\right\} \\
& \left.+v_{L, K}^{n}\left\{\max _{u_{L}^{n} \leqslant c \leqslant d \leqslant u_{K}^{n}}(f(d)-F(c, d))+\max _{u_{L}^{n} \leqslant c \leqslant d \leqslant u_{K}^{n}}(f(c)-F(c, d))\right\}\right] \leqslant C_{2} h^{-1 / 2},
\end{aligned}
$$

where

$$
\mathcal{T}_{R}=\{K \in \mathcal{T} \text { such that } K \subset B(0, R)\} \text { and } \mathfrak{T}_{n}^{R}=\left\{(K, L) \in \mathcal{T}_{R}^{2} \text { such that } L \in \mathcal{N}(K) \text { and } u_{K}^{n}>u_{L}^{n}\right\} .
$$

Proof. Similarly to the proof of Proposition 1, multiplying the numerical scheme by $k u_{K}^{n}$, taking the expectation, summing over $K \in \mathcal{T}$ and using the independence properties of the Brownian motion yields

$$
A+B=0
$$

where

$$
\begin{aligned}
A & =A_{1}+A_{2} \\
A_{1} & =-\frac{1}{2} \sum_{n=0}^{N-1} \sum_{K \in \mathcal{T}}\left\{k|K| E\left[\left(g\left(u_{K}^{n}\right)\right)^{2}\right]+\frac{k^{2}}{|K|} E\left[\left(\sum_{L \in \mathcal{N}(K)}\left|\sigma_{K, L}\right|\left\{v_{K, L}^{n}\left(F\left(u_{K}^{n}, u_{L}^{n}\right)-f\left(u_{K}^{n}\right)\right)-v_{L, K}^{n}\left(F\left(u_{L}^{n}, u_{K}^{n}\right)-f\left(u_{K}^{n}\right)\right)\right\}\right)^{2}\right]\right\} \\
A_{2} & =\frac{1}{2} \sum_{K \in \mathcal{T}}|K| E\left[\left(u_{K}^{N}\right)^{2}-\left(u_{K}^{0}\right)^{2}\right] \\
\text { and } & \\
B & =\sum_{n=0}^{N-1} \sum_{K \in \mathcal{T}} \sum_{L \in \mathcal{N}(K)} k\left|\sigma_{K, L}\right| E\left[\left\{v_{K, L}^{n}\left(F\left(u_{K}^{n}, u_{L}^{n}\right)-f\left(u_{K}^{n}\right)\right)-v_{L, K}^{n}\left(F\left(u_{L}^{n}, u_{K}^{n}\right)-f\left(u_{K}^{n}\right)\right)\right\} u_{K}^{n}\right] .
\end{aligned}
$$


Similarly to (17), it follows from the CFL Condition (19) and the mesh properties (4) that

$$
\frac{k}{|K|} \sum_{L \in \mathcal{N}(K)}\left|\sigma_{K, L}\right|\left(v_{K, L}^{n}+v_{L, K}^{n}\right) \leqslant(1-\xi) \frac{1}{F_{1}+F_{2}} .
$$

Using 20 and Cauchy-Schwarz inequality, we deduce that

$$
\begin{aligned}
A_{1} \geqslant- & -\frac{1}{2} \sum_{n=0}^{N-1} k \sum_{K \in \mathcal{T}}|K| E\left[\left(g\left(u_{K}^{n}\right)\right)^{2}\right] \\
-\frac{(1-\xi)}{2} \sum_{n=0}^{N-1} k \sum_{(K, L) \in \mathfrak{T}_{n}} \frac{\left|\sigma_{K, L}\right|}{\left(F_{1}+F_{2}\right)} E & {\left[v_{K, L}^{n}\left\{\max _{u_{L}^{n} \leqslant c \leqslant d \leqslant u_{K}^{n}}(F(d, c)-f(d))^{2}+\max _{u_{L}^{n} \leqslant c \leqslant d \leqslant u_{K}^{n}}(F(d, c)-f(c))^{2}\right\}\right.} \\
& \left.+v_{L, K}^{n}\left\{\max _{u_{L}^{n} \leqslant c \leqslant d \leqslant u_{K}^{n}}(f(d)-F(c, d))^{2}+\max _{u_{L}^{n} \leqslant c \leqslant d \leqslant u_{K}^{n}}(f(c)-F(c, d))^{2}\right\}\right],
\end{aligned}
$$

where we recall that $\mathfrak{T}_{n}=\left\{(K, L) \in \mathcal{T}^{2}: L \in \mathcal{N}(K)\right.$ and $\left.u_{K}^{n}>u_{L}^{n}\right\}$.

Thus,

$A \geqslant-\frac{1}{2} T C_{g}^{2} e^{T C_{g}^{2}}\left\|u_{0}\right\|_{L^{2}\left(\mathbb{R}^{d}\right)}^{2}-\frac{1}{2}\left\|u_{0}\right\|_{L^{2}\left(\mathbb{R}^{d}\right)}^{2}$

$$
\begin{aligned}
-\frac{(1-\xi)}{2\left(F_{1}+F_{2}\right)} \sum_{n=0}^{N-1} k \sum_{(K, L) \in \mathfrak{T}_{n}}\left|\sigma_{K, L}\right| E[ & v_{K, L}^{n}\left\{\max _{u_{L}^{n} \leqslant c \leqslant d \leqslant u_{K}^{n}}(F(d, c)-f(d))^{2}+\max _{u_{L}^{n} \leqslant c \leqslant d \leqslant u_{K}^{n}}(F(d, c)-f(c))^{2}\right\} \\
& \left.+v_{L, K}^{n}\left\{\max _{u_{L}^{n} \leqslant c \leqslant d \leqslant u_{K}^{n}}(f(d)-F(c, d))^{2}+\max _{u_{L}^{n} \leqslant c \leqslant d \leqslant u_{K}^{n}}(f(c)-F(c, d))^{2}\right\}\right] .
\end{aligned}
$$

We now study the term

$$
B=\sum_{n=0}^{N-1} k \sum_{K \in \mathcal{T}} \sum_{L \in \mathcal{N}(K)}\left|\sigma_{K, L}\right| E\left[\left\{v_{K, L}^{n}\left(F\left(u_{K}^{n}, u_{L}^{n}\right)-f\left(u_{K}^{n}\right)\right)-v_{L, K}^{n}\left(F\left(u_{L}^{n}, u_{K}^{n}\right)-f\left(u_{K}^{n}\right)\right)\right\} u_{K}^{n}\right] .
$$

Reordering the terms and using the result obtained in the proof of Proposition 1 (the fact that $B_{3}=B_{4}$ ), we get:

$$
\begin{gathered}
k \sum_{(K, L) \in \mathfrak{T}_{n}}\left|\sigma_{K, L}\right| E\left[v_{K, L}^{n}\left\{u_{K}^{n}\left(F\left(u_{K}^{n}, u_{L}^{n}\right)-f\left(u_{K}^{n}\right)\right)-u_{L}^{n}\left(F\left(u_{K}^{n}, u_{L}^{n}\right)-f\left(u_{L}^{n}\right)\right)\right\}\right. \\
\left.\quad-v_{L, K}^{n}\left\{u_{K}^{n}\left(F\left(u_{L}^{n}, u_{K}^{n}\right)-f\left(u_{K}^{n}\right)\right)-u_{L}^{n}\left(F\left(u_{L}^{n}, u_{K}^{n}\right)-f\left(u_{L}^{n}\right)\right)\right\}\right] \\
=k \sum_{(K, L) \in \mathfrak{T}_{n}}\left|\sigma_{K, L}\right| E\left[\left\{v_{K, L}^{n} \int_{u_{K}^{n}}^{u_{L}^{n}} f(s)-F\left(u_{K}^{n}, u_{L}^{n}\right) d s+v_{L, K}^{n} \int_{u_{L}^{n}}^{u_{K}^{n}} f(s)-F\left(u_{L}^{n}, u_{K}^{n}\right) d s\right\}\right]
\end{gathered}
$$

Taking the sum over $n$, one can then rewrite $B$ in the following way

$$
\begin{aligned}
& B=\sum_{n=0}^{N-1} k \sum_{(K, L) \in \mathfrak{T}_{n}}\left|\sigma_{K, L}\right| E[ v_{K, L}^{n}\left\{u_{K}^{n}\left(F\left(u_{K}^{n}, u_{L}^{n}\right)-f\left(u_{K}^{n}\right)\right)-u_{L}^{n}\left(F\left(u_{K}^{n}, u_{L}^{n}\right)-f\left(u_{L}^{n}\right)\right)\right\} \\
&\left.-v_{L, K}^{n}\left\{u_{K}^{n}\left(F\left(u_{L}^{n}, u_{K}^{n}\right)-f\left(u_{K}^{n}\right)\right)-u_{L}^{n}\left(F\left(u_{L}^{n}, u_{K}^{n}\right)-f\left(u_{L}^{n}\right)\right)\right\}\right] \\
&=\sum_{n=0}^{N-1} k \sum_{(K, L) \in \mathfrak{T}_{n}}\left|\sigma_{K, L}\right| E\left[\left\{v_{K, L}^{n} \int_{u_{K}^{n}}^{u_{L}^{n}} f(s)-F\left(u_{K}^{n}, u_{L}^{n}\right) d s+v_{L, K}^{n} \int_{u_{L}^{n}}^{u_{K}^{n}} f(s)-F\left(u_{L}^{n}, u_{K}^{n}\right) d s\right\}\right]
\end{aligned}
$$

Let us now turn to an estimate of $B$.

For this purpose, let $a, b \in \mathbb{R}$ and define $\mathcal{C}(a, b)=\left\{(c, d) \in[\min (a, b), \max (a, b)]^{2}:(d-c)(b-a) \geqslant 0\right\}$. Thanks to the monotonicity of $F$, the following inequality holds for any $(c, d) \in \mathcal{C}(a, b)$ :

$$
\int_{a}^{b} f(s)-F(a, b) d s \geqslant \int_{c}^{d} f(s)-F(a, b) d s \geqslant \int_{c}^{d} f(s)-F(c, d) d s .
$$

We now use again Lemma $1 \mathrm{p} 7$ and deduce that for all $(c, d) \in \mathcal{C}(a, b)$ : and

$$
\int_{a}^{b} f(s)-F(a, b) d s \geqslant \int_{c}^{d} f(s)-F(c, d) d s \geqslant \int_{c}^{d} F(c, s)-F(c, d) d s \geqslant \frac{1}{2 F_{2}}(f(c)-F(c, d))^{2}
$$

$$
\int_{a}^{b} f(s)-F(a, b) d s \geqslant \int_{c}^{d} f(s)-F(c, d) d s \geqslant \int_{c}^{d} F(s, d)-F(c, d) d s \geqslant \frac{1}{2 F_{1}}(f(d)-F(c, d))^{2} .
$$


Multiplying 21 (respectively 22 ) by $\frac{F_{2}}{F_{1}+F_{2}}$ (respectively by $\left.\frac{F_{1}}{F_{1}+F_{2}}\right)$, taking the maximum for $(c, d) \epsilon$ $\mathcal{C}(a, b)$ and adding the two inequalities yields:

$$
\int_{a}^{b} f(s)-F(a, b) d s \geqslant \frac{1}{2\left(F_{1}+F_{2}\right)}\left[\max _{(c, d) \in \mathcal{C}(a, b)}(f(c)-F(c, d))^{2}+\max _{(c, d) \in \mathcal{C}(a, b)}(f(d)-F(c, d))^{2}\right] .
$$

Taking the sum over $n$, we can deduce from this last inequality that

$$
\begin{aligned}
B \geqslant \sum_{n=0}^{N-1} k \sum_{(K, L) \in \mathfrak{T}_{n}} \frac{\left|\sigma_{K, L}\right|}{2\left(F_{1}+F_{2}\right)} E & {\left[v_{K, L}^{n}\left\{\max _{u_{L}^{n} \leqslant c \leqslant d \leqslant u_{K}^{n}}(F(d, c)-f(d))^{2}+\max _{u_{L}^{n} \leqslant c \leqslant d \leqslant u_{K}^{n}}(F(d, c)-f(c))^{2}\right\}\right.} \\
& \left.+v_{L, K}^{n}\left\{\max _{u_{L}^{n} \leqslant c \leqslant d \leqslant u_{K}^{n}}(f(d)-F(c, d))^{2}+\max _{u_{L}^{n} \leqslant c \leqslant d \leqslant u_{K}^{n}}(f(c)-F(c, d))^{2}\right\}\right] .
\end{aligned}
$$

Therefore, since $A+B=0$, by denoting $\tilde{C}=\left(1+T C_{g}^{2} e^{T C_{g}^{2}}\right)\left\|u_{0}\right\|_{L^{2}\left(\mathbb{R}^{d}\right)}^{2}$

$$
\begin{aligned}
\frac{1}{2} \tilde{C} \geqslant \frac{\xi}{2\left(F_{1}+F_{2}\right)} \sum_{n=0}^{N-1} k \sum_{(K, L) \in \mathfrak{T}_{n}}\left|\sigma_{K, L}\right| E\left[v_{K, L}^{n}\left\{\max _{u_{L}^{n} \leqslant c \leqslant d \leqslant u_{K}^{n}}(F(d, c)-f(d))^{2}+\max _{u_{L}^{n} \leqslant c \leqslant d \leqslant u_{K}^{n}}(F(d, c)-f(c))^{2}\right\}\right. \\
\left.+v_{L, K}^{n}\left\{\max _{u_{L}^{n} \leqslant s \leqslant d \leqslant u_{K}^{n}}(f(d)-F(c, d))^{2}+\max _{u_{L}^{n} \leqslant c \leqslant d \leqslant u_{K}^{n}}(f(c)-F(c, d))^{2}\right\}\right],
\end{aligned}
$$

which, in turn, gives the existence of $C_{1} \in \mathbb{R}_{+}^{*}$, only depending on $T, C_{f}, C_{g}, \xi$ and $\left\|u_{0}\right\|_{L^{2}\left(\mathbb{R}^{d}\right)}$ such that

$$
\begin{aligned}
\sum_{n=0}^{N-1} k \sum_{(K, L) \in \mathfrak{T}_{n}}\left|\sigma_{K, L}\right| & {\left[v_{K, L}^{n}\left\{\max _{u_{L}^{n} \leqslant c \leqslant d \leqslant u_{K}^{n}}(F(d, c)-f(d))^{2}+\max _{u_{L}^{n} \leqslant c \leqslant d \leqslant u_{K}^{n}}(F(d, c)-f(c))^{2}\right\}\right.} \\
+ & \left.v_{L, K}^{n}\left\{\max _{u_{L}^{n} \leqslant c \leqslant d \leqslant u_{K}^{n}}(f(d)-F(c, d))^{2}+\max _{u_{L}^{n} \leqslant c \leqslant d \leqslant u_{K}^{n}}(f(c)-F(c, d))^{2}\right\}\right] \leqslant C_{1} .
\end{aligned}
$$

Moreover by reordering the summation we have in particular

$$
\sum_{n=0}^{N-1} k \sum_{K \in \mathcal{T}} \sum_{\substack{\sigma \in \mathcal{E}_{K} \\ \sigma=\sigma_{K, L}}}|\sigma| E\left[v_{K, L}^{n}\left\{F\left(u_{K}^{n}, u_{L}^{n}\right)-f\left(u_{K}^{n}\right)\right\}^{2}+v_{L, K}^{n}\left\{F\left(u_{L}^{n}, u_{K}^{n}\right)-f\left(u_{K}^{n}\right)\right\}^{2}\right] \leqslant C_{1},
$$

which proves the first point of the proposition. Let us now turn to the second point of the proposition. Set $R>0$ be such that $h<R$ and define the sets

$$
\mathcal{T}_{R}=\{K \in \mathcal{T} \text { such that } K \subset B(0, R)\} \text { and } \mathfrak{T}_{n}^{R}=\left\{(K, L) \in \mathcal{T}_{R}^{2} \text { such that } L \in \mathcal{N}(K) \text { and } u_{K}^{n}>u_{L}^{n}\right\} .
$$

Now we aim to estimate

$$
\begin{aligned}
\left\{\sum_{n=0}^{N-1} k \sum_{(K, L) \in \mathfrak{T} R}\left|\sigma_{K, L}\right| E\right. & {\left[v_{K, L}^{n}\left\{\max _{u_{L}^{n} \leqslant c \leqslant d \leqslant u_{K}^{n}}(F(d, c)-f(d))+\max _{u_{L}^{n} \leqslant c \leqslant d \leqslant u_{K}^{n}}(F(d, c)-f(c))\right\}\right.} \\
& \left.\left.+v_{L, K}^{n}\left\{\max _{u_{L}^{n} \leqslant c \leqslant d \leqslant u_{K}^{n}}(f(d)-F(c, d))+\max _{u_{L}^{n} \leqslant c \leqslant d \leqslant u_{K}^{n}}(f(c)-F(c, d))\right\}\right]\right\}^{2} .
\end{aligned}
$$

Let us denote by

and

$$
T_{1}=\max _{u_{L}^{n} \leqslant c \leqslant d \leqslant u_{K}^{n}}(F(d, c)-f(d))+\max _{u_{L}^{n} \leqslant c \leqslant d \leqslant u_{K}^{n}}(F(d, c)-f(c))
$$

$$
T_{2}=\max _{u_{L}^{n} \leqslant c \leqslant d \leqslant u_{K}^{n}}(f(d)-F(c, d))+\max _{u_{L}^{n} \leqslant c \leqslant d \leqslant u_{K}^{n}}(f(c)-F(c, d))
$$

Using Cauchy-Schwarz inequality, one gets

$$
\begin{aligned}
& \left(\sum_{n=0}^{N-1} k \sum_{(K, L) \in \mathfrak{T}_{n}^{R}}\left|\sigma_{K, L}\right| E\left[v_{K, L}^{n} T_{1}+v_{L, K}^{n} T_{2}\right]\right)^{2} \\
\leqslant & \left(\sum_{n=0}^{N-1} k \sum_{(K, L) \in \mathfrak{T}_{n}^{R}}\left|\sigma_{K, L}\right|\left(v_{K, L}^{n}+v_{L, K}^{n}\right)\right) \times\left(\sum_{n=0}^{N-1} k \sum_{(K, L) \in \mathfrak{T}_{n}^{R}}\left|\sigma_{K, L}\right| E\left[\frac{\left(v_{K, L}^{n} T_{1}+v_{L, K}^{n} T_{2}\right)^{2}}{v_{K, L}^{n}+v_{L, K}^{n}}\right]\right) \\
\leqslant & \left(\sum_{n=0}^{N-1} k \sum_{(K, L) \in \mathfrak{T}_{n}^{R}}\left|\sigma_{K, L}\right|\left(v_{K, L}^{n}+v_{L, K}^{n}\right)\right) \times\left(\sum_{n=0}^{N-1} k \sum_{(K, L) \in \mathfrak{T}_{n}^{R}}\left|\sigma_{K, L}\right| E\left[v_{K, L}^{n} T_{1}^{2}+v_{L, K}^{n} T_{2}^{2}\right]\right),
\end{aligned}
$$


where we have used the convexity inequality $\left(\frac{v_{K, L}^{n} T_{1}+v_{L, K}^{n} T_{2}}{v_{K, L}^{n}+v_{L, K}^{n}}\right)^{2} \leq \frac{v_{K, L}^{n}}{v_{K, L}^{n}+v_{L, K}^{n}} T_{1}^{2}+\frac{v_{L, K}^{n}}{v_{K, L}^{n}+v_{L, K}^{n}} T_{2}^{2}$. Note that

$$
\sum_{n=0}^{N-1} k \sum_{(K, L) \in \mathfrak{T}_{n}^{R}}\left|\sigma_{K, L}\right|\left(v_{K, L}^{n}+v_{L, K}^{n}\right) \leqslant T \sum_{K \in \mathcal{T}_{R}}|\partial K| V \leqslant T \operatorname{Card}\left(\mathcal{T}_{R}\right) \max _{K \in \mathcal{T}_{R}}|\partial K| V
$$

and by deducing from the mesh properties that $\max _{K \in \mathcal{T}_{R}}|\partial K| \leqslant \frac{1}{\bar{\alpha}} h^{d-1}$ and the bound

$$
\operatorname{Card}\left(\mathcal{T}_{R}\right) \leqslant \frac{|B(0, R)|}{\min _{K \in \mathcal{T}_{R}}|K|} \leqslant \frac{|B(0, R)|}{\bar{\alpha} h^{d}}
$$

we have

$$
\sum_{n=0}^{N-1} k \sum_{(K, L) \in \mathfrak{T}_{n}^{R}}\left|\sigma_{K, L}\right|\left(v_{K, L}^{n}+v_{L, K}^{n}\right) \leqslant \frac{T V|B(0, R)|}{\bar{\alpha}^{2} h} .
$$

Finally, using (23) and (24), the fact that

$$
\begin{aligned}
& T_{1}^{2} \leqslant 2\left\{\max _{u_{L}^{n} \leqslant c \leqslant d \leqslant u_{K}^{n}}(F(d, c)-f(d))^{2}+\max _{u_{L}^{n} \leqslant c \leqslant d \leqslant u_{K}^{n}}(F(d, c)-f(c))^{2}\right\} \\
& T_{2}^{2} \leqslant 2\left\{\max _{u_{L}^{n} \leqslant c \leqslant d \leqslant u_{K}^{n}}(f(d)-F(c, d))^{2}+\max _{u_{L}^{n} \leqslant c \leqslant d \leqslant u_{K}^{n}}(f(c)-F(c, d))^{2}\right\}
\end{aligned}
$$

one finally gets

$$
\begin{aligned}
\left(\sum _ { n = 0 } ^ { N - 1 } k \sum _ { ( K , L ) \in \mathfrak { T } _ { n } ^ { R } } | \sigma _ { K , L } | E \left[v_{K, L}^{n}\left\{\max _{u_{L}^{n} \leqslant c \leqslant d \leqslant u_{K}^{n}}(F(d, c)-f(d))+\max _{u_{L}^{n} \leqslant c \leqslant d \leqslant u_{K}^{n}}(F(d, c)-f(c))\right\}\right.\right. \\
\left.\left.+v_{L, K}^{n}\left\{\max _{u_{L}^{n} \leqslant c \leqslant d \leqslant u_{K}^{n}}(f(d)-F(c, d))+\max _{u_{L}^{n} \leqslant c \leqslant d \leqslant u_{K}^{n}}(f(c)-F(c, d))\right\}\right]\right)^{2} \leqslant \frac{2 T C_{1}|B(0, R)| V}{\bar{\alpha}^{2} h},
\end{aligned}
$$

which concludes the proof of the second point of the proposition.

\subsection{Convergence of the finite volume approximate solution}

First of all, note that the a priori estimates stated in Proposition 1 only provide (up to a subsequence) weak convergences for $u_{\mathcal{T}, k}$. Moreover, due to the nonlinearity of $f$ and $g$, one needs compactness arguments to pass to the limit in the nonlinear terms and these arguments have to be compatible with the random variable. The concept of Young measures is appropriate here and the technique is based on the notion of narrow convergence of Young measures (or entropy processes), we refer to BALDER Bal00, but also to Eymard-GallouËt-Herbin EGH95.

In this way, taking a sequence of approximate finite volume solution, $u_{\mathcal{T}, k}$, it converges (up to a subsequence still denoted $u_{\mathcal{T}, k}$ ) in the sense of Young measures to an "entropy process" denoted by $\mathbf{u}$ which belongs to $L^{2}(\Omega \times Q \times(0,1))$. Precisely, given a Carathéodory function $\Psi: \Omega \times Q \times \mathbb{R} \rightarrow \mathbb{R}$ such that $\Psi\left(., u_{\mathcal{T}, k}\right)$ is uniformly integrable, one has:

$$
E\left[\int_{Q} \Psi\left(., u_{\mathcal{T}, k}\right) d x d t\right] \rightarrow E\left[\int_{Q} \int_{0}^{1} \Psi(., \mathbf{u}(., \alpha)) d \alpha d x d t\right] .
$$

A proof of this result can be found in BVW12, Section A.3.2. We recall that a function $\Psi: \Omega \times Q \times \mathbb{R} \rightarrow \mathbb{R}$ is a Carathéodory function if for almost any $(\omega, x, t) \in \Omega \times Q$ the function $\nu \mapsto \Psi(\omega, x, t, \nu)$ is continuous and for all $\nu \in \mathbb{R}$, the function $(\omega, x, t) \mapsto \Psi(\omega, x, t, \nu)$ is measurable. We also recall that a sequence $\left(\psi_{n}\right)_{n \geq 0}$ of functions $\psi_{n}: \Omega \times Q \rightarrow \mathbb{R}$ is said to be uniformly integrable (see BVW12, Section A.3.2.) if it satisfies the following properties:

. $\left(\psi_{n}\right)_{n \geq 0}$ is bounded in $L^{1}(\Omega \times Q)$.

- $\left(\psi_{n}\right)_{n \geq 0}$ is equi-integrable, that is to say that for any $\varepsilon>0$, there exists $\delta>0$ such that for any measurable set $A$ of $\Omega \times Q$ satisfying $\left(\mathcal{L}^{d+1} \otimes P\right)(A) \leqslant \delta$, we have for any $n \in \mathbb{N}$,

$$
\int_{A}\left|\psi_{n}(\omega, x, t)\right| d x d t d P \leqslant \varepsilon
$$

(where $\mathcal{L}^{d+1}$ is the $d+1$-dimensional Lebesgue measure). 
For any $\varepsilon>0$ there exists a measurable set $K_{\varepsilon}$ of $\Omega \times Q$ with $\left(\mathcal{L}^{d+1} \otimes P\right)\left(K_{\epsilon}\right)<\infty$ and such that for any $n \in \mathbb{N}$ we have

$$
\int_{K_{\varepsilon}^{c}}\left|\psi_{n}(\omega, x, t)\right| d x d t d P \leqslant \varepsilon
$$

We recall the following classical result. If $K$ is a subset of $Q$ with finite measure, a sequence of function bounded in $L^{p}(\Omega \times K)$ for some $p>1$ is uniformly integrable.

Remark 9 (On the measurability of $\mathbf{u}$ ) Since $u_{\mathcal{T}, k}$ is bounded in the Hilbert space $\mathcal{N}_{w}^{2}\left(0, T, L^{2}\left(\mathbb{R}^{d}\right)\right)$, by identification one shows that $u_{\mathcal{T}, k} \rightarrow \int_{0}^{1} \mathbf{u}(., \alpha) d \alpha$ weakly in $L^{2}(\Omega \times Q)$ so that $\int_{0}^{1} \mathbf{u}(., \alpha) d \alpha$ is a predictable process with values in $L^{2}\left(\mathbb{R}^{d}\right)$. An interesting point is the measurability of $\mathbf{u}$ with respect to all its variables $(\omega, x, t, \alpha)$. Revisiting the work of PAnov [Pan96] with the $\sigma$-field $\mathcal{P}_{T} \otimes \mathcal{L}\left(\mathbb{R}^{d}\right)$, one shows that $\mathbf{u}$ is measurable for the $\sigma$-field $\mathcal{P}_{T} \otimes \mathcal{L}\left(\mathbb{R}^{d} \times\right] 0,1[)$, thus $\mathbf{u} \in \mathcal{N}_{w}^{2}\left(0, T, L^{2}\left(\mathbb{R}^{d} \times\right] 0,1[)\right)$. See Appendix A.3.3 p.707 [BVW12].

Remark $10\left(L^{\infty}\left(0, T ; L^{2}\left(\Omega \times \mathbb{R}^{d} \times(0,1)\right)\right)\right)$ regularity of $\left.\mathbf{u}\right)$ Since the sequence of approximate solutions $u_{\mathcal{T}, k}$ is bounded in $L^{\infty}\left(0, T ; L^{2}\left(\Omega \times \mathbb{R}^{d}\right)\right)$ according to Proposition 1 , following [BVW12] Remark 2.4 p.667668 we show that $\mathbf{u} \in L^{\infty}\left(0, T ; L^{2}\left(\Omega \times \mathbb{R}^{d} \times(0,1)\right)\right)$.

Note that if one is able to show that $\mathbf{u}$ is a measure-valued entropy solution of Problem (1) in the sense of Definition 2 then, using our reduction result stated in Theorem 1, we will be able to conclude that all the sequence $u_{\mathcal{T}, k}$ converges in $L_{\text {loc }}^{1}(\Omega \times Q)$ to the unique stochastic entropy solution of $(1)$ in the sense of Definition 1. Since $\mathbf{u}$ satisfied the regularities required by Definition 2, it remains to show that $\mathbf{u}$ satisfies the following entropy inequalities:

$\forall \eta \in \mathcal{A}, \forall \varphi \in \mathcal{D}^{+}\left(\mathbb{R}^{d} \times[0, T)\right)$ and P-a.s. in $\Omega$

$$
\begin{aligned}
0 \leqslant & \int_{\mathbb{R}^{d}} \eta\left(u_{0}\right) \varphi(x, 0) d x+\int_{Q} \int_{0}^{1}\left\{\eta(\mathbf{u}(., \alpha)) \partial_{t} \varphi(x, t)+\Phi(\mathbf{u}(., \alpha)) \vec{v}(x, t) \cdot \nabla_{x} \varphi(x, t)\right\} d \alpha d x d t \\
& +\int_{0}^{T} \int_{\mathbb{R}^{d}} \int_{0}^{1} \eta^{\prime}(\mathbf{u}(., \alpha)) g(\mathbf{u}(., \alpha)) \varphi(x, t) d \alpha d x d W(t) \\
& +\frac{1}{2} \int_{Q} \int_{0}^{1} g^{2}(\mathbf{u}(., \alpha)) \eta^{\prime \prime}(\mathbf{u}(., \alpha)) \varphi(x, t) d \alpha d x d t .
\end{aligned}
$$

This is the aim of the next section.

\section{Convergence of the scheme}

In order to show the convergence of the finite volume scheme (6), we are going to use the following lemma, which states that any general monotone numerical flux can be split into the sum of a Godunov flux and a modified Lax-Friedrichs flux (also known as Rusanov flux). More precisely, we have the following result, whose proof can be found in [CH00]. We give here a simple proof for the sake of completness.

Lemma 2 Any monotone flux F (i.e. any numerical flux satisfying Definition 4) can be written as a convex combination of a Godunov flux and a modified Lax-Friedrichs flux as follows:

For any $a, b \in \mathbb{R}$ there exists $\theta(a, b) \in[0,1]$ such that

$$
F(a, b)=\theta(a, b) F^{G}(a, b)+(1-\theta(a, b)) F_{D}^{L F}(a, b),
$$

where $F^{G}$ is a Godunov flux (see (28) below for the definition) and $F_{D}^{L F}$ is a modified Lax-Friedrichs flux with parameter $D=\max \left(F_{1}, F_{2}\right)$ satisfying :

$$
F_{D}^{L F}(a, b)=\frac{f(a)+f(b)}{2}-D(b-a)
$$

Note that since $D$ is fixed independently from $a$ and $b, F^{L F}$ is indeed a flux-splitting type scheme.

Proof. We give the proof in the case $a<b$ (the case $a>b$ is similar). If $a<b$, one has $F^{G}(a, b)=f(c)$ where $c \in[a, b]$ is such that $f(c)=\min \{f(d), d \in[a, b]\}$. Then, thanks to the fact that $F(c, c)=f(c)$ and to the monotony properties of $F$, one has (since $a \leqslant c \leqslant b) F(a, b) \leqslant F(c, b) \leqslant F(c, c)=f(c)=F^{G}(a, b)$.

From the other hand, the second property of $F$ (in Definition 4 ) gives $F(a, b) \geqslant F(a, a)-F_{2}(b-a) \geqslant$ $f(a)-D(b-a)$ and $F(a, b) \geqslant F(b, b)-F_{1}(b-a) \geqslant f(b)-D(b-a)$.

Adding this two inequalities leads to $F(a, b) \geqslant \frac{1}{2}(f(a)+f(b))-D(b-a)=F_{D}^{L F}(a, b)$. This proves that $F(a, b)$ is a convex combination between $F^{G}(a, b)$ and $F_{D}^{L F}(a, b)$ and concludes the proof of Lemma 2.

Note that to show the convergence of the scheme, we will treat in a first step the case where $F$ is a Godunov numerical flux. More precisely, the fact that $F$ is a Godunov numerical flux will be only use to study the terms $\tilde{B}^{h, k}-\bar{B}^{h, k}$ and $\bar{B}^{h, k}-B_{1}^{h, k}$ (defined below) in the points I.2 p 16 and II.3 p. 20 of the proof of 
Proposition 4 In a second step, we will explain how to treat the general case, i.e when $F$ is a general monotone numerical flux, by using the decomposition of Lemma 2 to study the terms $\tilde{B}^{h, k}-\bar{B}^{h, k}$ and $\bar{B}^{h, k}-B_{1}^{h, k}$.

We propose in this section entropy inequalities satisfied by the finite volume approximate solution and aim to pass to the limit in these formulations in order to show the convergence of the scheme. For technical reason, one considers a time-continuous approximate solution constructed from $u_{\mathcal{T}, k}$ and denoted $\bar{u}_{\mathcal{T}, k}$ in the sequel.

\subsection{A time-continuous approximation}

Set $K \in \mathcal{T}, n \in\{0, \ldots, N-1\}$ and consider $\bar{u}_{K}^{n}(s)$ the time-continuous stochastic process defined on $\Omega \times$ $[n k,(n+1) k)$ from the discrete unknowns $u_{K}^{n}$ by :

$$
\begin{aligned}
\bar{u}_{K}^{n}(s) & =u_{K}^{n}-\int_{n k}^{s} \frac{1}{|K|} \sum_{L \in \mathcal{N}(K)}\left|\sigma_{K, L}\right|\left\{v_{K, L}^{n} F\left(u_{K}^{n}, u_{L}^{n}\right)-v_{L, K}^{n} F\left(u_{L}^{n}, u_{K}^{n}\right)\right\} d t+\int_{n k}^{s} g\left(u_{K}^{n}\right) d W(t) \\
& =u_{K}^{n}-\frac{s-n k}{|K|} \sum_{L \in \mathcal{N}(K)}\left|\sigma_{K, L}\right|\left\{v_{K, L}^{n} F\left(u_{K}^{n}, u_{L}^{n}\right)-v_{L, K}^{n} F\left(u_{L}^{n}, u_{K}^{n}\right)\right\}+\int_{n k}^{s} g\left(u_{K}^{n}\right) d W(t) .
\end{aligned}
$$

In this way, we have for almost all $\omega, \bar{u}_{K}^{n}(\omega, n k)=u_{K}^{n}$ and $\bar{u}_{K}^{n}(\omega,(n+1) k)=u_{K}^{n+1}$ and therefore we can now define a time-continuous approximate solution $\bar{u}_{\mathcal{T}, k}$ on $\Omega \times \mathbb{R}^{d} \times[0, T)$ by

$$
\bar{u}_{\mathcal{T}, k}(\omega, x, t)=\bar{u}_{K}^{n}(\omega, t), \omega \in \Omega, x \in K \text { and } t \in[n k,(n+1) k] .
$$

Using again the fact that $\sum_{L \in \mathcal{N}(K)}\left|\sigma_{K, L}\right|\left(v_{K, L}^{n}-v_{L, K}^{n}\right)=0$ we can rewrite for any $K \in \mathcal{T}$ and $n \in\{0, \ldots, N-1\}$ the time-continuous approximate solution $\bar{u}_{\mathcal{T}, k}$ on $\Omega \times K \times[n k,(n+1) k]$ in the following way:

$$
\begin{aligned}
\bar{u}_{K}^{n}(s)= & u_{K}^{n}-\frac{s-n k}{|K|} \sum_{L \in \mathcal{N}(K)}\left|\sigma_{K, L}\right|\left\{v_{K, L}^{n}\left(F\left(u_{K}^{n}, u_{L}^{n}\right)-f\left(u_{K}^{n}\right)\right)-v_{L, K}^{n}\left(F\left(u_{L}^{n}, u_{K}^{n}\right)-f\left(u_{K}^{n}\right)\right)\right\} \\
& +\int_{n k}^{s} g\left(u_{K}^{n}\right) d W(t)
\end{aligned}
$$

We now estimate the difference between the continuous approximation $\bar{u}_{\mathcal{T}, k}$ and the finite volume solution $u_{\mathcal{T}, k}$.

Proposition 3 Let $u_{0} \in L^{2}\left(\mathbb{R}^{d}\right)$ and $\mathcal{T}$ be an admissible mesh in the sense of Definition $3, N \in \mathbb{N}^{\star}$ and let $k=\frac{T}{N} \in \mathbb{R}_{+}^{*}$ satisfying the CFL Condition (19). Let $\bar{u}_{\mathcal{T}, k}$ be the time-continuous approximate solution defined by (26), and $u_{\mathcal{T}, k}$ be the finite volume approximate solution defined by (5) and (6). Then there exists $c \in \mathbb{R}_{+}^{\star}$ depending only on $T, C_{g}, F_{1}, F_{2}, \bar{\alpha}, V$ and $u_{0}$ such that

$$
\left\|u_{\mathcal{T}, k}-\bar{u}_{\mathcal{T}, k}\right\|_{L^{2}(\Omega \times Q)}^{2} \leqslant c(h+k) .
$$

Proof. Using the equivalent definition 27] of $\bar{u}_{\mathcal{T}, k}$,

$$
\begin{aligned}
\left\|u_{\mathcal{T}, k}-\bar{u}_{\mathcal{T}, k}\right\|_{L^{2}(\Omega \times Q)}^{2} & =\sum_{K \in \mathcal{T}} \sum_{n=0}^{N-1} \int_{n k}^{(n+1) k} \int_{K} E\left[\left(-g\left(u_{K}^{n}\right)\left(W(s)-W^{n}\right)\right.\right. \\
& \left.\left.+\frac{s-n k}{|K|} \sum_{L \in \mathcal{N}(K)}\left|\sigma_{K, L}\right|\left\{v_{K, L}^{n}\left(F\left(u_{K}^{n}, u_{L}^{n}\right)-f\left(u_{K}^{n}\right)\right)-v_{L, K}^{n}\left(F\left(u_{L}^{n}, u_{K}^{n}\right)-f\left(u_{K}^{n}\right)\right)\right\}\right)^{2}\right] d x d s \\
= & \sum_{K \in \mathcal{T}} \sum_{n=0}^{N-1} \int_{n k}^{(n+1) k}\left\{|K| E\left[\left(g\left(u_{K}^{n}\right)\left(W^{n}-W(s)\right)\right)^{2}\right]\right. \\
& \left.+|K| E\left[\left(\frac{s-n k}{|K|} \sum_{L \in \mathcal{N}(K)}\left|\sigma_{K, L}\right|\left\{v_{K, L}^{n}\left(F\left(u_{K}^{n}, u_{L}^{n}\right)-f\left(u_{K}^{n}\right)\right)-v_{L, K}^{n}\left(F\left(u_{L}^{n}, u_{K}^{n}\right)-f\left(u_{K}^{n}\right)\right)\right\}\right)^{2}\right]\right\} d s \\
\leqslant & \sum_{K \in \mathcal{T}} \sum_{n=0}^{N-1}|K| k^{2} C_{g}^{2} E\left[\left(u_{K}^{n}\right)^{2}\right] \\
& +\sum_{K \in \mathcal{T}} \sum_{n=0}^{N-1} k \frac{k^{2}|K|}{|K|^{2}} E\left[\left(\sum_{L \in \mathcal{N}(K)}\left|\sigma_{K, L}\right|\left\{v_{K, L}^{n}\left(F\left(u_{K}^{n}, u_{L}^{n}\right)-f\left(u_{K}^{n}\right)\right)-v_{L, K}^{n}\left(F\left(u_{L}^{n}, u_{K}^{n}\right)-f\left(u_{K}^{n}\right)\right)\right\}\right)^{2}\right]
\end{aligned}
$$

We use now Cauchy-Schwarz inequality, the assumptions on the mesh (4), the CFL Condition (10) and then the first estimate given in Proposition 2 (note that we can apply this Proposition since the more restrictive 
CFL Condition $(19)$ is fulfilled):

$$
\begin{aligned}
& \left\|u_{\mathcal{T}, k}-\bar{u}_{\mathcal{T}, k}\right\|_{L^{2}(\Omega \times Q)}^{2} \\
\leqslant & k C_{g}^{2}\left\|u_{\mathcal{T}, k}\right\|_{L^{2}(\Omega \times Q)}^{2} \\
& +\sum_{n=0}^{N-1} k \sum_{K \in \mathcal{T}} \sum_{L \in \mathcal{N}(K)} k^{2} V \frac{|\partial K|}{|K|}\left|\sigma_{K, L}\right| E\left[v_{K, L}^{n}\left(F\left(u_{K}^{n}, u_{L}^{n}\right)-f\left(u_{K}^{n}\right)\right)^{2}+v_{L, K}^{n}\left(F\left(u_{L}^{n}, u_{K}^{n}\right)-f\left(u_{K}^{n}\right)\right)^{2}\right] \\
\leqslant & k C_{g}^{2}\left\|u_{\mathcal{T}, k}\right\|_{L^{2}(\Omega \times Q)}^{2}+C_{1} \frac{\bar{\alpha}^{2} h}{V\left(F_{1}+F_{2}\right)^{2}}
\end{aligned}
$$

where we have used the constant $C_{1}$ given by Proposition 2

\subsection{Entropy inequalities for the approximate solution}

In this section, entropy inequalities satisfied by the approximate solution are introduced (Proposition 6, and will be used in the proof of convergence of the numerical scheme (Theorem 3). In order to obtain these entropy inequalities, some discrete entropy inequalities satisfied by the approximate solution are first derived in the following proposition. From now on, we assume that $F$ is the Godunov flux, namely defined by :

$$
F(a, b)= \begin{cases}\min _{s \in[a, b]} f(s) & \text { if } a \leqslant b \\ \max _{s \in[b, a]} f(s) & \text { if } a \geqslant b\end{cases}
$$

For all $(a, b) \in \mathbb{R}^{2}$ we will denote by $s(a, b) \in[\min (a, b), \max (a, b)]$ a real such that $F(a, b)=f(s(a, b))$. We define then the associated numerical entropy flux $G$ by $G(a, b)=\Phi(s(a, b))$ for any $a, b \in \mathbb{R}$. Note that for all $a \in \mathbb{R}, G(a, a)=\Phi(a)$.

Proposition 4 (Discrete entropy inequalities) Assume that hypotheses $H_{1}$ to $H_{6}$ hold and that $F$ is the Godunov flux defined by (28). Let $\mathcal{T}$ be an admissible mesh in the sense of Definition $3, N \in \mathbb{N}^{\star}$ and let $k=\frac{T}{N} \in \mathbb{R}_{+}^{*}$ be the time step. Then P-a.s in $\Omega$, for any $\eta \in \mathcal{A}$ and for any $\varphi \in \mathcal{D}^{+}\left(\mathbb{R}^{d} \times[0, T)\right)$ :

$$
\begin{aligned}
& -\sum_{n=0}^{N-1} \sum_{K \in \mathcal{T}_{R}} \int_{K}\left(\eta\left(u_{K}^{n+1}\right)-\eta\left(u_{K}^{n}\right)\right) \varphi(x, n k) d x \\
& +\sum_{n=0}^{N-1} \sum_{K \in \mathcal{T}_{R}} \int_{n k}^{(n+1) k} \int_{K} \Phi\left(u_{K}^{n}\right) \vec{v}(x, t) \cdot \nabla_{x} \varphi(x, n k) d x d t \\
& +\sum_{n=0}^{N-1} \sum_{K \in \mathcal{T}_{R}} \int_{n k}^{(n+1) k} \int_{K} \eta^{\prime}\left(u_{K}^{n}\right) g\left(u_{K}^{n}\right) \varphi(x, n k) d x d W(t) \\
& +\frac{1}{2} \sum_{n=0}^{N-1} \sum_{K \in \mathcal{T}_{R}} \int_{n k}^{(n+1) k} \int_{K} \eta^{\prime \prime}\left(u_{K}^{n}\right) g^{2}\left(u_{K}^{n}\right) \varphi(x, n k) d x d t \\
& \geqslant R^{h, k}
\end{aligned}
$$

where for any P-measurable set $A, E\left[\mathbb{1}_{A} R^{h, k}\right] \rightarrow 0$ as $\left(h, \frac{k}{h}\right) \rightarrow(0,0)$.

Proof. In order to prove this proposition, we are going to show firstly that inequality $(29)$ holds for a certain $R^{h, k}$ and in a second time, we will prove that for any P-measurable set $A, E\left[\mathbb{1}_{A} R^{h, k}\right] \rightarrow 0$ as $h \rightarrow 0$. We will in particular use some technics from [EGH00] and [CH00] and adapt these technics to our case. Let $T>0, u_{0} \in L^{2}\left(\mathbb{R}^{d}\right), \mathcal{T}$ be an admissible mesh in the sense of Definition $3 \quad N \in \mathbb{N}^{\star}$ and $k=\frac{T}{N} \in \mathbb{R}_{+}^{*}$. We assume that $k / h \rightarrow 0$ as $h \rightarrow 0$, in this way we can suppose that the CFL Condition

$$
k \leqslant \frac{(1-\xi) \bar{\alpha}^{2} h}{\left(F_{1}+F_{2}\right) V}
$$

holds for some $\xi \in(0,1)$. In this manner, the estimates given by Proposition 1 and Proposition 2 hold. Consider $\eta \in \mathcal{A}$ and $\varphi \in \mathcal{D}^{+}\left(\mathbb{R}^{d} \times[0, T)\right)$, thus there exists $R>h$ such that supp $\varphi \subset B(0, R-h) \times[0, T)$. We also define $\mathcal{T}_{R}=\{K \in \mathcal{T}$ such that $K \subset B(0, R)\}$ and $\mathfrak{T}_{n}^{R}=\left\{(K, L) \in \mathcal{T}_{R}^{2}\right.$ such that $L \in \mathcal{N}(K)$ and $\left.u_{K}^{n}>u_{L}^{n}\right\}$.

STEP I: Existence of $R_{h, k}$ 
The application of Itô's formula to the process $\bar{u}_{K}^{n}$ defined by Equation 25 for some $K \in \mathcal{T}$ and the function $v \in \mathbb{R} \mapsto \eta(v) \in \mathbb{R}$ on the interval $[n k,(n+1) k]$ yields P-a.s in $\Omega$

$$
\begin{aligned}
\eta\left(\bar{u}_{K}^{n}((n+1) k)\right)= & \eta\left(\bar{u}_{K}^{n}(n k)\right)-\frac{1}{|K|} \int_{n k}^{(n+1) k} \eta^{\prime}\left(\bar{u}_{\mathcal{T}, k}(t)\right) \sum_{L \in \mathcal{N}(K)}\left|\sigma_{K, L}\right|\left\{v_{K, L}^{n} F\left(u_{K}^{n}, u_{L}^{n}\right)-v_{L, K}^{n} F\left(u_{L}^{n}, u_{K}^{n}\right)\right\} d t \\
& +\int_{n k}^{(n+1) k} \eta^{\prime}\left(\bar{u}_{\mathcal{T}, k}(t)\right) g\left(u_{K}^{n}\right) d W(t)+\frac{1}{2} \int_{n k}^{(n+1) k} \eta^{\prime \prime}\left(\bar{u}_{\mathcal{T}, k}(t)\right) g^{2}\left(u_{K}^{n}\right) d t .
\end{aligned}
$$

Let us multiply Equation 30 by $|K| \varphi_{K}^{n}$, where $\varphi_{K}^{n}=\frac{1}{|K|} \int_{K} \varphi(x, n k) d x$, and sum for all $K \in \mathcal{T}_{R}$ and $n \in\{0, \ldots, N-1\}$. One gets $\mathrm{P}$-a.s in $\Omega$

$$
\begin{aligned}
\sum_{n=0}^{N-1} \sum_{K \in \mathcal{T}_{R}}\left[\eta\left(u_{K}^{n+1}\right)-\eta\left(u_{K}^{n}\right)\right]|K| \varphi_{K}^{n}= & -\sum_{n=0}^{N-1} \sum_{K \in \mathcal{T}_{R}} \int_{n k}^{(n+1) k} \eta^{\prime}\left(\bar{u}_{\mathcal{T}, k}(t)\right) \sum_{L \in \mathcal{N}(K)}\left|\sigma_{K, L}\right|\left\{v_{K, L}^{n} F\left(u_{K}^{n}, u_{L}^{n}\right)-v_{L, K}^{n} F\left(u_{L}^{n}, u_{K}^{n}\right)\right\} d t \varphi_{K}^{n} \\
& +\sum_{n=0}^{N-1} \sum_{K \in \mathcal{T}_{R}} \int_{n k}^{(n+1) k} \eta^{\prime}\left(\bar{u}_{\mathcal{T}, k}(t)\right) g\left(u_{K}^{n}\right) d W(t)|K| \varphi_{K}^{n} \\
& +\frac{1}{2} \sum_{n=0}^{N-1} \sum_{K \in \mathcal{T}_{R}} \int_{n k}^{(n+1) k} \eta^{\prime \prime}\left(\bar{u}_{\mathcal{T}, k}(t)\right) g^{2}\left(u_{K}^{n}\right) d t|K| \varphi_{K}^{n} .
\end{aligned}
$$

This can be written as $A^{h, k}=-B^{h, k}+C^{h, k}+D^{h, k}$, where

$$
\begin{aligned}
A^{h, k} & =\sum_{n=0}^{N-1} \sum_{K \in \mathcal{T}_{R}}\left[\eta\left(u_{K}^{n+1}\right)-\eta\left(u_{K}^{n}\right)\right]|K| \varphi_{K}^{n} \\
B^{h, k} & =\sum_{n=0}^{N-1} \sum_{K \in \mathcal{T}_{R}} \int_{n k}^{(n+1) k} \eta^{\prime}\left(\bar{u}_{\mathcal{T}, k}(t)\right) \sum_{L \in \mathcal{N}(K)}\left|\sigma_{K, L}\right|\left\{v_{K, L}^{n} F\left(u_{K}^{n}, u_{L}^{n}\right)-v_{L, K}^{n} F\left(u_{L}^{n}, u_{K}^{n}\right)\right\} d t \varphi_{K}^{n} \\
C^{h, k} & =\sum_{n=0}^{N-1} \sum_{K \in \mathcal{T}_{R}} \int_{n k}^{(n+1) k} \eta^{\prime}\left(\bar{u}_{\mathcal{T}, k}(t)\right) g\left(u_{K}^{n}\right) d W(t)|K| \varphi_{K}^{n} \\
D^{h, k} & =\frac{1}{2} \sum_{n=0}^{N-1} \sum_{K \in \mathcal{T}_{R}} \int_{n k}^{(n+1) k} \eta^{\prime \prime}\left(\bar{u}_{\mathcal{T}, k}(t)\right) g^{2}\left(u_{K}^{n}\right) d t|K| \varphi_{K}^{n} .
\end{aligned}
$$

Let us analyze separately these terms.

I.1 Study of $A^{h, k}$ : we note that $-A^{h, k}$ is equal to the first left hand side term of inequality 29.

I.2 Study of $B^{h, k}$ : we decompose

$B^{h, k}=\sum_{n=0}^{N-1} \sum_{K \in \mathcal{T}_{R}} \int_{n k}^{(n+1) k} \frac{1}{|K|} \int_{K} \eta^{\prime}\left(\bar{u}_{\mathcal{T}, k}(t)\right) \sum_{L \in \mathcal{N}(K)}\left|\sigma_{K, L}\right|\left\{v_{K, L}^{n} F\left(u_{K}^{n}, u_{L}^{n}\right)-v_{L, K}^{n} F\left(u_{L}^{n}, u_{K}^{n}\right)\right\} \varphi(x, n k) d x d t$

in the following way

$$
B^{h, k}=B^{h, k}-\tilde{B}^{h, k}+\tilde{B}^{h, k}-\bar{B}^{h, k}+\bar{B}^{h, k}-B_{1}^{h, k}+B_{1}^{h, k},
$$

where

$$
\begin{aligned}
\tilde{B}^{h, k} & =\sum_{n=0}^{N-1} \sum_{K \in \mathcal{T}_{R}} \int_{n k}^{(n+1) k} \frac{1}{|K|} \int_{K} \eta^{\prime}\left(u_{K}^{n}\right) \sum_{L \in \mathcal{N}(K)}\left|\sigma_{K, L}\right|\left\{v_{K, L}^{n} F\left(u_{K}^{n}, u_{L}^{n}\right)-v_{L, K}^{n} F\left(u_{L}^{n}, u_{K}^{n}\right)\right\} \varphi(x, n k) d x d t \\
\bar{B}^{h, k} & =\sum_{n=0}^{N-1} \sum_{K \in \mathcal{T}_{R}} \int_{n k}^{(n+1) k} \frac{1}{|K|} \int_{K} \sum_{L \in \mathcal{N}(K)}\left|\sigma_{K, L}\right|\left\{v_{K, L}^{n} G\left(u_{K}^{n}, u_{L}^{n}\right)-v_{L, K}^{n} G\left(u_{L}^{n}, u_{K}^{n}\right)\right\} \varphi(x, n k) d x d t \\
B_{1}^{h, k} & =-\sum_{n=0}^{N-1} \sum_{K \in \mathcal{T}_{R}} \int_{n k}^{(n+1) k} \int_{K} \Phi\left(u_{K}^{n}\right) \vec{v}(x, t) . \nabla_{x} \varphi(x, n k) d x d t .
\end{aligned}
$$

We show that $\tilde{B}^{h, k}-\bar{B}^{h, k} \geqslant 0$ almost surely.

First we notice that by 8 we have

$$
\sum_{L \in \mathcal{N}(K)}\left|\sigma_{K, L}\right|\left(v_{K, L}^{n}-v_{L, K}^{n}\right) \Phi\left(u_{K}^{n}\right)=\sum_{L \in \mathcal{N}(K)}\left|\sigma_{K, L}\right|\left(v_{K, L}^{n}-v_{L, K}^{n}\right) f\left(u_{K}^{n}\right)=0 .
$$


Recall that for any $K \in \mathcal{T}, F\left(u_{K}^{n}, u_{K}^{n}\right)=f\left(u_{K}^{n}\right)$ and $G\left(u_{K}^{n}, u_{K}^{n}\right)=\Phi\left(u_{K}^{n}\right)$ and that $\Phi$ is defined by $\Phi(a)=\int_{0}^{a} \eta^{\prime}(t) f^{\prime}(t) d t$ for $a \in \mathbb{R}$. Hence we can rewrite $\tilde{B}^{h, k}-\bar{B}^{h, k}$ in the following way :

$$
\begin{array}{r}
\tilde{B}^{h, k}-\bar{B}^{h, k}=\sum_{n=0}^{N-1} \sum_{K \in \mathcal{T}_{R}} \frac{k}{|K|} \sum_{L \in \mathcal{N}(K)}\left|\sigma_{K, L}\right|\left\{v_{K, L}^{n}\left[\eta^{\prime}\left(u_{K}^{n}\right)\left(F\left(u_{K}^{n}, u_{L}^{n}\right)-f\left(u_{K}^{n}\right)\right)-\left(G\left(u_{K}^{n}, u_{L}^{n}\right)-\Phi\left(u_{K}^{n}\right)\right)\right]\right. \\
\left.-v_{L, K}^{n}\left[\eta^{\prime}\left(u_{K}^{n}\right)\left(F\left(u_{L}^{n}, u_{K}^{n}\right)-f\left(u_{K}^{n}\right)\right)-\left(G\left(u_{L}^{n}, u_{K}^{n}\right)-\Phi\left(u_{K}^{n}\right)\right)\right]\right\} \int_{K} \varphi(x, n k) d x .
\end{array}
$$

Let $K, L \in \mathcal{T}$ and suppose that $u_{K}^{n}<u_{L}^{n}$ (the case $u_{L}^{n}<u_{K}^{n}$ is similar).

We first determine the sign of $\eta^{\prime}\left(u_{K}^{n}\right)\left(F\left(u_{K}^{n}, u_{L}^{n}\right)-f\left(u_{K}^{n}\right)\right)-\left(G\left(u_{K}^{n}, u_{L}^{n}\right)-\Phi\left(u_{K}^{n}\right)\right)$. Using the fact that we are in the particular case where $F$ is the Godunov numerical flux, we know that there exists $s\left(u_{K}^{n}, u_{L}^{n}\right) \in\left[u_{K}^{n}, u_{L}^{n}\right]$ such that $F\left(u_{K}^{n}, u_{L}^{n}\right)=f\left(s\left(u_{K}^{n}, u_{L}^{n}\right)\right)=\min _{t \in\left[u_{K}^{n}, u_{L}^{n}\right]} f(t)$. In this way

$$
\begin{aligned}
& \eta^{\prime}\left(u_{K}^{n}\right)\left(F\left(u_{K}^{n}, u_{L}^{n}\right)-f\left(u_{K}^{n}\right)\right)-\left(G\left(u_{K}^{n}, u_{L}^{n}\right)-\Phi\left(u_{K}^{n}\right)\right) \\
= & \eta^{\prime}\left(u_{K}^{n}\right)\left(f\left(s\left(u_{K}^{n}, u_{L}^{n}\right)\right)-f\left(u_{K}^{n}\right)\right)-\left(\Phi\left(s\left(u_{K}^{n}, u_{L}^{n}\right)\right)-\Phi\left(u_{K}^{n}\right)\right) \\
= & \int_{u_{K}^{n}}^{s\left(u_{K}^{n}, u_{L}^{n}\right)} f^{\prime}(t) \eta^{\prime}\left(u_{K}^{n}\right) d t-\int_{u_{K}^{n}}^{s\left(u_{K}^{n}, u_{L}^{n}\right)} f^{\prime}(t) \eta^{\prime}(t) d t \\
= & \int_{u_{K}^{n}}^{s\left(u_{K}^{n}, u_{L}^{n}\right)} f^{\prime}(t)\left(\eta^{\prime}\left(u_{K}^{n}\right)-\eta^{\prime}(t)\right) d t \\
= & \int_{u_{K}^{n}}^{s\left(u_{K}^{n}, u_{L}^{n}\right)} f(t) \eta^{\prime \prime}(t) d t+f\left(s\left(u_{K}^{n}, u_{L}^{n}\right)\right)\left\{\eta^{\prime}\left(u_{K}^{n}\right)-\eta^{\prime}\left(s\left(u_{K}^{n}, u_{L}^{n}\right)\right)\right\} \\
\geqslant & \int_{u_{K}^{n}}^{s\left(u_{K}^{n}, u_{L}^{n}\right)} f\left(s\left(u_{K}^{n}, u_{L}^{n}\right)\right) \eta^{\prime \prime}(t) d t+f\left(s\left(u_{K}^{n}, u_{L}^{n}\right)\right)\left\{\eta^{\prime}\left(u_{K}^{n}\right)-\eta^{\prime}\left(s\left(u_{K}^{n}, u_{L}^{n}\right)\right)\right\} \\
\geqslant & 0 .
\end{aligned}
$$

Using the same technics, we show that $\eta^{\prime}\left(u_{K}^{n}\right)\left(F\left(u_{L}^{n}, u_{K}^{n}\right)-f\left(u_{K}^{n}\right)\right)-\left(G\left(u_{L}^{n}, u_{K}^{n}\right)-\Phi\left(u_{K}^{n}\right)\right) \leqslant 0$. Indeed, since $u_{L}^{n}>u_{K}^{n}$, there exists $s\left(u_{L}^{n}, u_{K}^{n}\right) \in\left[u_{K}^{n}, u_{L}^{n}\right]$ such that $F\left(u_{L}^{n}, u_{K}^{n}\right)=f\left(s\left(u_{L}^{n}, u_{K}^{n}\right)\right)=\max _{t \in\left[u_{K}^{n}, u_{L}^{n}\right]} f(t)$ and we have

$$
\begin{aligned}
& \eta^{\prime}\left(u_{K}^{n}\right)\left(F\left(u_{L}^{n}, u_{K}^{n}\right)-f\left(u_{K}^{n}\right)\right)-\left(G\left(u_{L}^{n}, u_{K}^{n}\right)-\Phi\left(u_{K}^{n}\right)\right) \\
= & \eta^{\prime}\left(u_{K}^{n}\right)\left(f\left(s\left(u_{L}^{n}, u_{K}^{n}\right)-f\left(u_{K}^{n}\right)\right)-\left(\Phi\left(s\left(u_{K}^{n}, u_{L}^{n}\right)\right)-\Phi\left(u_{K}^{n}\right)\right)\right. \\
= & \int_{u_{K}^{n}}^{s\left(u_{L}^{n}, u_{K}^{n}\right)} f^{\prime}(t)\left(\eta^{\prime}\left(u_{K}^{n}\right)-\eta^{\prime}(t)\right) d t \\
= & \int_{u_{K}^{n}}^{s\left(u_{L}^{n}, u_{K}^{n}\right)} f(t) \eta^{\prime \prime}(t) d t+f\left(s\left(u_{L}^{n}, u_{K}^{n}\right)\right)\left\{\eta^{\prime}\left(u_{K}^{n}\right)-\eta^{\prime}\left(s\left(u_{L}^{n}, u_{K}^{n}\right)\right)\right\} \\
\leqslant & \int_{u_{K}^{n}}^{s\left(u_{L}^{n}, u_{K}^{n}\right)} f\left(s\left(u_{L}^{n}, u_{K}^{n}\right)\right) \eta^{\prime \prime}(t) d t+f\left(s\left(u_{L}^{n}, u_{K}^{n}\right)\right)\left\{\eta^{\prime}\left(u_{K}^{n}\right)-\eta^{\prime}\left(s\left(u_{L}^{n}, u_{K}^{n}\right)\right)\right\} \\
\leqslant & 0 .
\end{aligned}
$$

Finally we get

$$
\begin{gathered}
v_{K, L}^{n}\left[\eta^{\prime}\left(u_{K}^{n}\right)\left(F\left(u_{K}^{n}, u_{L}^{n}\right)-f\left(u_{K}^{n}\right)\right)-\left(G\left(u_{K}^{n}, u_{L}^{n}\right)-\Phi\left(u_{K}^{n}\right)\right)\right] \\
-v_{L, K}^{n}\left[\eta^{\prime}\left(u_{K}^{n}\right)\left(F\left(u_{L}^{n}, u_{K}^{n}\right)-f\left(u_{K}^{n}\right)\right)-\left(G\left(u_{L}^{n}, u_{K}^{n}\right)-\Phi\left(u_{K}^{n}\right)\right)\right] \geqslant 0 .
\end{gathered}
$$

We deduce that we have for almost all $\omega \in \Omega$,

$$
\tilde{B}^{h, k}-\bar{B}^{h, k} \geqslant 0,
$$

and thus

$$
B^{h, k} \geqslant B^{h, k}-\tilde{B}^{h, k}+\bar{B}^{h, k}-B_{1}^{h, k}+B_{1}^{h, k}
$$

I.3 Study of $C^{h, k}$ : we decompose $C^{h, k}$ in the following way

$$
C^{h, k}=C^{h, k}-\tilde{C}^{h, k}+\tilde{C}^{h, k},
$$

where

$$
\tilde{C}^{h, k}=\sum_{K \in \mathcal{T}_{R}} \sum_{n=0}^{N-1} \int_{K} \int_{n k}^{(n+1) k} \eta^{\prime}\left(u_{K}^{n}\right) g\left(u_{K}^{n}\right) \varphi(x, n k) d W(t) d x .
$$


I.4 Study of $D^{h, k}$ : we decompose $D^{h, k}$ in the following way

$$
D^{h, k}=D^{h, k}-\tilde{D}^{h, k}+\tilde{D}^{h, k}
$$

where

$$
\tilde{D}^{h, k}=\frac{1}{2} \sum_{n=0}^{N-1} \sum_{K \in \mathcal{T}_{R}} \int_{n k}^{(n+1) k} \int_{K} \eta^{\prime \prime}\left(u_{K}^{n}\right) g^{2}\left(u_{K}^{n}\right) \varphi(x, n k) d x d t
$$

Conclusion of STEP I:

Since P a.s in $\Omega, A^{h, k}=-B^{h, k}+C^{h, k}+D^{h, k}$, we get by using inequality 32

$$
\begin{aligned}
-A^{h, k}-B_{1}^{h, k}+\tilde{C}^{h, k}+\tilde{D}^{h, k} & =\left(B^{h, k}-\tilde{B}^{h, k}\right)+\left(\tilde{B}^{h, k}-\bar{B}^{h, k}\right)+\left(\bar{B}^{h, k}-B_{1}^{h, k}\right)+\left(\tilde{C}^{h, k}-C^{h, k}\right)+\left(\tilde{D}^{h, k}-D^{h, k}\right) \\
& \geqslant B^{h, k}-\tilde{B}^{h, k}+\bar{B}^{h, k}-B_{1}^{h, k}+\left(\tilde{C}^{h, k}-C^{h, k}\right)+\left(\tilde{D}^{h, k}-D^{h, k}\right) .
\end{aligned}
$$

In this way

$$
\begin{aligned}
& -\sum_{n=0}^{N-1} \sum_{K \in \mathcal{T}} \int_{K}\left(\eta\left(u_{K}^{n+1}\right)-\eta\left(u_{K}^{n}\right)\right) \varphi(x, n k) d x \\
& +\sum_{n=0}^{N-1} \sum_{K \in \mathcal{T}} \int_{n k}^{(n+1) k} \int_{K} \Phi\left(u_{K}^{n}\right) \vec{v} \cdot \nabla_{x} \varphi(x, n k) d x d t \\
& +\sum_{n=0}^{N-1} \sum_{K \in \mathcal{T}} \int_{n k}^{(n+1) k} \int_{K} \eta^{\prime}\left(u_{K}^{n}\right) g\left(u_{K}^{n}\right) \varphi(x, n k) d x d W(t) \\
& +\frac{1}{2} \sum_{n=0}^{N-1} \sum_{K \in \mathcal{T}} \int_{n k}^{(n+1) k} \int_{K} \eta^{\prime \prime}\left(u_{K}^{n}\right) g^{2}\left(u_{K}^{n}\right) \varphi(x, n k) d x d t \\
& \geqslant \quad R^{h, k},
\end{aligned}
$$

which is exactly inequality $(29)$, where

$$
R^{h, k}=B^{h, k}-\tilde{B}^{h, k}+\bar{B}^{h, k}-B_{1}^{h, k}+\left(\tilde{C}^{h, k}-C^{h, k}\right)+\left(\tilde{D}^{h, k}-D^{h, k}\right) .
$$

STEP II: Convergence of $R_{h, k}$

In this second step, we show that for any measurable set $A$,

$$
E\left[\mathbb{1}_{A} R_{h, k}\right] \underset{h \rightarrow 0}{\rightarrow} 0 .
$$

To do this, we show that all the following quantities converge to 0 :

$$
E\left[\mathbb{1}_{A}\left(B^{h, k}-\tilde{B}^{h, k}\right)\right], E\left[\mathbb{1}_{A}\left(\bar{B}^{h, k}-B_{1}^{h, k}\right)\right], E\left[\mathbb{1}_{A}\left(\tilde{C}^{h, k}-C^{h, k}\right)\right] \text { and } E\left[\mathbb{1}_{A}\left(\tilde{D}^{h, k}-D^{h, k}\right)\right] .
$$

II.1 Convergence of $E\left[\mathbb{1}_{A}\left(B^{h, k}-\tilde{B}^{h, k}\right)\right]$

For almost all $\omega \in \Omega, t \in(n k,(n+1) k)$, any $K \in \mathcal{T}$ and any $n \in\{0, \ldots, N-1\}$, there exists $\zeta_{K}^{n}(\omega, t) \in \mathbb{R}$ such that

$$
\eta^{\prime}\left(\bar{u}_{\mathcal{T}, k}(t)\right)-\eta^{\prime}\left(u_{K}^{n}\right)=\eta^{\prime \prime}\left(\zeta_{K}^{n}(\omega, t)\right)\left(\bar{u}_{\mathcal{T}, k}(t)-u_{K}^{n}\right) .
$$

Note that $\eta^{\prime \prime}\left(\zeta_{K}^{n}(\omega, t)\right)$ is measurable with respect to $\omega$ and $t$ since it depends continuously from $\bar{u}_{\mathcal{T}, k}(t)$ and $u_{K}^{n}$. In this way, by denoting $\zeta_{K}^{n}(\omega, t)=\zeta_{K}^{n}(t)$

$$
\begin{aligned}
B^{h, k}-\tilde{B}^{h, k}= & \sum_{n=0}^{N-1} \sum_{K \in \mathcal{T}_{R}} \int_{n k}^{(n+1) k} \frac{1}{|K|} \int_{K}\left[\eta^{\prime}\left(\bar{u}_{\mathcal{T}, k}(t)\right)-\eta^{\prime}\left(u_{K}^{n}\right)\right] \\
& \quad \times \sum_{L \in \mathcal{N}(K)}\left|\sigma_{K, L}\right|\left\{v_{K, L}^{n} F\left(u_{K}^{n}, u_{L}^{n}\right)-v_{L, K}^{n} F\left(u_{L}^{n}, u_{K}^{n}\right)\right\} \varphi(x, n k) d x d t \\
= & \sum_{n=0}^{N-1} \sum_{K \in \mathcal{T}_{R}} \int_{n k}^{(n+1) k} \frac{1}{|K|} \int_{K}\left[\eta^{\prime \prime}\left(\zeta_{K}^{n}(t)\right)\left(\bar{u}_{\mathcal{T}, k}(t)-u_{K}^{n}\right)\right] \\
& \quad \times \sum_{L \in \mathcal{N}(K)}\left|\sigma_{K, L}\right|\left\{v_{K, L}^{n} F\left(u_{K}^{n}, u_{L}^{n}\right)-v_{L, K}^{n} F\left(u_{L}^{n}, u_{K}^{n}\right)\right\} \varphi(x, n k) d x d t \\
= & T_{1}^{h, k}+T_{2}^{h, k},
\end{aligned}
$$


where

$$
\begin{aligned}
T_{1}^{h, k}=- & \sum_{n=0}^{N-1} \sum_{K \in \mathcal{T}_{R}} \int_{n k}^{(n+1) k} \frac{1}{|K|} \int_{K} \eta^{\prime \prime}\left(\zeta_{K}^{n}(t)\right) \frac{t-n k}{|K|} \varphi(x, n k) d x d t \\
& \times\left(\sum_{L \in \mathcal{N}(K)}\left|\sigma_{K, L}\right|\left\{v_{K, L}^{n}\left(F\left(u_{K}^{n}, u_{L}^{n}\right)-f\left(u_{K}^{n}\right)\right)-v_{L, K}^{n}\left(F\left(u_{L}^{n}, u_{K}^{n}\right)-f\left(u_{K}^{n}\right)\right)\right\}\right)^{2}
\end{aligned}
$$

and

$$
\begin{aligned}
T_{2}^{h, k}= & +\sum_{n=0}^{N-1} \sum_{K \in \mathcal{T}_{R}} \int_{n k}^{(n+1) k} \frac{1}{|K|} \int_{K}\left\{\eta^{\prime \prime}\left(\zeta_{K}^{n}(t)\right) g\left(u_{K}^{n}\right)(W(t)-W(n k))\right\} \\
& \times \sum_{L \in \mathcal{N}(K)}\left|\sigma_{K, L}\right|\left\{v_{K, L}^{n} F\left(u_{K}^{n}, u_{L}^{n}\right)-v_{L, K}^{n} F\left(u_{L}^{n}, u_{K}^{n}\right)\right\} \varphi(x, n k) d x d t .
\end{aligned}
$$

- Let $A$ be a measurable set, we first study $E\left[\mathbb{1}_{A} T_{1}^{h, k}\right]$.

Note that here the assumption $k / h \rightarrow 0$ as $h \rightarrow 0$ is crucial. Using Cauchy-Schwarz inequality, Assumption (4) on the mesh and the first estimate of Proposition 2, we get

$$
\begin{aligned}
\left|E\left[\mathbb{1}_{A} T_{1}^{h, k}\right]\right|= & \mid E\left[\mathbb{1}_{A} \sum_{n=0}^{N-1} \sum_{K \in \mathcal{T}_{R}} \int_{n k}^{(n+1) k} \frac{1}{|K|} \int_{K} \eta^{\prime \prime}\left(\zeta_{K}^{n}(t)\right) \frac{t-n k}{|K|} \varphi(x, n k) d x d t\right. \\
& \left.\times\left[\sum_{L \in \mathcal{N}(K)}\left|\sigma_{K, L}\right|\left\{v_{K, L}^{n}\left(F\left(u_{K}^{n}, u_{L}^{n}\right)-f\left(u_{K}^{n}\right)\right)-v_{L, K}^{n}\left(F\left(u_{L}^{n}, u_{K}^{n}\right)-f\left(u_{K}^{n}\right)\right)\right\}\right)^{2}\right] \mid \\
\leqslant & \left\|\eta^{\prime \prime}\right\|_{\infty}\|\varphi\|_{\infty} \sum_{n=0}^{N-1} \sum_{K \in \mathcal{T}_{R}} \frac{k^{2}}{|K|}\left(\sum_{L \in \mathcal{N}(K)}\left|\sigma_{K, L}\right|\left(v_{K, L}^{n}+v_{L, K}^{n}\right)\right) \\
& \times E\left[\sum_{L \in \mathcal{N}(K)}\left|\sigma_{K, L}\right|\left\{v_{K, L}^{n}\left(F\left(u_{K}^{n}, u_{L}^{n}\right)-f\left(u_{K}^{n}\right)\right)^{2}+v_{L, K}^{n}\left(F\left(u_{L}^{n}, u_{K}^{n}\right)-f\left(u_{K}^{n}\right)\right)^{2}\right\}\right] \\
\leqslant & \left\|\eta^{\prime \prime}\right\|_{\infty}\|\varphi\|_{\infty} \sum_{n=0}^{N-1} \sum_{K \in \mathcal{T}_{R}} \frac{k^{2}}{|K|} V|\partial K| \\
& \times E\left[\sum_{L \in \mathcal{N}(K)}\left|\sigma_{K, L}\right|\left\{v_{K, L}^{n}\left(F\left(u_{K}^{n}, u_{L}^{n}\right)-f\left(u_{K}^{n}\right)\right)^{2}+v_{L, K}^{n}\left(F\left(u_{L}^{n}, u_{K}^{n}\right)-f\left(u_{K}^{n}\right)\right)^{2}\right\}\right] \\
\leqslant & C_{1}\left\|\eta^{\prime \prime}\right\|_{\infty}\|\varphi\|_{\infty} \frac{k}{\bar{\alpha}^{2} h} V \\
\rightarrow & 0 \text { as }\left(h, \frac{k}{h}\right) \rightarrow(0,0),
\end{aligned}
$$

where $C_{1}$ is the constant appearing the first inequality of Proposition 2

- Let us now estimate $E\left[\mathbb{1}_{A} T_{2}^{h, k}\right]$.

Let $C$ be a constant depending only on $\varphi, \eta, C_{g}, T, u_{0}, \xi, F_{1}, F_{2}$ whose value may change from one line to another. Using Cauchy-Schwarz inequality, Assumption (4) on the mesh and then 
Proposition 2

$$
\begin{aligned}
& \left(E\left[\mathbb{1}_{A} T_{2}^{h, k}\right]\right)^{2} \\
& =\left(E \left[\mathbb{1}_{A} \sum_{n=0}^{N-1} \sum_{K \in \mathcal{T}_{R}} \int_{n k}^{(n+1) k} \frac{1}{|K|} \int_{K}\left\{\eta^{\prime \prime}\left(\zeta_{K}^{n}(t)\right) g\left(u_{K}^{n}\right)(W(t)-W(n k))\right\}\right.\right. \\
& \left.\left.\times \sum_{L \in \mathcal{N}(K)}\left|\sigma_{K, L}\right|\left\{v_{K, L}^{n} F\left(u_{K}^{n}, u_{L}^{n}\right)-v_{L, K}^{n} F\left(u_{L}^{n}, u_{K}^{n}\right)\right\} \varphi(x, n k) d x d t\right]\right)^{2} \\
& \leqslant E\left[\sum_{n=0}^{N-1} \sum_{K \in \mathcal{T}_{R}} \int_{n k}^{(n+1) k} \int_{K}\left|\mathbb{1}_{A} \eta^{\prime \prime}\left(\zeta_{K}^{n}(t)\right) g\left(u_{K}^{n}\right) \varphi(x, n k)\right|^{2} d x d t\right] \\
& \times E\left[\sum_{n=0}^{N-1} \sum_{K \in \mathcal{T}_{R}} \int_{n k}^{(n+1) k} \frac{1}{|K|}\left((W(t)-W(n k)) \sum_{L \in \mathcal{N}(K)}\left|\sigma_{K, L}\right|\left\{v_{K, L}^{n} F\left(u_{K}^{n}, u_{L}^{n}\right)-v_{L, K}^{n} F\left(u_{L}^{n}, u_{K}^{n}\right)\right\}\right)^{2} d t\right] \\
& \leqslant\|\varphi\|_{\infty}^{2}\left\|\eta^{\prime \prime}\right\|_{\infty}^{2} C_{g}^{2}\left\|u_{\mathcal{T}, k}\right\|_{L^{2}(\Omega \times Q)}^{2} \\
& \times E\left[\sum_{n=0}^{N-1} \sum_{K \in \mathcal{T}_{R}} \int_{n k}^{(n+1) k} \frac{1}{|K|}\left((W(t)-W(n k)) \sum_{L \in \mathcal{N}(K)}\left|\sigma_{K, L}\right|\left\{v_{K, L}^{n} F\left(u_{K}^{n}, u_{L}^{n}\right)-v_{L, K}^{n} F\left(u_{L}^{n}, u_{K}^{n}\right)\right\}\right)^{2} d t\right] \\
& \leqslant C \sum_{n=0}^{N-1} \sum_{K \in \mathcal{T}_{R}} \int_{n k}^{(n+1) k} \frac{k}{|K|} E\left[\left(\sum_{L \in \mathcal{N}(K)}\left|\sigma_{K, L}\right|\left\{v_{K, L}^{n} F\left(u_{K}^{n}, u_{L}^{n}\right)-v_{L, K}^{n} F\left(u_{L}^{n}, u_{K}^{n}\right)\right\}\right)^{2}\right] d t \\
& \leqslant C \sum_{n=0}^{N-1} \sum_{K \in \mathcal{T}_{R}} \frac{k^{2}}{|K|} E\left[\left(\sum_{L \in \mathcal{N}(K)}\left|\sigma_{K, L}\right|\left\{v_{K, L}^{n}\left(F\left(u_{K}^{n}, u_{L}^{n}\right)-f\left(u_{K}^{n}\right)\right)-v_{L, K}^{n}\left(F\left(u_{L}^{n}, u_{K}^{n}\right)-f\left(u_{K}^{n}\right)\right)\right\}\right)^{2}\right] \\
& \leqslant C \sum_{n=0}^{N-1} \sum_{K \in \mathcal{T}_{R}} \frac{k^{2}}{|K|}\left(\sum_{L \in \mathcal{N}(K)}\left|\sigma_{K, L}\right|\left(v_{K, L}^{n}+v_{L, K}^{n}\right)\right) \\
& \times E\left[\sum_{L \in \mathcal{N}(K)}\left|\sigma_{K, L}\right|\left\{v_{K, L}^{n}\left(F\left(u_{K}^{n}, u_{L}^{n}\right)-f\left(u_{K}^{n}\right)\right)^{2}+v_{L, K}^{n}\left(F\left(u_{L}^{n}, u_{K}^{n}\right)-f\left(u_{K}^{n}\right)\right)^{2}\right\}\right] \\
& \leqslant C V \frac{k}{\bar{\alpha}^{2} h} \\
& \rightarrow 0 \text { as }\left(h, \frac{k}{h}\right) \rightarrow(0,0) \text {. }
\end{aligned}
$$

II.2 Convergence of $E\left[\mathbb{1}_{A}\left(\bar{B}^{h, k}-B_{1}^{h, k}\right)\right]$

To begin with, we split $\bar{B}^{h, k}$ and $B_{1}^{h, k}$ into the sum of two terms. Using again the fact that

$$
\sum_{L \in \mathcal{N}(K)}\left|\sigma_{K, L}\right|\left(v_{K, L}^{n}-v_{L, K}^{n}\right) \Phi\left(u_{K}^{n}\right)=0,
$$

we can rewrite

$\bar{B}^{h, k}=\sum_{n=0}^{N-1} \sum_{K \in \mathcal{T}_{R}} \frac{k}{|K|} \sum_{L \in \mathcal{N}(K)}\left|\sigma_{K, L}\right|\left\{v_{K, L}^{n}\left(G\left(u_{K}^{n}, u_{L}^{n}\right)-\Phi\left(u_{K}^{n}\right)\right)-v_{L, K}^{n}\left(G\left(u_{L}^{n}, u_{K}^{n}\right)-\Phi\left(u_{K}^{n}\right)\right)\right\} \int_{K} \varphi(x, n k) d x$ in the following way $\bar{B}^{h, k}=\bar{T}_{1}^{h, k}-\bar{T}_{2}^{h, k}$, where

$$
\begin{aligned}
& \bar{T}_{1}^{h, k}=\sum_{n=0}^{N-1} \sum_{(K, L) \in \mathfrak{T}_{n}^{R}} \frac{k}{|K|}\left|\sigma_{K, L}\right|\left\{v_{K, L}^{n}\left(G\left(u_{K}^{n}, u_{L}^{n}\right)-\Phi\left(u_{K}^{n}\right)\right)-v_{L, K}^{n}\left(G\left(u_{L}^{n}, u_{K}^{n}\right)-\Phi\left(u_{K}^{n}\right)\right)\right\} \int_{K} \varphi(x, n k) d x \\
& \text { and } \\
& \bar{T}_{2}^{h, k}=\sum_{n=0}^{N-1} \sum_{(K, L) \in \mathfrak{T}_{n}^{R}} \frac{k}{|L|}\left|\sigma_{K, L}\right|\left\{v_{K, L}^{n}\left(G\left(u_{K}^{n}, u_{L}^{n}\right)-\Phi\left(u_{L}^{n}\right)\right)-v_{L, K}^{n}\left(G\left(u_{L}^{n}, u_{K}^{n}\right)-\Phi\left(u_{L}^{n}\right)\right)\right\} \int_{L} \varphi(x, n k) d x .
\end{aligned}
$$


Similarly,

$$
\begin{aligned}
B_{1}^{h, k}= & -\sum_{n=0}^{N-1} \sum_{K \in \mathcal{T}_{R}} \int_{n k}^{(n+1) k} \int_{K} \Phi\left(u_{K}^{n}\right) \vec{v}(x, t) \cdot \nabla x \varphi(x, n k) d x d t \\
= & -\sum_{n=0}^{N-1} \sum_{K \in \mathcal{T}_{R}} \int_{n k}^{(n+1) k} \int_{K} \Phi\left(u_{K}^{n}\right) \operatorname{div}[\vec{v}(x, t) \varphi(x, n k)] d x d t \\
= & -\sum_{n=0}^{N-1} \sum_{K \in \mathcal{T}_{R}} \sum_{L \in \mathcal{N}(K)} \int_{n k}^{(n+1) k} \int_{\sigma_{K, L}} \Phi\left(u_{K}^{n}\right) \vec{v}(x, t) \cdot n_{K, L} \varphi(x, n k) d \gamma(x) d t \\
= & \sum_{n=0}^{N-1} \sum_{(K, L) \in \mathfrak{T}_{n}^{R}} \int_{n k}^{(n+1) k} \int_{\sigma_{K, L}}-\varphi(x, n k) \Phi\left(u_{K}^{n}\right) \vec{v}(x, t) \cdot n_{K, L} d \gamma(x) d t \\
& -\sum_{n=0}^{N-1} \sum_{(K, L) \in \mathfrak{T}_{n}^{R}} \int_{n k}^{(n+1) k} \int_{\sigma_{K, L}}-\varphi(x, n k) \Phi\left(u_{L}^{n}\right) \vec{v}(x, t) \cdot n_{K, L} d \gamma(x) d t \\
= & T_{1}^{h, k}-T_{2}^{h, k},
\end{aligned}
$$

where

$$
\begin{aligned}
& T_{1}^{h, k}=\sum_{n=0}^{N-1} \sum_{(K, L) \in \mathfrak{T}_{n}^{R}} \int_{n k}^{(n+1) k} \int_{\sigma_{K, L}}\left\{v_{K, L}^{n} G\left(u_{K}^{n}, u_{L}^{n}\right)-v_{L, K}^{n} G\left(u_{L}^{n}, u_{K}^{n}\right)-\Phi\left(u_{K}^{n}\right) \vec{v}(x, t) \cdot n_{K, L}\right\} \varphi(x, n k) d \gamma(x) d t \\
& \text { and } \\
& T_{2}^{h, k}=\sum_{n=0}^{N-1} \sum_{(K, L) \in \mathfrak{T}_{n}^{R}} \int_{n k}^{(n+1) k} \int_{\sigma_{K, L}}\left\{v_{K, L}^{n} G\left(u_{K}^{n}, u_{L}^{n}\right)-v_{L, K}^{n} G\left(u_{L}^{n}, u_{K}^{n}\right)-\Phi\left(u_{L}^{n}\right) \vec{v}(x, t) \cdot n_{K, L}\right\} \varphi(x, n k) d \gamma(x) d t .
\end{aligned}
$$

Now our aim is to estimate $\left|\bar{T}_{1}^{h, k}-T_{1}^{h, k}\right|$ and $\left|\bar{T}_{2}^{h, k}-T_{2}^{h, k}\right|$. To do this, we first note that these quantities can be rewritten in the following way :

$$
\begin{aligned}
\bar{T}_{1}^{h, k}-T_{1}^{h, k}= & \sum_{n=0}^{N-1} \sum_{(K, L) \in \mathfrak{T}_{n}^{R}} k\left|\sigma_{K, L}\right|\left\{v_{K, L}^{n}\left(G\left(u_{K}^{n}, u_{L}^{n}\right)-\Phi\left(u_{K}^{n}\right)\right)-v_{L, K}^{n}\left(G\left(u_{L}^{n}, u_{K}^{n}\right)-\Phi\left(u_{K}^{n}\right)\right)\right\} \\
& \times\left\{\frac{1}{|K|} \int_{K} \varphi(y, n k) d y-\frac{1}{\left|\sigma_{K, L}\right|} \int_{\sigma_{K, L}} \varphi(x, n k) d \gamma(x)\right\} \\
& +\sum_{n=0}^{N-1} \sum_{(K, L) \in \mathfrak{T}_{n}^{R}} k \int_{\sigma_{K, L}}\left\{v_{K, L}^{n}\left(G\left(u_{K}^{n}, u_{L}^{n}\right)-\Phi\left(u_{K}^{n}\right)\right)-v_{L, K}^{n}\left(G\left(u_{L}^{n}, u_{K}^{n}\right)-\Phi\left(u_{K}^{n}\right)\right)\right\} \varphi(x, n k) d \gamma(x) \\
& -\sum_{n=0}^{N-1} \sum_{(K, L) \in \mathfrak{T}_{n}^{R}} \int_{n k}^{(n+1) k} \int_{\sigma_{K, L}}\left\{v_{K, L}^{n} G\left(u_{K}^{n}, u_{L}^{n}\right)-v_{L, K}^{n} G\left(u_{L}^{n}, u_{K}^{n}\right)-\Phi\left(u_{K}^{n}\right) \vec{v}(x, s) \cdot n_{K, L}\right\} \varphi(x, n k) d \gamma(x) d s \\
= & \sum_{n=0}^{N-1} \sum_{(K, L) \in \mathfrak{T}_{n}^{R}} k\left|\sigma_{K, L}\right|\left\{v_{K, L}^{n}\left(G\left(u_{K}^{n}, u_{L}^{n}\right)-\Phi\left(u_{K}^{n}\right)\right)-v_{L, K}^{n}\left(G\left(u_{L}^{n}, u_{K}^{n}\right)-\Phi\left(u_{K}^{n}\right)\right)\right\} \\
& +\sum_{n=0}^{N-1} \sum_{(K, L) \in \mathfrak{T}_{n}^{R}} \Phi\left(u_{K}^{n}\right) \int_{n k}^{(n+1) k} \int_{\sigma_{K, L}}\left[\vec{v}(x, s) \cdot n_{K, L}-\left(v_{K, L}^{n}-v_{L, K}^{n}\right)\right] \varphi(x, n k) d \gamma(x) d s
\end{aligned}
$$

and we also have

$$
\begin{aligned}
\bar{T}_{2}^{h, k}-T_{2}^{h, k}= & \sum_{n=0}^{N-1} \sum_{(K, L) \in \mathfrak{T}_{n}^{R}} k\left|\sigma_{K, L}\right|\left\{v_{K, L}^{n}\left(G\left(u_{K}^{n}, u_{L}^{n}\right)-\Phi\left(u_{L}^{n}\right)\right)-v_{L, K}^{n}\left(G\left(u_{L}^{n}, u_{K}^{n}\right)-\Phi\left(u_{L}^{n}\right)\right)\right\} \\
& \times\left\{\frac{1}{|L|} \int_{L} \varphi(y, n k) d y-\frac{1}{\left|\sigma_{K, L}\right|} \int_{\sigma_{K, L}} \varphi(x, n k) d \gamma(x)\right\} \\
& +\sum_{n=0}^{N-1} \sum_{(K, L) \in \mathfrak{T}_{n}^{R}} \Phi\left(u_{L}^{n}\right) \int_{n k}^{(n+1) k} \int_{\sigma_{K, L}}\left[\vec{v}(x, s) \cdot n_{K, L}-\left(v_{K, L}^{n}-v_{L, K}^{n}\right)\right] \varphi(x, n k) d \gamma(x) d s .
\end{aligned}
$$

In order to control $\left|\bar{T}_{1}^{h, k}-T_{1}^{h, k}\right|$ and $\left|\bar{T}_{2}^{h, k}-T_{2}^{h, k}\right|$, we need to bound the following quantities:

$$
\begin{aligned}
& G\left(u_{K}^{n}, u_{L}^{n}\right)-\Phi\left(u_{K}^{n}\right), G\left(u_{K}^{n}, u_{L}^{n}\right)-\Phi\left(u_{L}^{n}\right), \\
& G\left(u_{L}^{n}, u_{K}^{n}\right)-\Phi\left(u_{K}^{n}\right), G\left(u_{L}^{n}, u_{K}^{n}\right)-\Phi\left(u_{L}^{n}\right), \\
& \frac{1}{\left|\sigma_{K, L}\right|} \int_{\sigma_{K, L}} \varphi(x, n k) d \gamma(x)-\frac{1}{|K|} \int_{K} \varphi(y, n k) d y, \\
\text { and } \quad & \sum_{n=0}^{N-1} \sum_{(K, L) \in \mathfrak{T}_{n}^{R}} \Phi\left(u_{K}^{n}\right) \int_{n k}^{(n+1) k} \int_{\sigma_{K, L}}\left[\vec{v}(x, s) \cdot n_{K, L}-\left(v_{K, L}^{n}-v_{L, K}^{n}\right)\right] \varphi(x, n k) d \gamma(x) d s .
\end{aligned}
$$


- Let us begin with $G\left(u_{K}^{n}, u_{L}^{n}\right)-\Phi\left(u_{K}^{n}\right)$, and then similar quantities. Set $(K, L) \in \mathfrak{T}_{n}$, we then have $u_{K}^{n}>u_{L}^{n}$ which implies that $f\left(s\left(u_{K}^{n}, u_{L}^{n}\right)\right)=\max _{t \in\left[u_{L}^{n}, u_{K}^{n}\right]} f(t)$ and hence

$$
\begin{aligned}
G\left(u_{K}^{n}, u_{L}^{n}\right)-\Phi\left(u_{K}^{n}\right) & =\Phi\left(s\left(u_{K}^{n}, u_{L}^{n}\right)\right)-\Phi\left(u_{K}^{n}\right) \\
& =\int_{u_{K}^{n}}^{s\left(u_{K}^{n}, u_{L}^{n}\right)} \eta^{\prime}(t) f^{\prime}(t) d t
\end{aligned}
$$

First case : $\int_{u_{K}^{n}}^{s\left(u_{K}^{n}, u_{L}^{n}\right)} \eta^{\prime}(t) f^{\prime}(t) d t<0$.

Using an integration by parts formula, we get with $d$ such that $f(d)=\min _{t \in\left[s\left(u_{K}^{n}, u_{L}^{n}\right), u_{K}^{n}\right]} f(t)$ :

$$
\begin{aligned}
\int_{u_{K}^{n}}^{s\left(u_{K}^{n}, u_{L}^{n}\right)} \eta^{\prime}(t) f^{\prime}(t) d t & =\int_{s\left(u_{K}^{n}, u_{L}^{n}\right)}^{u_{K}^{n}} \eta^{\prime \prime}(t)[f(t)-f(d)] d t-\left[\eta^{\prime}(t)(f(t)-f(d))\right]_{s\left(u_{K}^{n}, u_{L}^{n}\right)}^{u_{K}^{n}} \\
& \geqslant-\eta^{\prime}\left(u_{K}^{n}\right)\left[f\left(u_{K}^{n}\right)-f(d)\right]+\eta^{\prime}\left(s\left(u_{K}^{n}, u_{L}^{n}\right)\right)\left[f\left(s\left(u_{K}^{n}, u_{L}^{n}\right)\right)-f(d)\right] \\
& \geqslant-\left\|\eta^{\prime}\right\|_{\infty}\left|f\left(u_{K}^{n}\right)-f(d)\right|-\left\|\eta^{\prime}\right\|_{\infty}\left|f\left(s\left(u_{K}^{n}, u_{L}^{n}\right)\right)-f(d)\right| \\
& \geqslant-2\left\|\eta^{\prime}\right\|_{\infty}\left|f\left(s\left(u_{K}^{n}, u_{L}^{n}\right)\right)-f(d)\right| \\
& \geqslant-2\left\|\eta^{\prime}\right\|_{\infty} \max _{u_{L}^{n} \leqslant c \leqslant d \leqslant u_{K}^{n}}|F(d, c)-F(d, d)|,
\end{aligned}
$$

since $f\left(s\left(u_{K}^{n}, u_{L}^{n}\right)\right)=\max _{t \in\left[u_{L}^{n}, u_{K}^{n}\right]} f(t)=\max _{t \in\left[s\left(u_{K}^{n}, u_{L}^{n}\right), d\right]} f(t)=F\left(d, s\left(u_{K}^{n}, u_{L}^{n}\right)\right)$.

Second case : $\int_{u_{K}^{n}}^{s\left(u_{K}^{n}, u_{L}^{n}\right)} \eta^{\prime}(t) f^{\prime}(t) d t>0$.

Similarly we have:

$$
\begin{aligned}
\int_{u_{K}^{n}}^{s\left(u_{K}^{n}, u_{L}^{n}\right)} \eta^{\prime}(t) f^{\prime}(t) d t & \left.=\int_{s\left(u_{K}^{n}, u_{L}^{n}\right)}^{u_{K}^{n}} \eta^{\prime \prime}(t)\left[f(t)-f\left(s\left(u_{K}^{n}, u_{L}^{n}\right)\right)\right] d t-\left[\eta^{\prime}(t)\left(f(t)-f\left(s\left(u_{K}^{n}, u_{L}^{n}\right)\right)\right)\right]_{s}^{u_{K}^{n}} u_{K}^{n}, u_{L}^{n}\right) \\
& \leqslant \eta^{\prime}\left(u_{K}^{n}\right)\left[f\left(s\left(u_{K}^{n}, u_{L}^{n}\right)\right)-f\left(u_{K}^{n}\right)\right] \\
& \leqslant\left\|\eta^{\prime}\right\|_{\infty}\left|F\left(u_{K}^{n}, u_{K}^{n}\right)-F\left(u_{K}^{n}, s\left(u_{K}^{n}, u_{L}^{n}\right)\right)\right| \\
& \leqslant\left\|\eta^{\prime}\right\|_{\infty} \max _{u_{L}^{n} \leqslant c \leqslant d \leqslant u_{K}^{n}}|F(d, c)-f(d)|,
\end{aligned}
$$

since $f\left(s\left(u_{K}^{n}, u_{L}^{n}\right)\right)=\max _{t \in\left[u_{L}^{n}, u_{K}^{n}\right]} f(t)=\max _{t \in\left[s\left(u_{K}^{n}, u_{L}^{n}\right), u_{K}^{n}\right]} f(t)=F\left(u_{K}^{n}, s\left(u_{K}^{n}, u_{L}^{n}\right)\right)$.

We deduce that in both cases we have

$$
\left|G\left(u_{K}^{n}, u_{L}^{n}\right)-\Phi\left(u_{K}^{n}\right)\right| \leqslant 2\left\|\eta^{\prime}\right\|_{\infty_{u_{L}^{n} \leqslant c \leqslant d \leqslant u_{K}^{n}}}|F(d, c)-f(d)| .
$$

Similarly, we can show using the same technics that for any $(K, L) \in \mathfrak{T}_{n}^{R}$

$$
\begin{gathered}
\left|G\left(u_{L}^{n}, u_{K}^{n}\right)-\Phi\left(u_{K}^{n}\right)\right| \leqslant 2\left\|\eta^{\prime}\right\|_{\infty} \max _{u_{L}^{n} \leqslant c \leqslant d \leqslant u_{K}^{n}}|F(c, d)-f(d)| \\
\left|G\left(u_{K}^{n}, u_{L}^{n}\right)-\Phi\left(u_{L}^{n}\right)\right| \leqslant 2\left\|\eta^{\prime}\right\|_{\infty} \max _{u_{L}^{n} \leqslant c \leqslant d \leqslant u_{K}^{n}}|F(d, c)-f(c)| \\
\left|G\left(u_{L}^{n}, u_{K}^{n}\right)-\Phi\left(u_{L}^{n}\right)\right| \leqslant 2\left\|\eta^{\prime}\right\|_{\infty} \max _{u_{L}^{n} \leqslant c \leqslant d \leqslant u_{K}^{n}}|F(c, d)-f(c)| .
\end{gathered}
$$

- We are now going to estimate $\frac{1}{\left|\sigma_{K, L}\right|} \int_{\sigma_{K, L}} \varphi(x, n k) d \gamma(x)-\frac{1}{|K|} \int_{K} \varphi(y, n k) d y$. Using the regularity of $\varphi$ we get the following bound:

$$
\left|\frac{1}{\left|\sigma_{K, L}\right|} \int_{\sigma_{K, L}} \varphi(x, n k) d \gamma(x)-\frac{1}{|K|} \int_{K} \varphi(y, n k) d y\right| \leqslant 2 h\left\|\nabla_{x} \varphi\right\|_{\infty} .
$$

- Let us now bound $\sum_{n=0}^{N-1} \sum_{(K, L) \in \mathfrak{T}_{n}^{R}} \Phi\left(u_{K}^{n}\right) \int_{n k}^{(n+1) k} \int_{\sigma_{K, L}}\left[\vec{v}(x, s) \cdot n_{K, L}-\left(v_{K, L}^{n}-v_{L, K}^{n}\right)\right] \varphi(x, n k) d \gamma(x) d s$.

First note that this term is equal to

$$
\sum_{n=0}^{N-1} \sum_{(K, L) \in \mathfrak{T}_{n}^{R}} \Phi\left(u_{K}^{n}\right) \int_{n k}^{(n+1) k} \int_{\sigma_{K, L}}\left[\frac{1}{k\left|\sigma_{K, L}\right|} \int_{n k}^{(n+1) k} \int_{\sigma_{K, L}}\{\vec{v}(x, s)-\vec{v}(\bar{x}, t)\} \cdot n_{K, L} d \gamma(\bar{x}) d t\right] \varphi(x, n k) d \gamma(x) d s
$$

and thanks to the regularity of $\vec{v}$, we deduce that there exists a constant $c(\vec{v})$ only depending on $\vec{v}$ such that

$$
\frac{1}{k\left|\sigma_{K, L}\right|}\left|\int_{n k}^{(n+1) k} \int_{\sigma_{K, L}}\{\vec{v}(x, s)-\vec{v}(\bar{x}, t)\} \cdot n_{K, L} d \gamma(\bar{x}) d t\right| \leqslant c(\vec{v})(k+h) .
$$


Hence, by denoting $x_{\sigma}$ the center of the edge $\sigma_{K, L}$

$$
\begin{aligned}
& \int_{n k}^{(n+1) k} \int_{\sigma_{K, L}}\left\{\vec{v}(x, s) \cdot n_{K, L}-\left(v_{K, L}^{n}-v_{L, K}^{n}\right)\right\} \varphi(x, n k) d \gamma(x) d s \\
& =\int_{n k}^{(n+1) k} \int_{\sigma_{K, L}}\left(\frac{1}{k\left|\sigma_{K, L}\right|} \int_{\sigma_{K, L}} \int_{n k}^{(n+1) k}\{\vec{v}(x, s)-\vec{v}(\bar{x}, t)\} \cdot n_{K, L} d \gamma(\bar{x}) d t\right)\left[\varphi(x, n k)-\varphi\left(x_{\sigma}, n k\right)\right] d \gamma(x) d s,
\end{aligned}
$$

and we deduce since

$$
\varphi\left(x_{\sigma}, n k\right)\left(\frac{1}{k\left|\sigma_{K, L}\right|} \int_{n k}^{(n+1) k} \int_{\sigma_{K, L}} \int_{n k}^{(n+1) k} \int_{\sigma_{K, L}}\{\vec{v}(x, s)-\vec{v}(\bar{x}, t)\} . n_{K, L} d \gamma(x) d s d \gamma(\bar{x}) d t\right)=0
$$

that

$$
\left|\int_{n k}^{(n+1) k} \int_{\sigma_{K, L}}\left\{\vec{v}(x, s) \cdot n_{K, L}-\left(v_{K, L}^{n}-v_{L, K}^{n}\right)\right\} \varphi(x, n k) d \gamma(x) d s\right| \leqslant c(\vec{v})\left\|\nabla_{x} \varphi\right\|_{\infty} h^{2} k\left|\sigma_{K, L}\right| .
$$

In this way, there exists a constant $C$ depending only on $\vec{v}, \varphi, u_{0}, T, C_{g}, \bar{\alpha}, \eta$ and $f$, whose value may change from one line to another such that

$$
\begin{aligned}
& \left|\sum_{n=0}^{N-1} \sum_{(K, L) \in \mathfrak{T}_{n}^{R}} \Phi\left(u_{K}^{n}\right) \int_{n k}^{(n+1) k} \int_{\sigma_{K, L}}\left[\vec{v}(x, s) \cdot n_{K, L}-\left(v_{K, L}^{n}-v_{L, K}^{n}\right)\right] \varphi(x, n k) d \gamma(x) d s\right| \\
\leqslant & C h^{2} \sum_{n=0}^{N-1} k \sum_{K \in \mathcal{T}_{R}} \sum_{L \in \mathcal{N}(K)}\left|\sigma_{K, L} \| \Phi\left(u_{K}^{n}\right)\right| \\
\leqslant & C h^{2} \sum_{n=0}^{N-1} \sum_{K \in \mathcal{T}_{R}} k\left|\partial K \| u_{K}^{n}\right| \\
\leqslant & \frac{C}{\bar{\alpha}^{2}} h \sum_{n=0}^{N-1} \sum_{K \in \mathcal{T}_{R}} k|K|\left|u_{K}^{n}\right| \\
\leqslant & C h\left\|u_{\mathcal{T}, k}\right\|_{L^{1}(B(0, R) \times(0, T)) .}
\end{aligned}
$$

Similary we get for some constant $C$ depending only on $\vec{v}, \varphi, u_{0}, T, C_{g}, \bar{\alpha}, \eta$ and $f$, whose value may change from one line to another that

$$
\begin{aligned}
& \left|\sum_{n=0}^{N-1} \sum_{(K, L) \in \mathfrak{T}_{n}^{R}} \Phi\left(u_{L}^{n}\right) \int_{n k}^{(n+1) k} \int_{\sigma_{K, L}}\left[\vec{v}(x, s) \cdot n_{K, L}-\left(v_{K, L}^{n}-v_{L, K}^{n}\right)\right] \varphi(x, n k) d \gamma(x) d s\right| \\
\leqslant & C h\left\|u_{\mathcal{T}, k}\right\|_{L^{1}(B(0, R) \times(0, T)) .}
\end{aligned}
$$

- We are now ready to compare $\bar{B}^{h, k}$ to $B_{1}^{h, k}$. We first recall that

$$
\bar{B}^{h, k}-B_{1}^{h, k}=\bar{T}_{1}^{h, k}-T_{1}^{h, k}-\left(\bar{T}_{2}^{h, k}-T_{2}^{h, k}\right) .
$$

Using (36), 37), 40 and 42 we get for some constant $C$ depending only on $\vec{v}, \varphi, u_{0}, T, C_{g}, \bar{\alpha}, \eta, f$ and whose value may change from one line to another

$$
\begin{aligned}
\left|\bar{T}_{1}^{h, k}-T_{1}^{h, k}\right| \leqslant & C \sum_{n=0}^{N-1} \sum_{(K, L) \in \mathfrak{T}_{n}^{R}} k\left|\sigma_{K, L}\right| h\left(v_{K, L}^{n} \max _{u_{L}^{n} \leqslant c \leqslant d \leqslant u_{K}^{n}}|F(d, c)-f(d)|+v_{L, K}^{n} \max _{u_{L}^{n} \leqslant c \leqslant d \leqslant u_{K}^{n}}|F(c, d)-f(d)|\right) \\
& +C h\left\|u_{\mathcal{T}, k}\right\|_{L^{1}(B(0, R) \times(0, T)) .}
\end{aligned}
$$

And using (38), 39, 40 and 43, we get similarly for some constant $C$ depending only on $\vec{v}, \varphi, u_{0}, T, C_{g}, \bar{\alpha}, \eta, f$ and whose value may change from one line to another

$$
\begin{aligned}
\left|\bar{T}_{2}^{h, k}-T_{2}^{h, k}\right| \leqslant & C \sum_{n=0}^{N-1} \sum_{(K, L) \in \mathfrak{T}_{n}^{R}} k\left|\sigma_{K, L}\right| h\left(v_{K, L}^{n} \max _{u_{L}^{n} \leqslant c \leqslant d \leqslant u_{K}^{n}}|F(d, c)-f(c)|+v_{L, K}^{n} \max _{u_{L}^{n} \leqslant c \leqslant d \leqslant u_{K}^{n}}|F(c, d)-f(c)|\right) \\
& +C h\left\|u_{\mathcal{T}, k}\right\|_{L^{1}(B(0, R) \times(0, T)) .}
\end{aligned}
$$

Combining these two inequalities and using Proposition 2, we get for some constant $C$ depending only on $\vec{v}, \varphi, u_{0}, T, C_{g}, \bar{\alpha}, \eta, f$ and whose value may change from one line to another for almost all $\omega$

$$
\begin{aligned}
\left|\bar{B}^{h, k}-B_{1}^{h, k}\right| \leqslant & C \sum_{n=0}^{N-1} \sum_{(K, L) \in \mathfrak{T}_{n}^{R}} k\left|\sigma_{K, L}\right| h\left(v_{K, L}^{n}\left\{\max _{u_{L}^{n} \leqslant c \leqslant d \leqslant u_{K}^{n}}|F(d, c)-f(d)|+\max _{u_{L}^{n} \leqslant c \leqslant d \leqslant u_{K}^{n}}|F(d, c)-f(c)|\right\}\right. \\
& \left.+v_{L, K}^{n}\left\{\max _{u_{L}^{n} \leqslant c \leqslant d \leqslant u_{K}^{n}}|F(c, d)-f(d)|+\max _{u_{L}^{n} \leqslant c \leqslant d \leqslant u_{K}^{n}}|F(c, d)-f(c)|\right\}\right)+C h\left\|u_{\mathcal{T}, k}\right\|_{L^{1}(B(0, R) \times(0, T))} \\
\leqslant & C h^{1 / 2}+C h\left\|u_{\mathcal{T}, k}\right\|_{L^{1}(B(0, R) \times(0, T)) .}
\end{aligned}
$$


Therefore we have that for any measurable set $A, E\left[\mathbb{1}_{A}\left(\bar{B}^{h, k}-B_{1}^{h, k}\right)\right] \underset{h \rightarrow 0}{\rightarrow} 0$.

II.3 Convergence of $E\left[\mathbb{1}_{A}\left(\tilde{C}^{h, k}-C^{h, k}\right)\right]$

$$
\begin{aligned}
\left|E\left[\mathbb{1}_{A}\left(\tilde{C}^{h, k}-C^{h, k}\right)\right]\right|= & \left|E\left[\sum_{K \in \mathcal{T}_{R}} \sum_{n=0}^{N-1} \int_{K} \mathbb{1}_{A} \int_{n k}^{(n+1) k}\left[\eta^{\prime}\left(\bar{u}_{\mathcal{T}, k}(t)\right)-\eta^{\prime}\left(u_{K}^{n}\right)\right] g\left(u_{K}^{n}\right) \varphi(x, n k) d W(t) d x\right]\right| \\
\leqslant & \left|E\left[\sum_{K \in \mathcal{T}_{R}} \sum_{n=0}^{N-1} \int_{K} \mathbb{1}_{A} \int_{n k}^{(n+1) k}\left[\eta^{\prime}\left(\bar{u}_{\mathcal{T}, k}(t)\right)-\eta^{\prime}\left(u_{K}^{n}\right)\right] g\left(u_{K}^{n}\right)\{\varphi(x, n k)-\varphi(x, t)\} d W(t) d x\right]\right| \\
& +\left|E\left[\sum_{K \in \mathcal{T}_{R}} \sum_{n=0}^{N-1} \int_{K} \mathbb{1}_{A} \int_{n k}^{(n+1) k}\left[\eta^{\prime}\left(\bar{u}_{\mathcal{T}, k}(t)\right)-\eta^{\prime}\left(u_{K}^{n}\right)\right] g\left(u_{K}^{n}\right) \varphi(x, t) d W(t) d x\right]\right| \\
= & S_{1}^{h, k}+S_{2}^{h, k} .
\end{aligned}
$$

Using successively Cauchy-Schwarz inequality on $\Omega \times B(0, R)$, Itô isometry and Proposition 1 one gets

$$
\begin{aligned}
S_{1}^{h, k} & =\left|E\left[\sum_{K \in \mathcal{T}_{R}} \sum_{n=0}^{N-1} \int_{K} \mathbb{1}_{A} \int_{n k}^{(n+1) k}\left\{\eta^{\prime}\left(\bar{u}_{\mathcal{T}, k}(t)\right)-\eta^{\prime}\left(u_{K}^{n}\right)\right\} g\left(u_{K}^{n}\right)\{\varphi(x, n k)-\varphi(x, t)\} d W(t) d x\right]\right| \\
& \leqslant \sqrt{|B(0, R)|} \sum_{n=0}^{N-1}\left[\sum_{K \in \mathcal{T}_{R}} \int_{K} E\left[\left(\int_{n k}^{(n+1) k}\left\{\eta^{\prime}\left(\bar{u}_{\mathcal{T}, k}(t)\right)-\eta^{\prime}\left(u_{K}^{n}\right)\right\} g\left(u_{K}^{n}\right)\{\varphi(x, n k)-\varphi(x, t)\} d W(t)\right)^{2}\right] d x\right]^{1 / 2} \\
& =\sqrt{|B(0, R)|} \sum_{n=0}^{N-1}\left[\sum_{K \in \mathcal{T}_{R}} \int_{K} \int_{n k}^{(n+1) k} E\left[\left\{\eta^{\prime}\left(\bar{u}_{\mathcal{T}, k}(t)\right)-\eta^{\prime}\left(u_{K}^{n}\right)\right\}^{2} g^{2}\left(u_{K}^{n}\right)\{\varphi(x, n k)-\varphi(x, t)\}^{2}\right] d t d x\right]^{1 / 2} \\
& \leqslant \sqrt{k} \sqrt{|B(0, R)|} 2 C_{g}\left\|\varphi_{t}\right\|_{\infty}\left\|\eta^{\prime}\right\|_{\infty} \sum_{n=0}^{N-1} k\left(\sum_{K \in \mathcal{T}_{R}}|K| E\left[\left(u_{K}^{n}\right)^{2}\right]\right)^{1 / 2} \\
& \leqslant \sqrt{k} \sqrt{|B(0, R)|} 2 C_{g}\left\|\varphi_{t}\right\|_{\infty}\left\|\eta^{\prime}\right\|_{\infty} T e^{T C_{g}^{2} / 2}\left\|u_{0}\right\|_{L^{2}\left(\mathbb{R}^{d}\right) \underset{h \rightarrow 0}{\rightarrow} 0 .} 0 .
\end{aligned}
$$

Note that here Assumption $H_{6}$ on the function $g$ is important:

$$
\begin{aligned}
\left(S_{2}^{h, k}\right)^{2} & =\left|E\left[\sum_{n=0}^{N-1} \sum_{K \in \mathcal{T}_{R}} \mathbb{1}_{A} \int_{K} \int_{n k}^{(n+1) k}\left\{\eta^{\prime}\left(\bar{u}_{\mathcal{T}, k}(t)\right)-\eta^{\prime}\left(u_{K}^{n}\right)\right\} g\left(u_{K}^{n}\right) \varphi(x, t) d W(t) d x\right]\right|^{2} \\
& =\left|E\left[\mathbb{1}_{A} \int_{B(0, R)} \int_{0}^{T}\left\{\eta^{\prime}\left(\bar{u}_{\mathcal{T}, k}\right)-\eta^{\prime}\left(u_{\mathcal{T}, k}\right)\right\} g\left(u_{\mathcal{T}, k}\right) \varphi(x, t) d W(t) d x\right]\right|^{2} \\
& \leqslant|B(0, R)| \int_{B(0, R)} E\left[\left(\int_{0}^{T}\left\{\eta^{\prime}\left(\bar{u}_{\mathcal{T}, k}\right)-\eta^{\prime}\left(u_{\mathcal{T}, k}\right)\right\} g\left(u_{\mathcal{T}, k}\right) \varphi(x, t) d W(t)\right)^{2}\right] d x \\
& =|B(0, R)| \int_{B(0, R)} \int_{0}^{T} E\left[\left\{\eta^{\prime}\left(\bar{u}_{\mathcal{T}, k}\right)-\eta^{\prime}\left(u_{\mathcal{T}, k}\right)\right\}^{2} g^{2}\left(u_{\mathcal{T}, k}\right) \varphi^{2}(x, t)\right] d t d x \\
& \leqslant|B(0, R)|\|\varphi\|_{\infty}^{2}\left\|\eta^{\prime \prime}\right\|_{\infty}^{2}\|g\|_{\infty}^{2}\left\|\bar{u}_{\mathcal{T}, k}-u_{\mathcal{T}, k}\right\|_{L^{2}(\Omega \times Q)}^{2} \underset{h \rightarrow 0}{\rightarrow} 0,
\end{aligned}
$$

using Proposition 3 . In this way,

$$
E\left[\mathbb{1}_{A}\left(\tilde{C}^{h, k}-C^{h, k}\right)\right] \rightarrow 0 \text { as } h \rightarrow 0 .
$$

II.4 Convergence of $E\left[\mathbb{1}_{A}\left(\tilde{D}^{h, k}-D^{h, k}\right)\right]$

$$
\begin{aligned}
\left|E\left[\mathbb{1}_{A}\left(\tilde{D}^{h, k}-D^{h, k}\right)\right]\right| & =\left|\frac{1}{2} E\left[\sum_{K \in \mathcal{T}_{R}} \sum_{n=0}^{N-1} \int_{K} \int_{n k}^{(n+1) k} \mathbb{1}_{A}\left[\eta^{\prime \prime}\left(u_{K}^{n}\right)-\eta^{\prime \prime}\left(\bar{u}_{\mathcal{T}, k}(t)\right)\right] g^{2}\left(u_{K}^{n}\right) \varphi(x, n k) d x d t\right]\right| \\
& \leqslant \frac{1}{2}\|g\|_{\infty}^{2}\|\varphi\|_{\infty}\left\|\eta^{\prime \prime \prime}\right\|_{\infty}\left\|\bar{u}_{\mathcal{T}, k}-u_{\mathcal{T}, k}\right\|_{L^{1}(\Omega \times B(0, R) \times(0, T))} \\
& \leqslant \frac{c}{2}\|g\|_{\infty}^{2}\|\varphi\|_{\infty}\left\|\eta^{\prime \prime \prime}\right\|_{\infty}\left\|\bar{u}_{\mathcal{T}, k}-u_{\mathcal{T}, k}\right\|_{L^{2}(\Omega \times Q)} \\
& \rightarrow 0 \text { as h } \rightarrow 0 \text { using Proposition } 3
\end{aligned}
$$

In this way,

$$
E\left[\mathbb{1}_{A}\left(\tilde{D}^{h, k}-D^{h, k}\right)\right] \rightarrow 0 \text { as } h \rightarrow 0 .
$$


Conclusion of STEP II:

By gathering the results obtained previously, one gets that for any P-measurable set $A$,

$$
E\left[\mathbb{1}_{A} R^{h, k}\right] \rightarrow 0 \text { as }\left(h, \frac{k}{h}\right) \rightarrow(0,0),
$$

which concludes the proof of the proposition.

Proposition 5 Proposition 4 holds for a general monotone flux $F$, with the same assumptions.

Proof. Most of the proof is exactly the same as the proof of Proposition 4, since we use only the fact that $F$ is a monotone flux, except to show the points I.1 and II.2, where we truly exploit the fact that $F$ was the Godunov numerical flux. In order to adapt these two points of the proof, we use then the decomposition given by Lemma 2 .

$$
F(a, b)=\theta(a, b) F^{G}(a, b)+(1-\theta(a, b)) F_{D}^{L F}(a, b) .
$$

First we have to give a definition of the numerical entropy flux $G$, which uses the above decomposition : for any $a, b \in \mathbb{R}$

$$
G(a, b)=\theta(a, b) G^{G}(a, b)+(1-\theta(a, b)) G_{D}^{L F}(a, b),
$$

where $G^{G}(a, b)=\Phi(s(a, b))$ and $G_{D}^{L F}(a, b)=\frac{\Phi(a)+\Phi(b)}{2}-D(\eta(b)-\eta(a))$.

- In order to show that $\tilde{B}^{h, k}-\bar{B}^{h, k} \geqslant 0$ almost surely, we split the sum into two terms:

$$
\begin{aligned}
& \tilde{B}^{h, k}-\bar{B}^{h, k} \\
& =\sum_{n=0}^{N-1} \sum_{K \in \mathcal{T}_{R}} \frac{k}{|K|} \sum_{\substack{\sigma \in \mathcal{E}_{K} \\
\sigma=\sigma_{K}}} \theta\left(u_{K}^{n}, u_{L}^{n}\right)\left\{v_{K, L}^{n}\left[\eta^{\prime}\left(u_{K}^{n}\right)\left(F^{G}\left(u_{K}^{n}, u_{L}^{n}\right)-f\left(u_{K}^{n}\right)\right)-\left(G^{G}\left(u_{K}^{n}, u_{L}^{n}\right)-\Phi\left(u_{K}^{n}\right)\right)\right]\right. \\
& \left.\quad-v_{L, K}^{n}\left[\eta^{\prime}\left(u_{K}^{n}\right)\left(F^{G}\left(u_{L}^{n}, u_{K}^{n}\right)-f\left(u_{K}^{n}\right)\right)-\left(G^{G}\left(u_{L}^{n}, u_{K}^{n}\right)-\Phi\left(u_{K}^{n}\right)\right)\right]\right\} \int_{K} \varphi(x, n k) d x \\
& +\sum_{n=0}^{N-1} \sum_{K \in \mathcal{T}_{R}} \frac{k}{|K|} \sum_{\substack{\sigma \in \mathcal{E}_{K} \\
\sigma=\sigma_{K, L}}}\left(1-\theta\left(u_{K}^{n}, u_{L}^{n}\right)\right)\left\{v_{K, L}^{n}\left[\eta^{\prime}\left(u_{K}^{n}\right)\left(F_{D}^{L F}\left(u_{K}^{n}, u_{L}^{n}\right)-f\left(u_{K}^{n}\right)\right)-\left(G_{D}^{L F}\left(u_{K}^{n}, u_{L}^{n}\right)-\Phi\left(u_{K}^{n}\right)\right)\right]\right. \\
& \left.\quad-v_{L, K}^{n}\left[\eta^{\prime}\left(u_{K}^{n}\right)\left(F_{D}^{L F}\left(u_{L}^{n}, u_{K}^{n}\right)-f\left(u_{K}^{n}\right)\right)-\left(G_{D}^{L F}\left(u_{L}^{n}, u_{K}^{n}\right)-\Phi\left(u_{K}^{n}\right)\right)\right]\right\} \int_{K} \varphi(x, n k) d x .
\end{aligned}
$$

In order to treat the first sum we just have to apply the same argument as in the proof of the point I.1, whereas the second term can be dealt by using similar argument to the one used in the proof of Proposition 4 of [BCG], since we recall that the modified Lax-Friedrichs scheme belongs to the family of flux-splitting schemes.

- In order to show that for any measurable set $A$ we have $E\left[\mathbb{1}_{A}\left(\bar{B}^{h, k}-B_{1}^{h, k}\right)\right] \underset{h \rightarrow 0}{\rightarrow} 0$, we split once again the sum into two parts:

$$
\begin{array}{r}
\bar{B}^{h, k}-B_{1}^{h, k}=\sum_{n=0}^{N-1} \sum_{K \in \mathcal{T}_{R}} \int_{n k}^{(n+1) k} \frac{1}{|K|} \int_{K} \sum_{\substack{\sigma \in \mathcal{E}_{K} \\
\sigma=\sigma_{K, L}}} \theta\left(u_{K}^{n}, u_{L}^{n}\right)|\sigma|\left\{v_{K, L}^{n} G^{G}\left(u_{K}^{n}, u_{L}^{n}\right)-v_{L, K}^{n} G^{G}\left(u_{L}^{n}, u_{K}^{n}\right)\right\} \varphi(x, n k) d x d t \\
\quad+\sum_{n=0}^{N-1} \sum_{K \in \mathcal{T}_{R}} \int_{n k}^{(n+1) k} \frac{1}{\operatorname{Card}(\mathcal{N}(K))} \int_{K} \sum_{\substack{\sigma \in \mathcal{E}_{K} \\
\sigma=\sigma_{K, L}}} \theta\left(u_{K}^{n}, u_{L}^{n}\right) \Phi\left(u_{K}^{n}\right) \vec{v}(x, t) \cdot \nabla x \varphi(x, n k) d x d t \\
+\sum_{n=0}^{N-1} \sum_{K \in \mathcal{T}_{R}} \int_{n k}^{(n+1) k} \frac{1}{|K|} \int_{K} \sum_{\sigma \in \mathcal{E}_{K}}\left(1-\theta\left(u_{K}^{n}, u_{L}^{n}\right)\right)|\sigma|\left\{v_{K, L}^{n} G_{D}^{L F}\left(u_{K}^{n}, u_{L}^{n}\right)-v_{L, K}^{n} G_{D}^{L F}\left(u_{L}^{n}, u_{K}^{n}\right)\right\} \varphi(x, n k) d x d t \\
+\sum_{n=0}^{N-1} \sum_{K \in \mathcal{T}_{R}} \int_{n k}^{(n+1) k} \frac{1}{\operatorname{Card}(\mathcal{N}(K))} \int_{K} \sum_{\substack{\sigma \in \mathcal{E}_{K} \\
\sigma=\sigma_{K, L}}}\left(1-\theta\left(u_{K}^{n}, u_{L}^{n}\right)\right) \Phi\left(u_{K}^{n}\right) \vec{v}(x, t) \cdot \nabla_{x} \varphi(x, n k) d x d t .
\end{array}
$$

To conclude, we deal with the first two terms by applying the same argument as in the proof of the point II.2, whereas the last two term can be dealt by using similar argument to the one used in the proof of Proposition 4 of BCG].

The following proposition investigates the entropy inequalities which are satisfied by the approximate solution $u_{\mathcal{T}, k}$. 
Proposition 6 (Continuous entropy inequality on the discrete solution) Assume that hypotheses $H_{1}$ to $H_{6}$ hold. Let $\mathcal{T}$ be an admissible mesh in the sense of Definition $3, N \in \mathbb{N}^{\star}$ and let $k=\frac{T}{N} \in \mathbb{R}_{+}^{\star}$ be the time step. Then, P-a.s. in $\Omega$, for any $\eta \in \mathcal{A}$ and for any $\varphi \in \mathcal{D}^{+}\left(\mathbb{R}^{d} \times[0, T)\right)$ :

$$
\begin{aligned}
& \int_{\mathbb{R}^{d}} \eta\left(u_{0}\right) \varphi(x, 0) d x+\int_{Q} \eta\left(u_{\mathcal{T}, k}\right) \varphi_{t}(x, t) d x d t+\int_{Q} \Phi\left(u_{\mathcal{T}, k}\right) \vec{v} \cdot \nabla_{x} \varphi(x, t) d x d t \\
& +\int_{0}^{T} \int_{\mathbb{R}^{d}} \eta^{\prime}\left(u_{\mathcal{T}, k}\right) g\left(u_{\mathcal{T}, k}\right) \varphi(x, t) d x d W(t)+\frac{1}{2} \int_{Q} \eta^{\prime \prime}\left(u_{\mathcal{T}, k}\right) g^{2}\left(u_{\mathcal{T}, k}\right) \varphi(x, t) d x d t \\
\geqslant & \tilde{R}^{h, k}
\end{aligned}
$$

where for any P-measurable set $A, E\left[\mathbb{1}_{A} \tilde{R}^{h, k}\right] \rightarrow 0$ as $\left(h, \frac{k}{h}\right) \rightarrow(0,0)$.

Proof. The proof of this proposition will be separated in two steps: in the first one we will show that inequality 46 holds for a convenient $\tilde{R}^{h, k}$ and in the second step, we will prove that for any P-measurable set $A, E\left[\mathbb{1}_{A} R^{h, k}\right] \rightarrow 0$ as $h \rightarrow 0$.

Let $T>0, u_{0} \in L^{2}\left(\mathbb{R}^{d}\right), \mathcal{T}$ be an admissible mesh in the sense of Definition $3 \quad N \in \mathbb{N}^{\star}$ and $k=\frac{T}{N} \in \mathbb{R}_{+}^{\star}$. We assume that $\left(h, \frac{k}{h}\right) \rightarrow(0,0)$, in this way we can suppose that the CFL Condition

$$
k \leqslant \frac{(1-\xi) \bar{\alpha}^{2} h}{\left(F_{1}+F_{2}\right) V}
$$

holds for some $\xi \in(0,1)$. In this manner, the estimates given by Proposition 1 and Proposition 2 hold. Consider $\eta \in \mathcal{A}$ and $\varphi \in \mathcal{D}^{+}\left(\mathbb{R}^{d} \times[0, T)\right)$, thus there exists $R>h$ such that $\operatorname{supp} \varphi \subset B(0, R-h) \times[0, T[$. We also define $\mathcal{T}_{R}=\{K \in \mathcal{T}$ such that $K \subset B(0, R)\}$.

STEP I: Let us show that inequality 46 holds for a convenient $\tilde{R}^{h, k}$.

Note that the first term of inequality 29 given by Proposition 4 can be rewritten in the following way:

$$
\begin{aligned}
& -\sum_{n=0}^{N-1} \sum_{K \in \mathcal{T}_{R}}\left[\eta\left(u_{K}^{n+1}\right)-\eta\left(u_{K}^{n}\right)\right] \int_{K} \varphi(x, n k) d x \\
= & \int_{k}^{T} \int_{\mathbb{R}^{d}} \eta\left(u_{\mathcal{T}, k}\right) \varphi_{t}(x, t-k) d x d t+\sum_{K \in \mathcal{T}_{R}} \int_{K} \eta\left(u_{K}^{0}\right) \varphi(x, 0) d x .
\end{aligned}
$$

Indeed, thanks to the discrete integration by parts formula

$$
\sum_{n=1}^{N} a_{n}\left(b_{n}-b_{n-1}\right)=a_{N+1} b_{N}-a_{0} b_{0}-\sum_{n=0}^{N} b_{n}\left(a_{n+1}-a_{n}\right)
$$

and by using the fact that for all $x$ in $K$ and for $k$ small enough, $\varphi(x, N k)=\varphi(x,(N-1) k)=0$ we get

$$
\begin{aligned}
\int_{k}^{T} \int_{\mathbb{R}^{d}} \eta\left(u_{\mathcal{T}, k}\right) \varphi_{t}(x, t-k) d x d t= & \sum_{n=1}^{N-1} \sum_{K \in \mathcal{T}_{R}} \int_{K} \eta\left(u_{K}^{n}\right)[\varphi(x, n k)-\varphi(x,(n-1) k)] d x \\
= & -\sum_{n=0}^{N-1} \sum_{K \in \mathcal{T}_{R}} \int_{K}\left[\eta\left(u_{K}^{n+1}\right)-\eta\left(u_{K}^{n}\right)\right] \varphi(x, n k) d x \\
& +\sum_{K \in \mathcal{T}_{R}} \int_{K} \eta\left(u_{K}^{N}\right) \varphi(x,(N-1) k)-\eta\left(u_{K}^{0}\right) \varphi(x, 0) d x \\
= & -\sum_{n=0}^{N-1} \sum_{K \in \mathcal{T}_{R}} \int_{K}\left[\eta\left(u_{K}^{n+1}\right)-\eta\left(u_{K}^{n}\right)\right] \varphi(x, n k) d x \\
& -\sum_{K \in \mathcal{T}_{R}} \int_{K} \eta\left(u_{K}^{0}\right) \varphi(x, 0) d x
\end{aligned}
$$

By denoting

$$
\begin{aligned}
C_{1}^{h, k} & =\int_{0}^{T} \int_{\mathbb{R}^{d}} \eta^{\prime}\left(u_{\mathcal{T}, k}\right) g\left(u_{\mathcal{T}, k}\right) \varphi(x, t) d x d W(t) \\
D_{1}^{h, k} & =\frac{1}{2} \int_{Q} \eta^{\prime \prime}\left(u_{\mathcal{T}, k}\right) g^{2}\left(u_{\mathcal{T}, k}\right) \varphi(x, t) d x d t
\end{aligned}
$$


one gets from inequality 29 , inequality 46 with $\tilde{R}^{h, k}$ defined by

$$
\begin{aligned}
\tilde{R}^{h, k}= & R^{h, k}+\int_{\mathbb{R}^{d}} \eta\left(u_{0}\right) \varphi(x, 0) d x-\sum_{K \in \mathcal{T}_{R}} \int_{K} \eta\left(u_{K}^{0}\right) \varphi(x, 0) d x \\
& +\int_{Q} \eta\left(u_{\mathcal{T}, k}\right) \varphi_{t}(x, t) d x d t-\int_{k}^{T} \int_{\mathbb{R}^{d}} \eta\left(u_{\mathcal{T}, k}\right) \varphi_{t}(x, t-k) d x d t \\
& +\int_{Q} \Phi\left(u_{\mathcal{T}, k}\right) \vec{v} \cdot \nabla_{x} \varphi(x, t) d x d t-\sum_{n=0}^{N-1} \sum_{K \in \mathcal{T}_{R}} \int_{n k}^{(n+1) k} \int_{K} \Phi\left(u_{K}^{n}\right) \vec{v} \cdot \nabla_{x} \varphi(x, n k) d x d t \\
& +C_{1}^{h, k}-\sum_{K \in \mathcal{T}_{R}} \sum_{n=0}^{N-1} \int_{K} \int_{n k}^{(n+1) k} \eta^{\prime}\left(u_{K}^{n}\right) g\left(u_{K}^{n}\right) \varphi(x, n k) d W(t) d x \\
& +D_{1}^{h, k}-\frac{1}{2} \sum_{n=0}^{N-1} \sum_{K \in \mathcal{T}_{R}} \int_{n k}^{(n+1) k} \int_{K} \eta^{\prime \prime}\left(u_{K}^{n}\right) g^{2}\left(u_{K}^{n}\right) \varphi(x, n k) d x d t
\end{aligned}
$$

where $R^{h, k}$ is given by 35 in the proof of the previous proposition.

STEP II: Let us show that for any P-measurable set $A, E\left[\mathbb{1}_{A} \tilde{R}^{h, k}\right] \rightarrow 0$ as $h \rightarrow 0$.

Thanks to Proposition 4 . we know that for any P-measurable set $A, E\left[\mathbb{1}_{A} R^{h, k}\right] \rightarrow 0$ as $h \rightarrow 0$. Then it remains to study the convergence of the following quantities:

$$
\begin{aligned}
& E\left[\mathbb{1}_{A}\left(\int_{\mathbb{R}^{d}} \eta\left(u_{0}\right) \varphi(x, 0) d x-\sum_{K \in \mathcal{T}_{R}} \int_{K} \eta\left(u_{K}^{0}\right) \varphi(x, 0) d x\right)\right], \\
& E\left[\mathbb{1}_{A}\left(\int_{Q} \eta\left(u_{\mathcal{T}, k}\right) \varphi_{t}(x, t) d x d t-\int_{k}^{T} \int_{\mathbb{R}^{d}} \eta\left(u_{\mathcal{T}, k}\right) \varphi_{t}(x, t-k) d x d t\right)\right], \\
& E\left[\mathbb{1}_{A}\left(\int_{Q} \Phi\left(u_{\mathcal{T}, k}\right) \vec{v}(x, t) \cdot \nabla_{x} \varphi(x, t) d x d t-\sum_{n=0}^{N-1} \sum_{K \in \mathcal{T}_{R}} \int_{n k}^{(n+1) k} \int_{K} \Phi\left(u_{K}^{n}\right) \vec{v}(x, t) \cdot \nabla_{x} \varphi(x, n k) d x d t\right)\right], \\
& E\left[\mathbb{1}_{A}\left(C_{1}^{h, k}-\sum_{K \in \mathcal{T}_{R}} \sum_{n=0}^{N-1} \int_{K} \int_{n k}^{(n+1) k} \eta^{\prime}\left(u_{K}^{n}\right) g\left(u_{K}^{n}\right) \varphi(x, n k) d W(t) d x\right)\right]=E\left[\mathbb{1}_{A}\left(C_{1}^{h, k}-\tilde{C}^{h, k}\right)\right] \\
& E\left[\mathbb{1}_{A}\left(D_{1}^{h, k}-\frac{1}{2} \sum_{n=0}^{N-1} \sum_{K \in \mathcal{T}_{R}} \int_{n k}^{(n+1) k} \int_{K} \eta^{\prime \prime}\left(u_{K}^{n}\right) g^{2}\left(u_{K}^{n}\right) \varphi(x, n k) d x d t\right)\right]=E\left[\mathbb{1}_{A}\left(D_{1}^{h, k}-\tilde{D}^{h, k}\right)\right],
\end{aligned}
$$

where $\tilde{C}^{h, k}$ and $\tilde{D}^{h, k}$ have been defined respectively by $(33)$ and 34 in the proof of Proposition 4 . Let us analyze separately the convergence of these terms as $h \rightarrow 0$.

II.1 Convergence of $E\left[\mathbb{1}_{A}\left(\int_{\mathbb{R}^{d}} \eta\left(u_{0}\right) \varphi(x, 0) d x-\sum_{K \in \mathcal{T}_{R}} \int_{K} \eta\left(u_{K}^{0}\right) \varphi(x, 0) d x\right)\right]$

Since $u_{0} \in L_{\text {loc }}^{1}\left(\mathbb{R}^{d}\right)$, one shows that this term tends to 0 as $h \rightarrow 0$.

II.2 Convergence of $E\left[\mathbb{1}_{A}\left(\int_{Q} \eta\left(u_{\mathcal{T}, k}\right) \varphi_{t}(x, t) d x d t-\int_{k}^{T} \int_{\mathbb{R}^{d}} \eta\left(u_{\mathcal{T}, k}\right) \varphi_{t}(x, t-k) d x d t\right)\right]$

$$
\begin{aligned}
& \left|E\left[\int_{Q} \eta\left(u_{\mathcal{T}, k}\right) \varphi_{t}(x, t) d x d t-\int_{k}^{T} \int_{\mathbb{R}^{d}} \eta\left(u_{\mathcal{T}, k}\right) \varphi_{t}(x, t-k) d x d t\right]\right| \\
& \leqslant E\left[\int_{k}^{T} \int_{\mathbb{R}^{d}}\left|\eta\left(u_{\mathcal{T}, k}\right) \| \varphi_{t}(x, t)-\varphi_{t}(x, t-k)\right| d x d t\right]+E\left[\int_{0}^{k} \int_{\mathbb{R}^{d}}\left|\eta\left(u_{\mathcal{T}, k}\right) \| \varphi_{t}(x, t)\right| d x d t\right] \\
& \leqslant\left\|\varphi_{t t}\right\|_{\infty} k\left(\left|\eta(0)\|\operatorname{supp} \varphi \mid+\| \eta^{\prime} \|_{\infty} \sqrt{\left.|\operatorname{supp} \varphi|\left\|u_{\mathcal{T}, k}\right\|_{L^{2}(\Omega \times Q)}\right)}\right.\right. \\
& +k\left\|\varphi_{t}\right\|_{\infty}\left(\left|\eta(0)\|B(0, R) \mid+\| \eta^{\prime}\left\|_{\infty} \sqrt{|B(0, R)|}\right\| u_{\mathcal{T}, k} \|_{L^{\infty}\left(0, T ; L^{2}\left(\Omega \times \mathbb{R}^{d}\right)\right)}\right) .\right.
\end{aligned}
$$

We deduce easily that $E\left[\mathbb{1}_{A}\left(\int_{Q} \eta\left(u_{\mathcal{T}, k}\right) \varphi_{t}(x, t) d x d t-\int_{k}^{T} \int_{\mathbb{R}^{d}} \eta\left(u_{\mathcal{T}, k}\right) \varphi_{t}(x, t-k) d x d t\right)\right]_{h \rightarrow 0}^{\rightarrow} 0$.

II.3 Convergence of $E\left[\mathbb{1}_{A}\left(\int_{Q} \Phi\left(u_{\mathcal{T}, k}\right) \vec{v} . \nabla_{x} \varphi(x, t) d x d t-\sum_{n=0}^{N-1} \sum_{K \in \mathcal{T}_{R}} \int_{n k}^{(n+1) k} \int_{K} \Phi\left(u_{K}^{n}\right) \vec{v}(x, t) . \nabla_{x} \varphi(x, n k) d x d t\right)\right]$ 


$$
\begin{aligned}
& \left|E\left[\mathbb{1}_{A}\left(\int_{Q} \Phi\left(u_{\mathcal{T}, k}\right) \vec{v}(x, t) \cdot \nabla_{x} \varphi(x, t) d x d t-\sum_{n=0}^{N-1} \sum_{K \in \mathcal{T}_{R}} \int_{n k}^{(n+1) k} \int_{K} \Phi\left(u_{K}^{n}\right) \vec{v}(x, t) \cdot \nabla_{x} \varphi(x, n k) d x d t\right)\right]\right| \\
& =\left|\sum_{n=0}^{N-1} \sum_{K \in \mathcal{T}_{R}} E\left[\mathbb{1}_{A} \int_{n k}^{(n+1) k} \int_{K} \Phi\left(u_{K}^{n}\right) \vec{v}(x, t) \cdot\left[\nabla_{x} \varphi(x, t)-\nabla_{x} \varphi(x, n k)\right] d x d t\right]\right| \\
& \leqslant k\left\|\Phi^{\prime}\right\|_{\infty}\left\|\partial_{t} \nabla_{x} \varphi\right\|_{\infty} V E\left[\int_{0}^{T} \int_{B(0, R)}\left|u_{\mathcal{T}, k}\right| d x d t\right] \\
& \leqslant k\left\|\Phi^{\prime}\right\|_{\infty}\left\|\partial_{t} \nabla_{x} \varphi\right\|_{\infty} V \sqrt{T|B(0, R)|}\left\|u_{\mathcal{T}, k}\right\|_{L^{2}(\Omega \times Q)} \\
& \underset{h \rightarrow 0}{h \rightarrow 0}
\end{aligned}
$$

II.4 Convergence of $E\left[\mathbb{1}_{A}\left(C_{1}^{h, k}-\tilde{C}^{h, k}\right)\right]$

Using Cauchy-Schwarz inequality on $\Omega \times B(0, R)$ and Itô isometry one gets

$$
\begin{aligned}
\left|E\left[\mathbb{1}_{A}\left(C_{1}^{h, k}-\tilde{C}^{h, k}\right)\right]\right| & =\left|E\left[\mathbb{1}_{A} \sum_{n=0}^{N-1} \sum_{K \in \mathcal{T}_{R}} \int_{K} \int_{n k}^{(n+1) k} \eta^{\prime}\left(u_{K}^{n}\right) g\left(u_{K}^{n}\right)\{\varphi(x, n k)-\varphi(x, t)\} d W(t) d x\right]\right| \\
& \leqslant \sum_{n=0}^{N-1} \sqrt{|B(0, R)|}\left(\sum_{K \in \mathcal{T}_{R}} \int_{K} E\left[\left(\int_{n k}^{(n+1) k} \eta^{\prime}\left(u_{K}^{n}\right) g\left(u_{K}^{n}\right)\{\varphi(x, n k)-\varphi(x, t)\} d W(t)\right)^{2}\right] d x\right)^{1 / 2} \\
& =\sum_{n=0}^{N-1} \sqrt{|B(0, R)|}\left(\sum_{K \in \mathcal{T}_{R}} \int_{K} \int_{n k}^{(n+1) k} E\left[\left(\eta^{\prime}\left(u_{K}^{n}\right) g\left(u_{K}^{n}\right)\{\varphi(x, n k)-\varphi(x, t)\}\right)^{2}\right] d t d x\right)^{1 / 2} \\
& \leqslant \sqrt{k} \sqrt{|B(0, R)|} C_{g}\left\|\varphi_{t}\right\|_{\infty}\left\|\eta^{\prime}\right\|_{\infty} \sum_{n=0}^{N-1} k\left(\sum_{K \in \mathcal{T}_{R}}|K| E\left[\left(u_{K}^{n}\right)^{2}\right]\right)^{1 / 2} \\
& \leqslant \sqrt{k} \sqrt{|B(0, R)|} C_{g}\left\|\varphi_{t}\right\|_{\infty}\left\|\eta^{\prime}\right\|_{\infty} T e^{T C_{g}^{2} / 2}\left\|u_{0}\right\|_{L^{2}\left(\mathbb{R}^{d}\right)} \rightarrow \begin{array}{c}
\rightarrow \rightarrow 0 \\
h \rightarrow 0
\end{array}
\end{aligned}
$$

where we have used Proposition 1 to conclude.

II.5 Convergence of $E\left[\mathbb{1}_{A}\left(D_{1}^{h, k}-\tilde{D}^{h, k}\right)\right]$

$$
\begin{aligned}
\left|E\left[\mathbb{1}_{A}\left(D_{1}^{h, k}-\tilde{D}^{h, k}\right)\right]\right| & =\left|\frac{1}{2} E\left[\sum_{n=0}^{N-1} \sum_{K \in \mathcal{T}_{R}} \int_{n k}^{(n+1) k} \int_{K} \mathbb{1}_{A} \eta^{\prime \prime}\left(u_{K}^{n}\right) g^{2}\left(u_{K}^{n}\right)[\varphi(x, n k)-\varphi(x, t)] d x d t\right]\right| \\
& \leqslant \frac{1}{2} k\left\|\eta^{\prime \prime}\right\|_{\infty}\|g\|_{\infty}^{2}\left\|\varphi_{t}\right\|_{\infty} T|B(0, R)| \underset{h \rightarrow 0}{\rightarrow} 0 .
\end{aligned}
$$

To summarize, we proved in this second step that $E\left[\mathbb{1}_{A} \tilde{R}^{h, k}\right] \rightarrow 0$ as $h \rightarrow 0$, which concludes the proof of the proposition.

\subsection{Proof of the convergence}

And we prove now the convergence of the finite volume approximation $u_{\mathcal{T}, k}$ to the stochastic entropy solution of Problem (1).

Theorem 3 (Convergence to the stochastic entropy solution) Assume that hypotheses $H_{1}$ to $H_{6}$ hold. Let $\mathcal{T}$ be an admissible mesh in the sense of Definition $3, N \in \mathbb{N}^{*}$, let $k=\frac{T}{N} \in \mathbb{R}_{+}^{*}$ be the time step. Let $u_{\mathcal{T}, k}$ be the finite volume approximation defined by (6). Then $u_{\mathcal{T}, k}$ converges in $L_{l o c}^{p}(\Omega \times Q)$ for any $1 \leqslant p<2$ to the unique stochastic entropy solution of (1) in the sense of Definition 1 as $h \rightarrow 0$ with $\frac{k}{h} \rightarrow 0$. We recall that $L_{\text {loc }}^{p}(\Omega \times Q)$ means locally in space.

Proof. Let $\mathcal{T}$ be an admissible mesh in the sense of Definition $3 \quad N \in \mathbb{N}^{\star}$ and let $k=\frac{T}{N} \in \mathbb{R}_{+}^{\star}$ be the time step such that $k / h \rightarrow 0$ as $h \rightarrow 0$. In this way we can suppose that the CFL Condition

$$
k \leqslant \frac{(1-\xi) \bar{\alpha}^{2} h}{\left(F_{1}+F_{2}\right) V},
$$

holds for some $\xi \in(0,1)$. In this manner, the estimates given by Proposition 1 and Proposition 2 hold. Consider $A$ a P-measurable set, $\eta \in \mathcal{A}, \varphi \in \mathcal{D}^{+}\left(\mathbb{R}^{d} \times[0, T)\right)$, thus there exists $R>h$ such that $\operatorname{supp} \varphi \subset$ 
$B(0, R-h) \times[0, T)$. We also define $\mathcal{T}_{R}=\{K \in \mathcal{T}$ such that $K \subset B(0, R)\}$.

Let us multiply inequality 46 by $\mathbb{1}_{A}$ and take the expectation. This yields:

$$
\begin{aligned}
& E\left[\mathbb{1}_{A} \int_{\mathbb{R}^{d}} \eta\left(u_{0}\right) \varphi(x, 0) d x\right]+E\left[\mathbb{1}_{A} \int_{Q} \eta\left(u_{\mathcal{T}, k}\right) \varphi_{t}(x, t) d x d t\right]+E\left[\mathbb{1}_{A} \int_{Q} \Phi\left(u_{\mathcal{T}, k}\right) \vec{v}(x, t) \cdot \nabla_{x} \varphi(x, t) d x d t\right] \\
& +E\left[\mathbb{1}_{A} \int_{0}^{T} \int_{\mathbb{R}^{d}} \eta^{\prime}\left(u_{\mathcal{T}, k}\right) g\left(u_{\mathcal{T}, k}\right) \varphi(x, t) d x d W(t)\right]+\frac{1}{2} E\left[\mathbb{1}_{A} \int_{Q} \eta^{\prime \prime}\left(u_{\mathcal{T}, k}\right) g^{2}\left(u_{\mathcal{T}, k}\right) \varphi(x, t) d x d t\right] \\
\geqslant & E\left[\mathbb{1}_{A} \tilde{R}^{h, k}\right] .
\end{aligned}
$$

To show the convergence of $u_{\mathcal{T}, k}$ towards the unique stochastic entropy solution of our problem, we aim to pass to the limit in the above inequality. Thanks to Proposition 6 we know that for any P-measurable set $A, E\left[\mathbb{1}_{A} \tilde{R}^{h, k}\right] \rightarrow 0$ as $h \rightarrow 0$. Thus it remains to study the convergence of the left-hand side of 47 ). Recall that thanks to the a priori estimate stated in Proposition 1, $u_{\mathcal{T}, k}$ converges (up to a subsequence denoted in the same way) in the sense of Young measures to an "entropy process" denoted by $\mathbf{u}$ in $L^{2}(\Omega \times Q \times(0,1))$ (see Section 4.3).

1. Study of $E\left[\mathbb{1}_{A} \int_{Q} \eta\left(u_{\mathcal{T}, k}\right) \varphi_{t}(x, t) d x d t\right]$

Note that $\Psi:(\omega, x, t, \nu) \in \Omega \times Q \times \mathbb{R} \mapsto \mathbb{1}_{A}(\omega) \eta(\nu) \varphi_{t}(x, t) \in \mathbb{R}$ is a Carathéodory function such that $\Psi\left(., u_{\mathcal{T}, k}\right)$ is bounded in $L^{2}(\Omega \times Q)$, using the compact support of $\varphi$ it is therefore uniformly integrable, thus

$$
E\left[\mathbb{1}_{A} \int_{Q} \eta\left(u_{\mathcal{T}, k}(x, t)\right) \varphi_{t}(x, t) d x d t\right] \rightarrow E\left[\mathbb{1}_{A} \int_{Q} \int_{0}^{1} \eta(\mathbf{u}(x, t, \alpha)) d \alpha \varphi_{t}(x, t) d x d t\right] \text { as } h \rightarrow 0
$$

2. Study of $E\left[\mathbb{1}_{A} \int_{Q} \Phi\left(u_{\mathcal{T}, k}\right) \vec{v}(x, t) \cdot \nabla_{x} \varphi(x, t) d x d t\right]$

Since $\Phi\left(u_{\mathcal{T}, k}\right)$ is bounded in $L^{2}(\Omega \times Q)$, using the same arguments as previously, we obtain

$$
E\left[\mathbb{1}_{A} \int_{Q} \Phi\left(u_{\mathcal{T}, k}\right) \vec{v}(x, t) \cdot \nabla_{x} \varphi(x, t) d x d t\right] \rightarrow E\left[\mathbb{1}_{A} \int_{Q} \int_{0}^{1} \Phi(\mathbf{u}(x, t, \alpha)) \vec{v}(x, t) \cdot \nabla_{x} \varphi(x, t) d \alpha d x d t\right] \text { as } h \rightarrow 0
$$

3. Study of $E\left[\mathbb{1}_{A} \int_{0}^{T} \int_{\mathbb{R}^{d}} \eta^{\prime}\left(u_{\mathcal{T}, k}\right) g\left(u_{\mathcal{T}, k}\right) \varphi(x, t) d x d W(t)\right]$

By denoting $\Psi:(\omega, x, t, \nu) \in \Omega \times Q \times \mathbb{R} \mapsto \eta^{\prime}(\nu) g(\nu) \varphi(x, t) \in \mathbb{R}$, thanks to Proposition 1. $\Psi\left(., u_{\mathcal{T}, k}\right)$ is bounded in $L^{2}(\Omega \times Q)$, and therefore $\Psi\left(., u_{\mathcal{T}, k}\right)$ converges weakly (up to a subsequence denoted in the same way) in $L^{2}(\Omega \times Q)$ to an element called $\chi$.

But, for any $\phi \in L^{2}(\Omega \times Q),(\omega, x, t, \nu) \in \Omega \times Q \times \mathbb{R} \mapsto \phi(\omega, x, t) \Psi(\omega, x, t, \nu)$ is a Carathéodory function such that $\left(\phi \Psi\left(., u_{\mathcal{T}, k}\right)\right)$ is uniformly integrable. It is based on the fact that for any subset $H$ of $\Omega \times Q$,

$$
\int_{H}\left|\phi \Psi\left(., u_{\mathcal{T}, k}\right)\right| d x d t d P \leqslant\left\|\Psi\left(., u_{\mathcal{T}, k}\right)\right\|_{L^{2}(H)}\left[\int_{H}|\phi|^{2} d x d t d P\right]^{1 / 2} .
$$

Thus, at the limit,

$$
\int_{\Omega \times Q} \chi \phi d x d t d P=\int_{\Omega \times Q} \int_{0}^{1} \Psi(., \mathbf{u}(., \alpha)) d \alpha \phi d x d t d P .
$$

By identification, $\Psi\left(., u_{\mathcal{T}, k}\right) \rightarrow \int_{0}^{1} \Psi(., \mathbf{u}(., \alpha)) d \alpha$ weakly in $L^{2}(\Omega \times Q)$. Using now the linear continuity of the stochastic integral from $L^{2}(\Omega \times Q)$ to $L^{2}\left(\Omega \times \mathbb{R}^{d}\right)$, which implies the continuity for the weak topology:

$$
\int_{0}^{T} \eta^{\prime}\left(u_{\mathcal{T}, k}\right) g\left(u_{\mathcal{T}, k}\right) \varphi d W(t) \rightarrow \int_{0}^{T} \int_{0}^{1} \eta^{\prime}(\mathbf{u}(., \alpha)) g(\mathbf{u}(., \alpha)) \varphi d \alpha d W(t) \text { weakly in } L^{2}\left(\Omega \times \mathbb{R}^{d}\right)
$$

As $\mathbb{1}_{A} \mathbb{1}_{B(0, R)} \in L^{2}\left(\Omega \times \mathbb{R}^{d}\right)$ one gets at the limit

$E\left[\mathbb{1}_{A} \int_{0}^{T} \int_{\mathbb{R}^{d}} \eta^{\prime}\left(u_{\mathcal{T}, k}\right) g\left(u_{\mathcal{T}, k}\right) \varphi(x, t) d x d W(t)\right] \rightarrow E\left[\mathbb{1}_{A} \int_{0}^{T} \int_{\mathbb{R}^{d}} \int_{0}^{1} \eta^{\prime}(\mathbf{u}(x, t, \alpha)) g(\mathbf{u}(x, t, \alpha)) \varphi(x, t) d \alpha d x d W(t)\right]$.

4. Study of $\frac{1}{2} E\left[\mathbb{1}_{A} \int_{Q} \eta^{\prime \prime}\left(u_{\mathcal{T}, k}\right) g^{2}\left(u_{\mathcal{T}, k}\right) \varphi(x, t) d x d t\right]$

Since $\Psi:(\omega, x, t, \nu) \in \Omega \times Q \times \mathbb{R} \mapsto \eta^{\prime \prime}(\nu) g^{2}(\nu) \varphi(x, t) \mathbb{1}_{A}(\omega) \in \mathbb{R}$ is a Carathéodory function such that $\Psi\left(., u_{\mathcal{T}, k}\right)$ is bounded in $L^{2}(\Omega \times Q)$, at the limit we get:

$$
\frac{1}{2} E\left[\mathbb{1}_{A} \int_{Q} \eta^{\prime \prime}\left(u_{\mathcal{T}, k}\right) g^{2}\left(u_{\mathcal{T}, k}\right) \varphi(x, t) d x d t\right] \rightarrow \frac{1}{2} E\left[1_{A} \int_{Q} \int_{0}^{1} \eta^{\prime \prime}(\mathbf{u}(x, t, \alpha)) g^{2}(\mathbf{u}(x, t, \alpha)) \varphi(x, t) d \alpha d x d t\right]
$$


Finally, by passing to the limit in inequality 47 , we obtain:

For any P-measurable set $A$, for any $\eta \in \mathcal{A}$ and for any $\varphi \in \mathcal{D}^{+}\left(\mathbb{R}^{d} \times[0, T)\right)$

$$
\begin{aligned}
0 \leqslant & E\left[\mathbb{1}_{A} \int_{\mathbb{R}^{d}} \eta\left(u_{0}\right) \varphi(x, 0) d x\right]+E\left[\mathbb{1}_{A} \int_{Q} \int_{0}^{1} \eta(\mathbf{u}(x, t, \alpha)) \varphi_{t}(x, t) d \alpha d x d t\right] \\
& +E\left[\mathbb{1}_{A} \int_{Q} \int_{0}^{1} \Phi(\mathbf{u}(x, t, \alpha)) \vec{v}(x, t) \cdot \nabla_{x} \varphi(x, t) d \alpha d x d t\right] \\
& +E\left[\mathbb{1}_{A} \int_{0}^{T} \int_{\mathbb{R}^{d}} \int_{0}^{1} \eta^{\prime}(\mathbf{u}(x, t, \alpha)) g(\mathbf{u}(x, t, \alpha)) \varphi(x, t) d \alpha d x d W(t)\right] \\
& +E\left[\mathbb{1}_{A} \frac{1}{2} \int_{Q} \int_{0}^{1} \eta^{\prime \prime}(\mathbf{u}(x, t, \alpha)) g^{2}(\mathbf{u}(x, t, \alpha)) \varphi(x, t) d \alpha d x d t\right] .
\end{aligned}
$$

Hence $\mathbf{u}$ is a measure-valued entropy solution in the sense of Definition 2, Thanks to Theorem 1 $\mathbf{u}$ is independent of $\alpha$ and is hence the unique stochastic entropy solution in the sense of Definition 1 and we denote it by $u$. Hence, all the sequence of approximate solution $u_{\mathcal{T}, k}$ converges to $u$ in $L_{l o c}^{1}(\Omega \times Q)$. In addition, since $u_{\mathcal{T}, k}$ is bounded in $L^{2}(\Omega \times Q)$, all the sequence converges in $L_{l o c}^{p}(\Omega \times Q)$ for any $1 \leqslant p<2$.

\section{A Theoretical background}

The aim of this appendix is to prove the well posedness result stated in Theorem 1 The existence of a solution is based on a parabolic regularization of our stochastic conservation law (1). The proof of existence and uniqueness of the associated viscous solution (denoted $u_{\epsilon}$ in the sequel) is a classic one but for the sake of completness we propose to redevelop the proof in Section A.1 In Section A.2.1. existence of a measurevalued entropy solution in the sense of Definition 2 is proved by passing to the limit on the viscosity parameter (denoted $\epsilon$ ), using as previously convergence in the sense of Young measures. Section A.2.2 is then devoted to the proof of uniqueness of such a solution and as a by-product we deduce the existence and uniqueness of the entropy solution of Problem (1) in the sense of Definition 1. Note that the following proofs are adapted from the work of BAuzet-Vallet-Wittbold [BVW12] to the case of a time-space dependent flux-function.

Remark 11 The existence result follows from the convergence of the finite volume approximation to the solution of (1). However, in order to prove the uniqueness result, we need to know that the solution of (1) is the limit of the solution of the parabolic regularization (48).

\section{A.1 On the parabolic regularization}

We are interested in this section in a viscous regularization of Problem (1) given by the following formal stochastic PDE of nonlinear parabolic type for any $\epsilon>0$ :

$$
\left\{\begin{aligned}
d u_{\epsilon}-\epsilon \Delta u_{\epsilon} d t+\operatorname{div}\left[\vec{v}(x, t) f\left(u_{\epsilon}\right)\right] d t & =g\left(u_{\epsilon}\right) d W \quad \text { in } \Omega \times \mathbb{R}^{d} \times(0, T), \\
u_{\epsilon}(\omega, x, 0) & =u_{0}^{\epsilon}(x), \omega \in \Omega, x \in \mathbb{R}^{d} .
\end{aligned}\right.
$$

Proposition 7 Set $u_{0}^{\epsilon}$ in $H_{0}^{1}\left(\mathbb{R}^{d}\right)$. Then, for any positive $\epsilon$, there exists a unique process $u_{\epsilon} \in \mathcal{N}_{w}^{2}\left(0, T ; H_{0}^{1}\left(\mathbb{R}^{d}\right)\right) \cap$ $\mathcal{C}\left([0, T] ; L^{2}\left(\Omega \times \mathbb{R}^{d}\right)\right)$ weak solution of Problem 48 such that $\partial_{t}\left[u_{\epsilon}-\int_{0}^{t} g\left(u_{\epsilon}\right) d W\right]$ and $\Delta u_{\epsilon}$ are elements of $L^{2}(\Omega \times Q)$. Moreover, there exists a positive constant $C$ such that

$$
\forall \epsilon>0, \quad\left\|u_{\epsilon}\right\|_{L^{\infty}\left(0, T ; L^{2}\left(\Omega \times \mathbb{R}^{d}\right)\right)}^{2}+\epsilon\left\|u_{\epsilon}\right\|_{L^{2}\left((0, T) \times \Omega ; H_{0}^{1}\left(\mathbb{R}^{d}\right)\right)}^{2} \leqslant C .
$$

Proof. (of Proposition 7) Following Val08, we propose a result of existence of a solution based on an implicit time discretization. Let us first introduce some classical notations needed in the sequel.

Definition 5 For any sequence $\left(x_{n}\right) \subset X$, where $X$ is any Banach space, let us denote by

$$
\begin{aligned}
x^{\Delta t} & =\sum_{k=1}^{N} x_{k} \mathbb{1}_{[(k-1) \Delta t, k \Delta t)}, \\
\widetilde{x}^{\Delta t} & =\sum_{k=1}^{N}\left[\frac{x_{k}-x_{k-1}}{\Delta t}[t-(k-1) \Delta t]+x_{k-1}\right] \mathbb{1}_{[(k-1) \Delta t, k \Delta t)}, \\
\text { Thus, } \frac{\partial \widetilde{x}^{\Delta t}}{\partial t} & =\sum_{k=1}^{N} \frac{x_{k}-x_{k-1}}{\Delta t} \mathbb{1}_{[(k-1) \Delta t, k \Delta t)},
\end{aligned}
$$


and elementary calculus yields

$$
\begin{aligned}
& \left\|x^{\Delta t}\right\|_{L^{2}(0, T ; X)}^{2}=\Delta t \sum_{k=1}^{N}\left\|x_{k}\right\|_{X}^{2} ; \quad\left\|\widetilde{x}^{\Delta t}\right\|_{L^{2}(0, T ; X)}^{2} \leqslant \Delta t \sum_{k=0}^{N}\left\|x_{k}\right\|_{X}^{2} ; \\
& \left\|x^{\Delta t}-\widetilde{x}^{\Delta t}\right\|_{L^{2}(0, T ; X)}^{2}=\Delta t \sum_{k=0}^{N-1}\left\|x_{k+1}-x_{k}\right\|_{X}^{2} ; \\
& \left\|\frac{\partial \widetilde{x}^{\Delta t}}{\partial t}\right\|_{L^{2}(0, T ; X)}^{2}=\frac{1}{\Delta t} \sum_{k=0}^{N-1}\left\|x_{k+1}-x_{k}\right\|_{X}^{2} ; \\
& \left\|x^{\Delta t}\right\|_{L^{\infty}(0, T ; X)}=\max _{k=1, \ldots, N}\left\|x_{k}\right\|_{X} ; \quad ; \quad\left\|\widetilde{x}^{\Delta t}\right\|_{L^{\infty}(0, T ; X)}=\max _{k=0, \ldots, N}\left\|x_{k}\right\|_{X} .
\end{aligned}
$$

Then the implicit scheme is the following one:

For given small positive parameter $\Delta t$ and $u_{n}$ in $L^{2}\left(\Omega, H_{0}^{1}\left(\mathbb{R}^{d}\right)\right), \mathcal{F}_{n \Delta t}$-measurable, find $u_{n+1}$ in $L^{2}\left(\Omega, H_{0}^{1}\left(\mathbb{R}^{d}\right)\right)$, $\mathcal{F}_{(n+1) \Delta t}$-measurable, such that $P$-a.s and for any $v$ in $H_{0}^{1}\left(\mathbb{R}^{d}\right)$

$$
\int_{\mathbb{R}^{d}}\left[\left(u_{n+1}-u_{n}\right) v+\Delta t\left\{\epsilon \nabla u_{n+1} \cdot \nabla v-\vec{v}(x, n \Delta t) f\left(u_{n+1}\right) \cdot \nabla v\right\}\right] d x=\left(W_{n+1}-W_{n}\right) \int_{\mathbb{R}^{d}} g\left(u_{n}\right) v d x,
$$

where $W_{n}=W(n \Delta t)$.

Lemma 3 If $\Delta t<\frac{2 \epsilon}{\left(V\left\|f^{\prime}\right\|_{\infty}\right)^{2}}$, such a sequence $\left(u_{n}\right)$ exists.

Proof. (of Lemma 3)

Denote by $\mathbb{V}=L^{2}\left(\Omega, H^{1}\left(\mathbb{R}^{d}\right), \mathcal{F}_{(n+1) \Delta t}, P\right), \mathbb{H}=L^{2}\left(\Omega, L^{2}\left(\mathbb{R}^{d}\right), \mathcal{F}_{(n+1) \Delta t}, P\right)$ and by $T$ the application, defined for any $S \in \mathbb{H}$, by $u=T(S)$ is the solution in $\mathbb{V}$ of the variational problem

$$
\forall v \in \mathbb{V}, E\left[\int_{\mathbb{R}^{d}}\left[\left(u-u_{n}\right) v+\Delta t\{\epsilon \nabla u \cdot \nabla v-\vec{v}(x, n \Delta t) f(S) . \nabla v\}\right] d x\right]=E\left[\left(W_{n+1}-W_{n}\right) \int_{\mathbb{R}^{d}} g\left(u_{n}\right) v d x\right] .
$$

Thanks to the theorem of Lax-Milgram, $T$ is a well-defined function. Moreover, for any $S_{1}, S_{2} \in \mathbb{H}$, one has that

$$
E\left[\int_{\mathbb{R}^{d}}\left|u_{1}-u_{2}\right|^{2} d x+\Delta t \epsilon E \int_{\mathbb{R}^{d}}\left|\nabla\left(u_{1}-u_{2}\right)\right|^{2} d x\right]=\Delta t E\left[\int_{\mathbb{R}^{d}} \vec{v}(x, n \Delta t)\left(f\left(S_{1}\right)-f\left(S_{2}\right)\right) \cdot \nabla\left(u_{1}-u_{2}\right) d x\right],
$$

and

$E\left[\int_{\mathbb{R}^{d}}\left|T\left(S_{1}\right)-T\left(S_{2}\right)\right|^{2} d x\right]+\frac{\Delta t}{2} \epsilon E\left[\int_{\mathbb{R}^{d}}\left|\nabla\left(T\left(S_{1}\right)-T\left(S_{2}\right)\right)\right|^{2} d x\right] \leqslant \frac{\Delta t}{2 \epsilon} E\left[\int_{\mathbb{R}^{d}}(\vec{v}(x, n \Delta t))^{2}\left(f\left(S_{1}\right)-f\left(S_{2}\right)\right)^{2} d x\right]$.

Thus, if $\Delta t<\frac{2 \epsilon}{\left(V\left\|f^{\prime}\right\|_{\infty}\right)^{2}}$ (where $V$ is given by hypothesis $\left.\left[\mathrm{H}_{5}\right]\right), T$ is a contractive mapping in $\mathbb{H}$ and the result holds.

Setting the test-function $u_{n+1}$ in $49 p$ and using the formula $a b=\frac{1}{2}\left[a^{2}+b^{2}-(a-b)^{2}\right]$ with $a=u_{n+1}-u_{n}$ and $b=u_{n+1}$ yields

$$
\begin{aligned}
& \frac{1}{2} E\left[\int_{\mathbb{R}^{d}}\left[\left|u_{n+1}\right|^{2}-\left|u_{n}\right|^{2}+\left|u_{n+1}-u_{n}\right|^{2}\right] d x\right]+\Delta t \epsilon E\left[\int_{\mathbb{R}^{d}}\left|\nabla u_{n+1}\right|^{2} d x\right]-\Delta t E\left[\int_{\mathbb{R}^{d}} \vec{v}(x, n \Delta t) f\left(u_{n+1}\right) \cdot \nabla u_{n+1} d x\right] \\
= & E\left[\left(W_{n+1}-W_{n}\right) \int_{\mathbb{R}^{d}} g\left(u_{n}\right)\left[u_{n+1}-u_{n}\right] d x\right]+E\left[\left(W_{n+1}-W_{n}\right) \int_{\mathbb{R}^{d}} g\left(u_{n}\right) u_{n} d x\right] .
\end{aligned}
$$

Note that since $\operatorname{div}[\vec{v}(x, t)]=0 \forall(x, t) \in \mathbb{R}^{d} \times[0, T], \int_{\mathbb{R}^{d}} \vec{v}(x, t) f(u) \cdot \nabla u d x=0$ for any $t$ in $[0, T]$ and any $u \in D\left(\mathbb{R}^{d}\right)$, thus for any $u \in H^{1}\left(\mathbb{R}^{d}\right)$. Then

$$
\begin{aligned}
& \frac{1}{2} E\left[\int_{\mathbb{R}^{d}}\left[\left|u_{n+1}\right|^{2}-\left|u_{n}\right|^{2}+\left|u_{n+1}-u_{n}\right|^{2}\right] d x\right]+\Delta t \epsilon E\left[\int_{\mathbb{R}^{d}}\left|\nabla u_{n+1}\right|^{2} d x\right] \\
\leqslant & \Delta t E\left[\int_{\mathbb{R}^{d}} g^{2}\left(u_{n}\right) d x\right]+\frac{1}{4} E\left[\int_{\mathbb{R}^{d}}\left[u_{n+1}-u_{n}\right]^{2} d x\right],
\end{aligned}
$$

and, if one denotes by $\|\cdot\|$ the norm in $L^{2}\left(\mathbb{R}^{d}\right)$

$$
\frac{1}{2} E\left[\left\|u_{n}\right\|^{2}\right]+\frac{1}{4} \sum_{k=0}^{n-1} E\left[\left\|u_{k+1}-u_{k}\right\|^{2}\right]+\Delta t \epsilon \sum_{k=0}^{n-1} E\left[\left\|\nabla u_{k+1}\right\|^{2}\right] \leqslant \frac{1}{2}\left\|u_{0}\right\|^{2}+\Delta t \sum_{k=0}^{n-1} E\left[\left\|g\left(u_{k}\right)\right\|^{2}\right],
$$

The discrete Gronwall lemma asserts then that

$$
\begin{aligned}
\frac{1}{2} E\left[\left\|u_{n}\right\|^{2}\right]+\frac{1}{4} \sum_{k=0}^{n-1} E\left[\left\|u_{k+1}-u_{k}\right\|^{2}\right]+\Delta t \epsilon \sum_{k=0}^{n-1} E\left[\left\|\nabla u_{k+1}\right\|^{2}\right] & \leqslant \frac{1}{2}\left\|u_{0}\right\|^{2}+\left\|u_{0}\right\|^{2} \Delta t\left\|g^{\prime}\right\|_{\infty}^{2} \sum_{k=0}^{n-1} e^{2\left\|g^{\prime}\right\|_{\infty}^{2} k \Delta t} \\
& \leqslant C
\end{aligned}
$$


Using the notations of Definition 5 this only gives an $L^{\infty}\left(0, T, L^{2}\left(\Omega \times \mathbb{R}^{d}\right)\right)$ estimate on $u^{\Delta t}$ and an $L^{2}(\Omega \times Q)$ estimate on $\epsilon \nabla u^{\Delta t}$.

Since $u_{0} \in H^{1}\left(\mathbb{R}^{d}\right)$, setting the test-function $v=u_{n+1}-u_{n}-\left(W_{n+1}-W_{n}\right) g\left(u_{n}\right)$ in 49 yields

$$
\begin{aligned}
& \left\|u_{n+1}-u_{n}-\left(W_{n+1}-W_{n}\right) g\left(u_{n}\right)\right\|_{L^{2}\left(\mathbb{R}^{d}\right)}^{2} \\
& +\Delta t \epsilon \int_{\mathbb{R}^{d}} \nabla u_{n+1} \cdot \nabla\left[u_{n+1}-u_{n}-\left(W_{n+1}-W_{n}\right) g\left(u_{n}\right)\right] d x \\
= & \Delta t \int_{\mathbb{R}^{d}}\left[u_{n+1}-u_{n}-\left(W_{n+1}-W_{n}\right) g\left(u_{n}\right)\right] \vec{v}(x, n \Delta t) f\left(u_{n+1}\right) \cdot \nabla u_{n+1} d x \\
\leqslant & \frac{1}{2}\left\|u_{n+1}-u_{n}-\left(W_{n+1}-W_{n}\right) g\left(u_{n}\right)\right\|_{L^{2}\left(\mathbb{R}^{d}\right)}^{2}+\frac{1}{2} C\left(f^{\prime}, V\right)(\Delta t)^{2}\left\|\nabla u_{n+1}\right\|_{L^{2}\left(\mathbb{R}^{d}\right)^{d} .}^{2} .
\end{aligned}
$$

Since $E\left[\left(W_{n+1}-W_{n}\right) \int_{\mathbb{R}^{d}} \nabla u_{n} \cdot \nabla g\left(u_{n}\right) d x\right]=0$, one gets that

$$
\begin{aligned}
& E\left[\int_{\mathbb{R}^{d}} \nabla u_{n+1} \cdot \nabla\left[u_{n+1}-u_{n}-\left(W_{n+1}-W_{n}\right) g\left(u_{n}\right)\right] d x\right] \\
= & \frac{1}{2} E\left[\left\|\nabla u_{n+1}\right\|_{L^{2}\left(\mathbb{R}^{d}\right)^{d}}^{2}+\left\|\nabla\left(u_{n+1}-u_{n}\right)\right\|_{L^{2}\left(\mathbb{R}^{d}\right)^{d}}^{2}-\left\|\nabla u_{n}\right\|_{L^{2}\left(\mathbb{R}^{d}\right)^{d}}^{2}\right] \\
& -E\left[\left(W_{n+1}-W_{n}\right) \int_{\mathbb{R}^{d}} \nabla\left[u_{n+1}-u_{n}\right] \cdot \nabla g\left(u_{n}\right) d x\right] \\
\geq & \frac{1}{2} E\left[\left\|\nabla u_{n+1}\right\|_{L^{2}\left(\mathbb{R}^{d}\right)^{d}}^{2}+\frac{1}{2}\left\|\nabla\left(u_{n+1}-u_{n}\right)\right\|_{L^{2}\left(\mathbb{R}^{d}\right)^{d}}^{2}-\left\|\nabla u_{n}\right\|_{L^{2}\left(\mathbb{R}^{d}\right)^{d}}^{2}-2 \Delta t\left\|\nabla g\left(u_{n}\right)\right\|_{L^{2}\left(\mathbb{R}^{d}\right)^{d}}^{2}\right] .
\end{aligned}
$$

And then

$$
\begin{aligned}
& E\left[\left\|u_{n+1}-u_{n}-\left(W_{n+1}-W_{n}\right) g\left(u_{n}\right)\right\|_{L^{2}\left(\mathbb{R}^{d}\right)}^{2}\right] \\
& +\Delta t \epsilon E\left[\left\|\nabla u_{n+1}\right\|_{L^{2}\left(\mathbb{R}^{d}\right)^{d}}^{2}-\left\|\nabla u_{n}\right\|_{L^{2}\left(\mathbb{R}^{d}\right)^{d}}^{2}+\frac{1}{2}\left\|\nabla\left(u_{n+1}-u_{n}\right)\right\|_{L^{2}\left(\mathbb{R}^{d}\right)^{d}}^{2}\right] \\
\leqslant & 2(\Delta t)^{2} \epsilon E\left[\left\|\nabla g\left(u_{n}\right)\right\|_{L^{2}\left(\mathbb{R}^{d}\right)^{d}}^{2}\right]+C\left(V, f^{\prime}\right)(\Delta t)^{2} E\left[\left\|\nabla u_{n+1}\right\|_{L^{2}\left(\mathbb{R}^{d}\right)^{d}}^{2}\right] .
\end{aligned}
$$

Consequently, for any $k$,

$$
\begin{aligned}
& \sum_{n=0}^{k} \Delta t E\left[\left\|\frac{u_{n+1}-u_{n}-\left(W_{n+1}-W_{n}\right) g\left(u_{n}\right)}{\Delta t}\right\|^{2}\right]+\epsilon E\left[\left\|\nabla u_{n+1}\right\|_{L^{2}\left(\mathbb{R}^{d}\right)^{d}}^{2}\right]+\frac{\epsilon}{2} \sum_{n=0}^{k} E\left[\left\|\nabla\left(u_{n+1}-u_{n}\right)\right\|_{L^{2}\left(\mathbb{R}^{d}\right)^{d}}^{2}\right] \\
\leqslant & C\left(V, f^{\prime}, g^{\prime}\right) \Delta t \sum_{n=0}^{k+1} E\left[\left\|\nabla u_{n}\right\|_{L^{2}\left(\mathbb{R}^{d}\right)^{d}}^{2}\right]+\epsilon E\left[\left\|\nabla u_{0}\right\|_{L^{2}\left(\mathbb{R}^{d}\right)^{d}}^{2}\right] \leqslant C t e .
\end{aligned}
$$

Let us define for any $(x, t) \in \mathbb{R}^{d} \times[0, T], \vec{v}^{\Delta t}(x, t)=\sum_{k=1}^{N} \vec{v}(x, k \Delta t) \mathbb{1}_{[(k-1) \Delta t, k \Delta t)}(t)$ and denote

$\widetilde{B}^{\Delta t}=\sum_{k=1}^{N}\left[\frac{B_{k}-B_{k-1}}{\Delta t}[t-(k-1) \Delta t]+B_{k-1}\right] \mathbb{1}_{[(k-1) \Delta t, k \Delta t)}$

with $B_{n}=\sum_{k=0}^{n-1}\left(W^{k+1}-W^{k}\right) g\left(u^{k}\right)=\int_{0}^{n \Delta t} g\left(u^{\Delta t}(.-\Delta t)\right) d W *$

Thanks to $\left[52\right.$, one gets that $u^{\Delta t}$ and $\widetilde{u}^{\Delta t}$ are bounded in $L^{\infty}\left(0, T, L^{2}\left(\Omega, H^{1}\left(\mathbb{R}^{d}\right)\right)\right)$, that $\partial_{t}\left[\widetilde{u}^{\Delta t}-\widetilde{B}^{\Delta t}\right]$ is bounded in $L^{2}\left(0, T, L^{2}\left(\Omega, L^{2}\left(\mathbb{R}^{d}\right)\right)\right)$ and that $\widetilde{u}^{\Delta t}-u^{\Delta t}$ converges to 0 in $L^{2}\left(0, T, L^{2}\left(\Omega, H^{1}\left(\mathbb{R}^{d}\right)\right)\right)$.

Denote by $u$ a limit point of $u^{\Delta t}$ and $\widetilde{u}^{\Delta t}$ for the weak-* convergence in $L^{\infty}\left(0, T, L^{2}\left(\Omega, H^{1}\left(\mathbb{R}^{d}\right)\right)\right), g_{u}$, respectively $f_{u}$, a limit point of $g\left(u^{\Delta t}\right)$, respectively $f\left(u^{\Delta t}\right)$, for the weak convergence in $L^{2}\left(0, T, L^{2}\left(\Omega, H^{1}\left(\mathbb{R}^{d}\right)\right)\right)$. Since $\widetilde{u}^{\Delta t}-\widetilde{B}^{\Delta t}$ converges weakly in $L^{2}(\Omega, W(0, T))$ where $W(0, T)$ denotes the set of

$L^{2}\left(0, T, H^{1}\left(\mathbb{R}^{d}\right)\right)$-functions $\Psi$ such that $\partial_{t} \Psi \in L^{2}\left(0, T, H^{-1}\left(\mathbb{R}^{d}\right)\right)$ with the common identification of $L^{2}\left(\mathbb{R}^{d}\right)$ with its dual space, $\widetilde{u}^{\Delta t}-\widetilde{B}^{\Delta t}$ converges weakly in $L^{2}\left(\Omega, C\left([0, T], L^{2}\left(\mathbb{R}^{d}\right)\right)\right)$. Thus, for any $t \in[0, T]$, $\left(\widetilde{u}^{\Delta t}-\widetilde{B}^{\Delta t}\right)(t)$ converges weakly in $L^{2}\left(\Omega, L^{2}\left(\mathbb{R}^{d}\right)\right)$.

Note that for $t \in[n \Delta t,(n+1) \Delta t[$, one has

$$
\widetilde{B}^{\Delta t}(t)-\int_{0}^{t} g\left(u^{\Delta t}(s-\Delta t) d W(s)=\left(W^{n+1}-W^{n}\right) g\left(u^{n}\right) \frac{t-n \Delta t}{\Delta t}-\left(W(t)-W^{n}\right) g\left(u^{n}\right) .\right.
$$

Then, thanks to the a priori estimates and the properties of the Brownian motion :

$$
\begin{aligned}
& E\left[\left\|\left(W^{n+1}-W^{n}\right) g\left(u^{n}\right) \frac{t-n \Delta t}{\Delta t}-\left(W(t)-W^{n}\right) g\left(u^{n}\right)\right\|^{2}\right] \\
= & E\left[\left\|g\left(u^{n}\right)\right\|^{2}\right]\left[\frac{(t-n \Delta t)^{2}}{\Delta t}-2 \frac{t-n \Delta t}{\Delta t}(t-n \Delta t)+(t-n \Delta t)\right] \leqslant C \Delta t .
\end{aligned}
$$

${ }^{*}$ We consider that $u^{\Delta t}(s)=u_{0}$ if $s<0$. 
Since $g\left(u^{\Delta t}(.-\Delta t)\right)$, as $g\left(u^{\Delta t}\right)$ converges weakly to some function $g_{u}$ in $L^{2}\left(0, T, L^{2}\left(\Omega, L^{2}\left(\mathbb{R}^{d}\right)\right)\right)$, thanks to the properties of the Itô integral, $\int_{0} g\left(u^{\Delta t}(s-\Delta t) d W(s)\right.$ converges weakly to $\int_{0} g_{u} d W(s)$ in $\mathcal{C}\left([0, T], L^{2}\left(\Omega, L^{2}\left(\mathbb{R}^{d}\right)\right)\right)$, and $\widetilde{B}^{\Delta t}$ does the same. Thus, the weak convergence of $\widetilde{u}^{\Delta t}-\widetilde{B}^{\Delta t}$ is toward $u-\int_{0} g_{u} d W(s)$ and, for any $t, \widetilde{u}^{\Delta t}(t)$ converges weakly in $L^{2}\left(\Omega, L^{2}\left(\mathbb{R}^{d}\right)\right)$ to $u(t)$.

Moreover, for any $v \in H^{1}\left(\mathbb{R}^{d}\right)$, by denoting $\vec{v}^{\Delta t}()=.\sum_{k=1}^{N} \vec{v}(., k \Delta t) \mathbb{1}_{[(k-1) \Delta t, k \Delta t)}($.

$$
\int_{\mathbb{R}^{d}} \partial_{t}\left[\widetilde{u}^{\Delta t}-\widetilde{B}^{\Delta t}\right] v d x+\epsilon \int_{\mathbb{R}^{d}} \nabla u^{\Delta t} \nabla v d x-\int_{\mathbb{R}^{d}} \vec{v}^{\Delta t} f\left(u^{\Delta t}\right) \nabla v d x=0
$$

and at the limit one gets

$$
\int_{\mathbb{R}^{d}} \partial_{t}\left[u-\int_{0}^{t} g_{u} d W(s)\right] v d x+\epsilon \int_{\mathbb{R}^{d}} \nabla u \nabla v d x-\int_{\mathbb{R}^{d}} \vec{v} f_{u} \nabla v d x=0 .
$$

Note that the Itô formula applied to the function $\Psi(t, v)=e^{-c t}\|u\|^{2}$ yields, for any positive $c$ and any $t \in[0, T]$ the following energy equality

$$
\begin{aligned}
& e^{-c t} E\left[\|u(t)\|^{2}\right]+2 \epsilon \int_{0}^{t} e^{-c s} E\left[\|\nabla u\|^{2}\right] d s-2 \int_{0}^{t} E\left[\int_{\mathbb{R}^{d}} e^{-c s} \vec{v} f_{u} \nabla u d x\right] d s \\
= & \left\|u_{0}\right\|^{2}-c \int_{0}^{t} e^{-c s} E\left[\|u(s)\|^{2}\right] d s+\int_{0}^{t} e^{-c s} E\left[\left\|g_{u}\right\|^{2}\right] d s .
\end{aligned}
$$

In addition, one has for any positive $c$ and $n>0$, by multiplying by $e^{-c n \Delta t}$, that

$$
\begin{aligned}
& E\left[\int_{\mathbb{R}^{d}}\left(e^{-c n \Delta t}\left|u_{n+1}\right|^{2}-e^{-c(n-1) \Delta t}\left|u_{n}\right|^{2}\right) d x\right]+\Delta t 2 \epsilon e^{-c n \Delta t} E\left[\int_{\mathbb{R}^{d}}\left|\nabla u_{n+1}\right|^{2} d x\right] \\
\leqslant & \Delta t e^{-c n \Delta t} E\left[\int_{\mathbb{R}^{d}} g^{2}\left(u_{n}\right) d x\right]+\left(e^{-c n \Delta t}-e^{-c(n-1) \Delta t}\right) E\left[\int_{\mathbb{R}^{d}}\left|u_{n}\right|^{2} d x\right] .
\end{aligned}
$$

Adding from 0 to $k$, we get

$$
\begin{aligned}
& e^{-c k \Delta t} E\left[\left\|u_{k+1}\right\|^{2}\right]+\Delta t 2 \epsilon \sum_{n=0}^{k} e^{-c n \Delta t} E\left[\left\|\nabla u_{n+1}\right\|^{2}\right] \\
\leqslant & \left\|u_{0}\right\|^{2}+\Delta t \sum_{n=0}^{k} e^{-c n \Delta t} E\left[\left\|g\left(u_{n}\right)\right\|^{2}\right]-c \Delta t \sum_{n=1}^{k} e^{-c(n+1) \Delta t} E\left[\left\|u_{n}\right\|^{2}\right] .
\end{aligned}
$$

Moreover, by noting that

$$
\begin{aligned}
\int_{0}^{(k+1) \Delta t} e^{-c s} E\left[\left\|\nabla u^{\Delta t}\right\|^{2}\right] d s & \leqslant \sum_{n=0}^{k} e^{-c n \Delta t} E\left[\left\|\nabla u_{n+1}\right\|^{2}\right] \\
\text { that }-c e^{-c \Delta t} \int_{0}^{k \Delta t} e^{-c s} E\left[\left\|u^{\Delta t}\right\|^{2}\right] d s & =-c e^{-c \Delta t} \sum_{n=0}^{k-1} \int_{n \Delta t}^{(n+1) \Delta t} e^{-c s} E\left[\left\|u_{n+1}\right\|^{2}\right] d s \\
& \leqslant-c e^{-c \Delta t} \sum_{n=0}^{k-1} E\left[\left\|u_{n+1}\right\|^{2}\right] \Delta t e^{-c(n+1) \Delta t} \\
& =-c \sum_{n=1}^{k} E\left[\left\|u_{n}\right\|^{2}\right] \Delta t e^{-c(n+1) \Delta t}, \\
\text { and that } \int_{0}^{k \Delta t} e^{-c s} E\left[\left\|g\left(u^{\Delta t}\right)\right\|^{2}\right] d s & =\sum_{n=0}^{k-1} \int_{n \Delta t}^{(n+1) \Delta t} e^{-c s} E\left[\left\|g\left(u_{n+1}\right)\right\|^{2}\right] d s \\
& \geqslant \sum_{n=0}^{k-1} E\left[\left\|g\left(u_{n+1}\right)\right\|^{2}\right] \Delta t e^{-c(n+1) \Delta t} \\
& =-\Delta t\left\|g\left(u_{0}\right)\right\|^{2}+\Delta t \sum_{n=0}^{k} E\left[\left\|g\left(u_{n}\right)\right\|^{2}\right] e^{-c n \Delta t},
\end{aligned}
$$

we deduce from 54

$$
\begin{aligned}
& e^{-c k \Delta t} E\left[\left\|u_{k+1}\right\|^{2}\right]+2 \epsilon \int_{0}^{(k+1) \Delta t} e^{-c s} E\left[\left\|\nabla u^{\Delta t}\right\|^{2}\right] d s \\
\leqslant & \left\|u_{0}\right\|^{2}+\Delta t\left\|g\left(u_{0}\right)\right\|^{2}+\int_{0}^{k \Delta t} e^{-c s} E\left[\left\|g\left(u^{\Delta t}\right)\right\|^{2}\right] d s-c e^{-c \Delta t} \int_{0}^{k \Delta t} e^{-c s} E\left[\left\|u^{\Delta t}\right\|^{2}\right] d s .
\end{aligned}
$$

Now, for $t \in[k \Delta t,(k+1) \Delta t[$, we obtain

$$
\begin{aligned}
& e^{-c t} E\left[\left\|u^{\Delta t}(t)\right\|^{2}\right]+2 \epsilon \int_{0}^{t} e^{-c s} E\left[\left\|\nabla u^{\Delta t}\right\|^{2}\right] d s \\
\leqslant & \left\|u_{0}\right\|^{2}+\Delta t\left\|g\left(u_{0}\right)\right\|^{2}+\int_{0}^{t} e^{-c s} E\left[\left\|g\left(u^{\Delta t}\right)\right\|^{2}\right] d s-c e^{-c \Delta t} \int_{0}^{(t-\Delta t)^{+}} e^{-c s} E\left[\left\|u^{\Delta t}\right\|^{2}\right] d s,
\end{aligned}
$$


and, since $u^{\Delta t}$ is bounded in $L^{\infty}\left(0, T, L^{2}\left(\Omega, L^{2}\left(\mathbb{R}^{d}\right)\right)\right)$, one gets by noting that $\int_{(t-\Delta t)^{+}}^{t} e^{-c s} E\left[\left\|u^{\Delta t}\right\|^{2}\right] d s \leqslant$ $\Delta t\left\|u^{\Delta t}\right\|_{L^{\infty}\left(0, T, L^{2}\left(\Omega \times \mathbb{R}^{d}\right)\right)}^{2}$

$$
\begin{aligned}
& e^{-c t} E\left\|u^{\Delta t}(t)\right\|^{2}+2 \epsilon \int_{0}^{t} e^{-c s} E\left\|\nabla u^{\Delta t}\right\|^{2} d s \\
\leqslant & \left\|u_{0}\right\|^{2}+C \Delta t+\int_{0}^{t} e^{-c s} E\left[\left\|g\left(u^{\Delta t}\right)\right\|^{2}\right] d s-c e^{-c \Delta t} \int_{0}^{t} e^{-c s} E\left[\left\|u^{\Delta t}\right\|^{2}\right] d s .
\end{aligned}
$$

Using this last inequality and the fact that for any $v$ in $H^{1}\left(\mathbb{R}^{d}\right)$ and any $s$ in $[0, T], \int_{\mathbb{R}^{d}} \vec{v}(x, s) f(v) \nabla v d x=0$, one has

$$
\begin{aligned}
& e^{-c t} E\left[\left\|u^{\Delta t}(t)\right\|^{2}\right]+2 \epsilon \int_{0}^{t} e^{-c s} E\left[\left\|\nabla\left(u^{\Delta t}-u\right)\right\|^{2}\right] d s \\
& -2 \int_{0}^{t} e^{-c s} E\left[\int_{\mathbb{R}^{d}} \vec{v}(x, s)\left[f\left(u^{\Delta t}\right)-f(u)\right] \nabla\left(u^{\Delta t}-u\right) d x\right] d s \\
& +4 \epsilon \int_{0}^{t} e^{-c s} E\left[\int_{\mathbb{R}^{d}} \nabla u^{\Delta t} \nabla u d x\right] d s-2 \epsilon \int_{0}^{t} e^{-c s} E\left[\|\nabla u\|^{2}\right] d s \\
\leqslant & \left\|u_{0}\right\|^{2}+C \Delta t+2 \int_{0}^{t} e^{-c s} E\left[\int_{\mathbb{R}^{d}} \vec{v}(x, s) f\left(u^{\Delta t}\right) \nabla u d x\right] d s \\
& +2 \int_{0}^{t} e^{-c s} E\left[\int_{\mathbb{R}^{d}} \vec{v}(x, s) f(u) \nabla u^{\Delta t} d x\right] d s \\
& +2 \int_{0}^{t} e^{-c s} E\left[\int_{\mathbb{R}^{d}} g\left(u^{\Delta t}\right) g(u) d x\right] d s-\int_{0}^{t} e^{-c s} E\left[\|g(u)\|^{2}\right] d s \\
& +\int_{0}^{t} e^{-c s} E\left[\left\|g\left(u^{\Delta t}\right)-g(u)\right\|^{2}\right] d s-c e^{-c \Delta t} \int_{0}^{t} e^{-c s} E\left[\left\|u^{\Delta t}-u\right\|^{2}\right] d s \\
& -2 c e^{-c \Delta t} \int_{0}^{t} e^{-c s} E\left[\int_{\mathbb{R}^{d}} u^{\Delta t} u d x\right] d s+c e^{-c \Delta t} \int_{0}^{t} e^{-c s} E\left[\|u\|^{2}\right] d s .
\end{aligned}
$$

Note that there exists $c=C(V, f, g, \epsilon)>0$ such that, for $\Delta t$ small, one has that

$$
\begin{aligned}
& -2 \epsilon \int_{0}^{t} e^{-c s} E\left[\left\|\nabla\left(u^{\Delta t}-u\right)\right\|^{2}\right] d s+2 \int_{0}^{t} e^{-c s} E\left[\int_{\mathbb{R}^{d}}\left[\vec{v}(x, s)\left\{f\left(u^{\Delta t}\right)-f(u)\right\} \nabla\left(u^{\Delta t}-u\right) d x\right] d s\right. \\
& +\int_{0}^{t} e^{-c s} E\left[\left\|g\left(u^{\Delta t}\right)-g(u)\right\|^{2}\right] d s-c e^{-c \Delta t} \int_{0}^{t} e^{-s} E\left[\left\|u^{\Delta t}-u\right\|^{2}\right] d s \\
\leqslant & -\epsilon \int_{0}^{t} e^{-c s} E\left[\left\|\nabla\left(u^{\Delta t}-u\right)\right\|^{2}\right] d s+\frac{1}{\epsilon} \int_{0}^{t} e^{-c s} E\left[\left\|\vec{v}(x, s)\left(f\left(u^{\Delta t}\right)-f(u)\right)\right\|^{2}\right] d s \\
& +\int_{0}^{t} e^{-c s} E\left[\left\|g\left(u^{\Delta t}\right)-g(u)\right\|^{2}\right] d s-c e^{-c \Delta t} \int_{0}^{t} e^{-c s} E\left[\left\|u^{\Delta t}-u\right\|^{2}\right] d s \\
\leqslant & -\epsilon \int_{0}^{t} e^{-c s} E\left[\left\|\nabla\left(u^{\Delta t}-u\right)\right\|^{2} d s .\right.
\end{aligned}
$$

Indeed, for $c>0$ satisfying $\frac{1}{\epsilon}\left(C_{f} V\right)^{2}+C_{g}^{2} \leqslant c e^{-c \Delta t}$ with $\Delta t>0$ small, one shows that

$\frac{1}{\epsilon} \int_{0}^{t} e^{-c s} E\left[\left\|\vec{v}(x, s)\left(f\left(u^{\Delta t}\right)-f(u)\right)\right\|^{2}\right] d s+\int_{0}^{t} e^{-c s} E\left[\left\|g\left(u^{\Delta t}\right)-g(u)\right\|^{2}\right] d s-c e^{-c \Delta t} \int_{0}^{t} e^{-c s} E\left[\left\|u^{\Delta t}-u\right\|^{2}\right] d s \leqslant 0$.

Thus, for such a choice of $c$ and by integrating 55 with respect to t from 0 to $\mathrm{T}$ one gets:

$$
\begin{aligned}
& \int_{0}^{T} e^{-c t} E\left[\left\|u^{\Delta t}(t)\right\|^{2}\right] t+\epsilon \int_{0}^{T} \int_{0}^{t} e^{-c s} E\left[\left\|\nabla\left(u^{\Delta t}-u\right)\right\|^{2}\right] d s d t \\
\leqslant & T\left\|u_{0}\right\|^{2}+C \Delta t \\
& +2 \int_{0}^{T} \int_{0}^{t} e^{-c s} E\left[\int_{\mathbb{R}^{d}} \vec{v}(x, s) f\left(u^{\Delta t}\right) \nabla u d x\right] d s d t+2 \int_{0}^{T} \int_{0}^{t} e^{-c s} E\left[\int_{\mathbb{R}^{d}} \vec{v}(x, s) f(u) \nabla u^{\Delta t} d x\right] d s d t \\
& +2 \int_{0}^{T} \int_{0}^{t} e^{-c s} E\left[\int_{\mathbb{R}^{d}} g\left(u^{\Delta t}\right) g(u) d x\right] d s d t-\int_{0}^{T} \int_{0}^{t} e^{-c s} E\left[\|g(u)\|^{2}\right] d s d t \\
& -2 c e^{-c \Delta t} \int_{0}^{T} \int_{0}^{t} e^{-c s} E\left[\int_{\mathbb{R}^{d}} u^{\Delta t} u d x\right] d s d t+c e^{-c \Delta t} \int_{0}^{T} \int_{0}^{t} e^{-c s} E\left[\|u\|^{2}\right] d s d t \\
& -4 \epsilon \int_{0}^{T} \int_{0}^{t} e^{-c s} E\left[\int_{\mathbb{R}^{d}} \nabla u^{\Delta t} \nabla u d x\right] d s d t+2 \epsilon \int_{0}^{T} \int_{0}^{t} e^{-c s} E\left[\|\nabla u\|^{2}\right] d s d t .
\end{aligned}
$$

This yields

$$
\begin{aligned}
& \limsup \int_{0}^{T} e^{-c t} E\left[\left\|u^{\Delta t}(t)\right\|^{2}\right] d t \\
\leqslant & \int_{0}^{T}\left\{\left\|u_{0}\right\|^{2}+2 \int_{0}^{t} e^{-c s} E\left[\int_{\mathbb{R}^{d}} \vec{v}(x, s) f_{u} \nabla u d x\right] d s-2 \epsilon \int_{0}^{t} e^{-c s} E\left[\|\nabla u(s)\|^{2}\right] d s-c \int_{0}^{t} e^{-c s} E\left[\|u(s)\|^{2}\right] d s\right\} d t \\
& +2 \int_{0}^{T} \int_{0}^{t} e^{-c s} E\left[\int_{\mathbb{R}^{d}} g_{u} g(u) d x\right] d s d t-\int_{0}^{T} \int_{0}^{t} e^{-c s} E\left[\|g(u)\|^{2}\right] d s d t,
\end{aligned}
$$


and, thanks to the energy equality (53),

$$
\underset{\Delta t}{\limsup } \int_{0}^{T} e^{-c t} E\left[\left\|u^{\Delta t}(t)\right\|^{2}\right] d t+\int_{0}^{T} \int_{0}^{t} e^{-c s} E\left[\left\|g_{u}-g(u)\right\|^{2}\right] d s d t \leqslant \int_{0}^{T} e^{-c t} E\left[\|u(t)\|^{2}\right] d t .
$$

Thus, one gets that $g_{u}=g(u), u^{\Delta t}$ converges to $u$ in $L^{2}\left((0, T) \times \Omega \times \mathbb{R}^{d}\right)$ and $f_{u}=f(u)$. This means that $u$ is a solution and since it depends on $\epsilon>0$ we will denote it $u_{\epsilon}$. Remark that it is a direct proof to show that it is unique.

Then, the stochastic energy asserts that (see for example Grecksch-Tudor GT95] Th. 3.4 p.42):

$$
\begin{gathered}
\left\|u_{\epsilon}(t)\right\|_{L^{2}\left(\mathbb{R}^{d}\right)}^{2}+2 \int_{0}^{t} \int_{\mathbb{R}^{d}}\left[\epsilon\left|\nabla u_{\epsilon}\right|^{2}-\vec{v}(x, s) f\left(u_{\epsilon}\right) \cdot \nabla u_{\epsilon}\right] d x d s \\
=\left\|u_{\epsilon}(0)\right\|_{L^{2}\left(\mathbb{R}^{d}\right)}^{2}+2 \int_{0}^{t} \int_{\mathbb{R}^{d}} u_{\epsilon} g\left(u_{\epsilon}\right) d x d W(s)+\int_{0}^{t} \int_{\mathbb{R}^{d}} g^{2}\left(u_{\epsilon}\right) d x d s .
\end{gathered}
$$

Since $\int_{0}^{t} \int_{\mathbb{R}^{d}} \vec{v}(x, s) f\left(u_{\epsilon}\right) \cdot \nabla u_{\epsilon} d x d s=0$, taking the expectation and using the lemma of Gronwall, there exists $C>0$ such that for all $\epsilon>0$

$$
\left\|u_{\epsilon}\right\|_{L^{\infty}\left(0, T ; L^{2}\left(\Omega \times \mathbb{R}^{d}\right)\right)}^{2}+\epsilon\left\|u_{\epsilon}\right\|_{L^{2}\left((0, T) \times \Omega ; H_{0}^{1}\left(\mathbb{R}^{d}\right)\right)}^{2} \leqslant C .
$$

Finally, as by the existence proof $\partial_{t}\left(u_{\epsilon}-\int_{0}^{t} g\left(u_{\epsilon}\right) d W\right) \in L^{2}(\Omega \times Q)$, we get that $\Delta u_{\epsilon} \in L^{2}(\Omega \times Q)$ and the proof of the proposition is complete.

Proposition 8 If the initial condition $u_{0}^{\epsilon} \in L^{2 p}\left(\mathbb{R}^{d}\right), p \geqslant 1$, then $u_{\epsilon} \in L^{\infty}\left(0, T, L^{2 p}\left(\Omega \times \mathbb{R}^{d}\right)\right)$ as well.

Proof. The proof of this result will not be developed here as it is a straightforward adaptation of the one given in BVW12 Proposition A.5 p.702 to the case of a time-space dependent flux-function.

\section{A.2 Existence and uniqueness of the stochastic entropy solution}

\section{A.2.1 Existence result}

The aim of this section is to show the existence of a measure-valued entropy solution in the sense of Definition 2. To do this, we first consider the viscous parabolic case: assume that for any positive $\epsilon, u_{\epsilon}$ is the solution of the stochastic nonlinear parabolic problem

$$
\left\{\begin{array}{rlrl}
d u_{\epsilon}-\epsilon \Delta u_{\epsilon} d t+\operatorname{div}\left[\vec{v}(x, t) f\left(u_{\epsilon}\right)\right] d t & =g\left(u_{\epsilon}\right) d W & & \text { in } \Omega \times \mathbb{R}^{d} \times(0, T), \\
u_{\epsilon}(\omega, x, 0) & =u_{0}^{\epsilon}(x), & \omega \in \Omega, x \in \mathbb{R}^{d}
\end{array}\right.
$$

where $u_{0}^{\epsilon} \in \mathcal{D}\left(\mathbb{R}^{d}\right)$. Consider $\varphi$ in $\mathcal{D}^{+}\left(\mathbb{R}^{d} \times[0, T)\right)$ and $\eta \in \mathcal{A}$. Using the same technics as in BVW12] (such as Itô formula, chain-rule for Sobolev functions, integration by parts formula, the convexity of $\eta$ and the positivity of $\varphi$ ), we get, $\mathrm{P}$-a.s in $\Omega$

$$
\begin{aligned}
0 \leqslant & \int_{\mathbb{R}^{d}} \eta\left(u_{0}^{\epsilon}\right) \varphi(x, 0) d x+\int_{Q} \eta\left(u_{\epsilon}\right) \partial_{t} \varphi(x, t) d x d t-\epsilon \int_{Q} \eta^{\prime}\left(u_{\epsilon}\right) \nabla u_{\epsilon} \nabla_{x} \varphi(x, t) d x d t \\
& +\int_{Q} \Phi\left(u_{\epsilon}\right) \vec{v}(x, t) \nabla_{x} \varphi(x, t) d x d t+\int_{0}^{T} \int_{\mathbb{R}^{d}} \eta^{\prime}\left(u_{\epsilon}\right) g\left(u_{\epsilon}\right) \varphi(x, t) d x d W(t) \\
& +\frac{1}{2} \int_{Q} g^{2}\left(u_{\epsilon}\right) \eta^{\prime \prime}\left(u_{\epsilon}\right) \varphi(x, t) d x d t
\end{aligned}
$$

where $\Phi$ denotes the entropy flux defined for any $a \in \mathbb{R}$ by $\Phi(a)=\int_{0}^{a} \eta^{\prime}(\sigma) f^{\prime}(\sigma) d \sigma$.

Now we aim to pass to the limit in this inequality when $\epsilon \rightarrow 0$. As for the convergence of the finite volume scheme, the technique is based on the notion of narrow convergence of Young measures. Since $u_{\epsilon}$ is a bounded sequence in $\mathcal{N}_{w}^{2}\left(0, T, L^{2}\left(\mathbb{R}^{d}\right)\right)$, the associated Young measure sequence converges (up to a subsequence still indexed in the same way) to an "entropy process" denoted by $\mathbf{u} \in L^{\infty}\left(0, T, L^{2}\left(\Omega \times \mathbb{R}^{d} \times\right] 0,1[)\right)$. Using the same kind of arguments as in the work of [BVW12], one gets at the limit, P-a.s in $\Omega$, for any $\eta \in \mathcal{A}$ and for any $\varphi \in \mathcal{D}^{+}\left(\mathbb{R}^{d} \times[0, T)\right)$

$\begin{aligned} 0 \leqslant & \int_{\mathbb{R}^{d}} \eta\left(u_{0}\right) \varphi(x, 0) d x+\int_{Q} \int_{0}^{1} \eta(\mathbf{u}(., \alpha)) \partial_{t} \varphi(x, t) d \alpha d x d t+\int_{Q} \int_{0}^{1} \Phi(\mathbf{u}(., \alpha)) \vec{v}(x, t) \nabla_{x} \varphi(x, t) d \alpha d x d t \\ & +\int_{0}^{T} \int_{\mathbb{R}^{d}} \int_{0}^{1} \eta^{\prime}(\mathbf{u}(., \alpha)) g(\mathbf{u}(., \alpha)) \varphi(x, t) d \alpha d x d W(t)+\frac{1}{2} \int_{Q} \int_{0}^{1} g^{2}(\mathbf{u}(., \alpha)) \eta^{\prime \prime}(\mathbf{u}(., \alpha)) \varphi(x, t) d \alpha d x d t .\end{aligned}$

Remark 12 Let us state some properties implicitly satisfied by such an entropy process $\mathbf{u}$. We will not give the details of the proofs of these properties since they are very close to the one developed in [BVW12] and can be adapted straightforward to the case of a time and space dependent flux-function. 
- The entropy process $\mathbf{u}$ is an element of $L^{\infty}\left((0, T), L^{2}\left(\Omega \times \mathbb{R}^{d} \times(0,1)\right)\right)$.

- Moreover, $\mathbf{u}$ satisfies the initial condition in the following sense: for any compact set $K \subset \mathbb{R}^{d}$

$$
\underset{t \rightarrow 0^{+}}{\operatorname{ess} \lim } E\left[\int_{K \times(0,1)}\left|\mathbf{u}(x, t, \alpha)-u_{0}(x)\right| d \alpha d x\right]=0 .
$$

Hence we get the existence of a measure-valued entropy solution in the sense of Definition 2, The aim of the following section is to show the uniqueness of such a solution.

\section{A.2.2 Uniqueness result}

The aim of this subsection is to prove the uniqueness of the measure-valued entropy solution $\mathbf{u}$ of the previous subsection. We will also show that it is the unique entropy solution in the sense of Definition 1 In order to do this, we first show that the following Kato inequality holds :

\section{Proposition 9 (Kato inequality)}

Let $\mathbf{u}$, $\hat{\mathbf{u}}$ be two measure-valued entropy solution to (1) with initial data $u_{0}, \hat{u}_{0} \in L^{2}\left(\mathbb{R}^{d}\right)$ respectively and such that $u$ is obtained as a limit of the sequence of viscous solutions $\left(u_{\epsilon}\right)_{\epsilon>0}$ of Problem (57). Then, for any nonnegative $H^{1}\left(\mathbb{R}^{d} \times[0, T)\right)$-function $\varphi$ with compact support, it holds

$$
\begin{aligned}
0 \leqslant & E\left[\int_{\mathbb{R}^{d}}\left|\hat{u}_{0}(x)-u_{0}(x)\right| \varphi(x, 0) d x\right]+E\left[\int_{Q} \int_{0}^{1} \int_{0}^{1}|\mathbf{u}(x, t, \beta)-\hat{\mathbf{u}}(x, t, \alpha)| \partial_{t} \varphi(x, t) d \alpha d \beta d x d t\right] \\
+ & E\left[\int_{Q} \int_{0}^{1} \int_{0}^{1} \mathcal{F}(\mathbf{u}(x, t, \beta), \hat{\mathbf{u}}(x, t, \alpha)) \vec{v}(x, t) \nabla_{x} \varphi(x, t) d \alpha d \beta d x d t\right],
\end{aligned}
$$

where $\mathcal{F}(a, b)=\operatorname{sgn}_{0}(a-b)(f(a)-f(b))$.

Remark 13 By exploiting the finite propagation speed property for conservation laws with Lipschitz-continuous flux function and choosing $u_{0}=\hat{u}_{0}$, we will deduce from this Kato inequality that $\mathbf{u}=\hat{\mathbf{u}}$ and thus any measurevalued entropy solution is obtained as the limit of solutions $u_{\epsilon}$ of viscous parabolic approximations to (1). This is stated in the following theorem.

Theorem 4 There exists a unique measure-valued solution in the sense of Definition 2 Moreover, it is the unique entropy solution in the sense of Definition 1 .

Proof. (of Theorem 4)

Following BVW12 and using Proposition 9 one shows that for any $R>0, \mathbf{u}(x, t, \beta)=\hat{\mathbf{u}}(x, t, \alpha)$ for almost any $x \in B(0, R), t \in(0, T), \omega \in \Omega, \alpha, \beta \in(0,1)$. Thus, on the one hand $\mathbf{u}=\hat{\mathbf{u}} ;$ on the other hand $\mathbf{u}(x, t, \alpha)=u(x, t)$ is independent of $\alpha$, hence an entropy solution in the sense of Definition 1 .

Proof. (of Proposition 9)

Let us denote by $\mathbf{u}$ the measure-valued entropy solution from the Subsection A.2.1 (a limit point of $\left(u_{\epsilon}\right)$ ) and $\hat{\mathbf{u}}$ any other admissible measure-valued entropy solution, associated respectively to initial conditions $u_{0}$ and $\hat{u}_{0}$ in $L^{2}\left(\mathbb{R}^{d}\right)$.

Consider $k \in \mathbb{R}, \eta \in \mathcal{A}, \varphi$ in $\mathcal{D}^{+}\left(\mathbb{R}^{d} \times[0, T)\right), K \subset \mathbb{R}^{d}$ a compact set such that $\operatorname{supp} \varphi(., t) \subset K$ and denote by $G(x, y, t, s)=\varphi(y, s) \rho_{m}(x-y) \rho_{n}(t-s)$ where $\rho_{m}$ and $\rho_{n}$ denote the usual mollifier sequences in $\mathbb{R}^{d}$ and $\mathbb{R}$, respectively, with $\operatorname{supp} \rho_{n} \subset\left[-\frac{2}{n}, 0\right]$. Denote also by $\rho_{l}$ a mollifier sequence in $\mathbb{R}$ and for convenience set $p=(x, t, \alpha)$. Finally let us denote by $\mathcal{F}^{\eta}(a, b)=\int_{b}^{a} \eta^{\prime}(\sigma-b) f^{\prime}(\sigma) d \sigma$.

Since $\hat{\mathbf{u}}$ is a measure-valued entropy solution, it satisfies the entropy inequality given by Definition 2 By considering the test function $G$ and the entropy $\eta(.-k)$ in such a formulation, multiplying it by $\rho_{l}\left(u_{\epsilon}(y, s)-k\right)$ and integrating $k$ over $\mathbb{R}$ and with respect to variables $(y, s)$, we get, on the one hand by taking the expectation that

$$
0 \leqslant I_{1}+I_{2}+I_{3}+I_{4}+I_{5}+I_{6}+I_{7},
$$


where

$$
\begin{aligned}
& I_{1}=E\left[\int_{Q} \int_{\mathbb{R}} \int_{\mathbb{R}^{d}} \eta\left(\hat{u}_{0}(x)-k\right) \varphi(y, s) \rho_{n}(-s) \rho_{m}(x-y) d x \rho_{l}\left(u_{\epsilon}(y, s)-k\right) d k d y d s\right] \\
& I_{2}=E\left[\int_{Q} \int_{\mathbb{R}} \int_{0}^{1} \int_{Q} \eta(\hat{\mathbf{u}}(p)-k) \rho_{n}(t-s) \partial_{t} \varphi(y, s) \rho_{m}(x-y) d p \rho_{l}\left(u_{\epsilon}(y, s)-k\right) d k d y d s\right] \\
& I_{3}=E\left[\int_{Q} \int_{\mathbb{R}} \int_{0}^{1} \int_{Q} \eta(\hat{\mathbf{u}}(p)-k) \varphi(y, s) \partial_{t} \rho_{n}(t-s) \rho_{m}(x-y) d p \rho_{l}\left(u_{\epsilon}(y, s)-k\right) d k d y d s\right] \\
& I_{4}=E\left[\int_{Q} \int_{\mathbb{R}} \int_{0}^{1} \int_{Q} \mathcal{F}^{\eta}(\hat{\mathbf{u}}(p), k) \vec{v}(x, t) \rho_{m}(x-y) \nabla_{x} \varphi(y, s) \rho_{n}(t-s) d p \rho_{l}\left(u_{\epsilon}(y, s)-k\right) d k d y d s\right] \\
& I_{5}=E\left[\int_{Q} \int_{\mathbb{R}} \int_{0}^{1} \int_{Q} \mathcal{F}^{\eta}(\hat{\mathbf{u}}(p), k) \vec{v}(x, t) \nabla_{x} \rho_{m}(x-y) \rho_{n}(t-s) \varphi(y, s) d p \rho_{l}\left(u_{\epsilon}(y, s)-k\right) d k d y d s\right] \\
& I_{6}=\frac{1}{2} E\left[\int_{Q} \int_{\mathbb{R}} \int_{0}^{1} \int_{Q} g^{2}(\hat{\mathbf{u}}(p)) \eta^{\prime \prime}(\hat{\mathbf{u}}(p)-k) \rho_{m}(x-y) \rho_{n}(t-s) \varphi(y, s) d p \rho_{l}\left(u_{\epsilon}(y, s)-k\right) d k d y d s\right] \\
& I_{7}=E\left[\int_{Q} \int_{\mathbb{R}} \int_{0}^{T} \int_{\mathbb{R}^{d}} \int_{0}^{1} \eta^{\prime}(\hat{\mathbf{u}}(p)-k) g(\hat{\mathbf{u}}(p)) d \alpha \varphi(y, s) \rho_{m}(x-y) \rho_{n}(t-s) d x d W(t) \rho_{l}\left(u_{\epsilon}(y, s)-k\right) d k d y d s\right] .
\end{aligned}
$$

On the other hand, since $u_{\epsilon}$ is a viscous solution of Problem (48), by considering also the test function $G$ and the entropy $\eta(.-k)$ in the inequality $(58)$ satisfied by $u_{\epsilon}$, multiplying it by $\rho_{l}(\hat{\mathbf{u}}(p)-k)$ and integrating $k$ over $\mathbb{R}, \alpha$ between 0 and 1 and with respect to variables $(x, t)$, taking the expectation, one gets that

$$
0 \leqslant J_{1}+J_{2}+J_{3}+J_{4}+J_{5}+J_{6}+J_{7}+J_{8}+J_{9}
$$

where

$$
\begin{aligned}
& J_{1}=E\left[\int_{0}^{1} \int_{Q} \int_{\mathbb{R}} \int_{\mathbb{R}^{d}} \eta\left(u_{0}^{\epsilon}(y)-k\right) \varphi(0, y) \rho_{n}(t) \rho_{m}(x-y) d y \rho_{l}(\hat{\mathbf{u}}(p)-k) d k d p\right] \\
& J_{2}=E\left[\int_{0}^{1} \int_{Q} \int_{\mathbb{R}} \int_{Q} \eta\left(u_{\epsilon}(y, s)-k\right) \rho_{n}(t-s) \partial_{s} \varphi(y, s) \rho_{m}(x-y) \rho_{l}(\hat{\mathbf{u}}(p)-k) d k d y d s d p\right] \\
& J_{3}=E\left[\int_{0}^{1} \int_{Q} \int_{\mathbb{R}} \int_{Q} \eta\left(u_{\epsilon}(y, s)-k\right) \varphi(y, s) \partial_{s} \rho_{n}(t-s) \rho_{m}(x-y) \rho_{l}(\hat{\mathbf{u}}(p)-k) d k d y d s d p\right] \\
& J_{4}=-\epsilon E\left[\int_{0}^{1} \int_{Q} \int_{\mathbb{R}} \int_{Q} \eta^{\prime}\left(u_{\epsilon}(y, s)-k\right) \rho_{m}(x-y) \nabla_{y} u_{\epsilon}(y, s) \nabla_{y} \varphi(y, s) \rho_{n}(t-s) \rho_{l}(\hat{\mathbf{u}}(p)-k) d k d y d s d p\right] \\
& J_{5}=-\epsilon E\left[\int_{0}^{1} \int_{Q} \int_{\mathbb{R}} \int_{Q} \eta^{\prime}\left(u_{\epsilon}(y, s)-k\right) \varphi(y, s) \nabla_{y} u_{\epsilon}(y, s) \nabla_{y} \rho_{m}(x-y) \rho_{n}(t-s) \rho_{l}(\hat{\mathbf{u}}(p)-k) d k d y d s d p\right] \\
& J_{6}=E\left[\int_{0}^{1} \int_{Q} \int_{\mathbb{R}} \int_{Q} \mathcal{F}^{\eta}\left(u_{\epsilon}(y, s), k\right) \vec{v}(y, s) \rho_{m}(x-y) \nabla_{y} \varphi(y, s) \rho_{n}(t-s) \rho_{l}(\hat{\mathbf{u}}(p)-k) d k d y d s d p\right] \\
& J_{7}=E\left[\int_{0}^{1} \int_{Q} \int_{\mathbb{R}} \int_{Q} \mathcal{F}^{\eta}\left(u_{\epsilon}(y, s), k\right) \vec{v}(y, s) \varphi(y, s) \nabla_{y} \rho_{m}(x-y) \rho_{n}(t-s) \rho_{l}(\hat{\mathbf{u}}(p)-k) d k d y d s d p\right] \\
& J_{8}=\frac{1}{2} E\left[\int_{0}^{1} \int_{Q} \int_{\mathbb{R}} \int_{Q} g^{2}\left(u_{\epsilon}(y, s)\right) \eta^{\prime \prime}\left(u_{\epsilon}(y, s)-k\right) \rho_{m}(x-y) \rho_{n}(t-s) \varphi(y, s) \rho_{l}(\hat{\mathbf{u}}(p)-k) d k d y d s d p\right] \\
& J_{9}=E\left[\int_{0}^{1} \int_{Q} \int_{\mathbb{R}} \int_{0}^{T} \int_{\mathbb{R}^{d}} \eta^{\prime}\left(u_{\epsilon}(y, s)-k\right) g\left(u_{\epsilon}(y, s)\right) \varphi(y, s) \rho_{m}(x-y) \rho_{n}(t-s) d y d W(s) \rho_{l}(\hat{\mathbf{u}}(p)-k) d k d p\right] .
\end{aligned}
$$

Summing up the preceding two inequalities, our aim is now to pass to the limit in the following order: $n \rightarrow \infty$ (convolution in time), $l \rightarrow \infty, \eta \rightarrow|\cdot|, \epsilon \rightarrow 0$ and finally $m \rightarrow \infty$ (convolution in space). In the following, as a uniform approximation of the absolute value function, we choose $\eta=\eta_{\delta} \in \mathcal{A}$ with $\eta_{\delta}^{\prime}(r)=1$ for $r>\delta, \eta_{\delta}^{\prime}(r)=\sin \left(\frac{\pi}{2 \delta} r\right)$ if $|r| \leqslant \delta$ and $\eta_{\delta}^{\prime}(r)=-1$ for $r<-\delta$.

Note that this convergence study has been proved in details in the work of Bauzet-VALLET-WitTBOLD BVW12 in the case where the vector $\vec{v}$ does not depend on the time and the space variable. Thus, we will only develop here the proof of convergence of terms involving the flux function $\vec{v}(x, t) f($.$) , i.e. I_{4}+J_{6}$ and $I_{5}+J_{7}$.

(1) Since $\operatorname{supp} \rho_{n} \subset\left[-\frac{2}{n}, 0\right]$,

$$
\begin{gathered}
I_{1}+J_{1} \quad=\quad E\left[\int_{Q} \int_{\mathbb{R}} \int_{\mathbb{R}^{d}} \eta\left(\hat{u}_{0}(x)-k\right) \varphi(y, s) \rho_{n}(-s) \rho_{m}(x-y) d x \rho_{l}\left(u_{\epsilon}(y, s)-k\right) d k d y d s\right] \\
\underset{n, l, \eta, \epsilon, m}{\longrightarrow} E\left[\int_{\mathbb{R}^{d}}\left|\hat{u}_{0}(x)-u_{0}(x)\right| \varphi(x, 0) d x\right] .
\end{gathered}
$$

(2) As $\varphi$ is a function of variables $(y, s)$

$$
\begin{gathered}
I_{2}+J_{2} \quad=\quad E\left[\int_{0}^{1} \int_{Q} \int_{\mathbb{R}} \int_{Q} \eta\left(u_{\epsilon}(y, s)-k\right) \rho_{n}(t-s) \partial_{s} \varphi(y, s) \rho_{m}(x-y) \rho_{l}(\hat{\mathbf{u}}(p)-k) d y d s d k d p\right] \\
\underset{n, l, \eta, \epsilon, m}{\longrightarrow} E\left[\int_{Q} \int_{0}^{1} \int_{0}^{1}|\mathbf{u}(y, s, \beta)-\hat{\mathbf{u}}(y, s, \alpha)| \partial_{s} \varphi(y, s) d \alpha d \beta d y d s\right] .
\end{gathered}
$$


(3) Since $\eta$ and $\rho_{l}$ are even functions, by setting $\tau=u_{\epsilon}(y, s)-k$ and $\sigma=-(\hat{\mathbf{u}}(p)-k)$ one shows that

$$
I_{3}+J_{3}=0 \text {. }
$$

(4) Thanks to Proposition $7 \epsilon \nabla u_{\epsilon}$ converges to 0 in $L^{2}\left((0, T) \times \Omega, L^{2}\left(\mathbb{R}^{d}\right)\right)$ when $\epsilon \rightarrow 0$ and so

$$
\begin{aligned}
J_{4}+J_{5}= & -\epsilon E\left[\int_{0}^{1} \int_{Q} \int_{\mathbb{R}} \int_{Q} \eta^{\prime}\left(u_{\epsilon}(y, s)-k\right) \rho_{m}(x-y) \nabla_{y} u_{\epsilon}(y, s) \nabla_{y} \varphi(y, s) \rho_{n}(t-s) d y d s \rho_{l}(\hat{\mathbf{u}}(p)-k) d k d p\right] \\
& -\epsilon E\left[\int_{0}^{1} \int_{Q} \int_{\mathbb{R}} \int_{Q} \eta^{\prime}\left(u_{\epsilon}(y, s)-k\right) \varphi(y, s) \nabla_{y} u_{\epsilon}(y, s) \nabla_{y} \rho_{m}(x-y) \rho_{n}(t-s) d y d s \rho_{l}(\hat{\mathbf{u}}(p)-k) d k d p\right]
\end{aligned}
$$

$$
\underset{n, l, \eta, \epsilon}{\longrightarrow} 0
$$

(5) Since $\varphi$ is a function of variables $(y, s)$

$$
\begin{gathered}
I_{4}+J_{6} \quad=\quad E\left[\int_{0}^{1} \int_{Q} \int_{\mathbb{R}} \int_{Q} \mathcal{F}^{\eta}\left(u_{\epsilon}(y, s), k\right) \vec{v}(y, s) \rho_{m}(x-y) \nabla_{y} \varphi(y, s) \rho_{n}(t-s) d y d s \rho_{l}(\hat{\mathbf{u}}(p)-k) d k d p\right] \\
\underset{n, l, \eta, \epsilon, m}{\longrightarrow} E\left[\int_{Q} \int_{0}^{1} \int_{0}^{1} \mathcal{F}(\mathbf{u}(x, t, \beta), \hat{\mathbf{u}}(x, t, \alpha)) \vec{v}(x, t) \nabla_{x} \varphi(x, t) d \beta d \alpha d x d t\right] .
\end{gathered}
$$

where $\mathcal{F}(a, b):=\operatorname{sgn}_{0}(a-b)(f(a)-f(b))$.

Indeed, let us justify the passages to the limit in detail.

\section{- Limit as $n \rightarrow \infty$ :}

$$
\begin{aligned}
\mathcal{A}_{1}= & E\left[\int_{0}^{1} \int_{Q} \int_{\mathbb{R}} \int_{Q}\left\{\mathcal{F}^{\eta}\left(u_{\epsilon}(y, s), k\right)-\mathcal{F}^{\eta}\left(u_{\epsilon}(y, t), k\right)\right\} \vec{v}(y, s) \nabla_{y} \varphi(y, s) \rho_{m}(x-y) \rho_{n}(t-s) d y d s \rho_{l}(\hat{\mathbf{u}}(p)-k) d k d p\right] \\
+ & E\left[\int_{0}^{1} \int_{Q} \int_{\mathbb{R}} \int_{Q} \mathcal{F}^{\eta}\left(u_{\epsilon}(y, t), k\right) \vec{v}(y, s) \rho_{m}(x-y) \nabla_{y}[\varphi(y, s)-\varphi(y, t)] \rho_{n}(t-s) d y d s \rho_{l}(\hat{\mathbf{u}}(p)-k) d k d p\right] \\
+ & E\left[\int_{0}^{1} \int_{Q} \int_{\mathbb{R}} \int_{Q} \mathcal{F}^{\eta}\left(u_{\epsilon}(y, t), k\right)[\vec{v}(y, s)-\vec{v}(y, t)] \rho_{m}(x-y) \nabla_{y} \varphi(y, t) \rho_{n}(t-s) d y d s \rho_{l}(\hat{\mathbf{u}}(p)-k) d k d p\right] \\
& -E\left[\int_{0}^{1} \int_{Q} \int_{\mathbb{R}} \int_{\mathbb{R}^{d}} \mathcal{F}^{\eta}\left(u_{\epsilon}(y, t), k\right) \vec{v}(y, t) \rho_{m}(x-y) \nabla_{y} \varphi(y, t) \rho_{l}(\hat{\mathbf{u}}(p)-k)\left(1-\int_{0}^{T} \rho_{n}(t-s) d s\right) d y d k d p\right] .
\end{aligned}
$$

Since $\mathcal{F}^{\eta}(\cdot, k)$ is a Lipschitz-continuous function with the same Lipschitz constant as $f$ denoted $C_{f}$, $\varphi \in \mathcal{D}^{+}\left(\mathbb{R}^{d} \times[0, T)\right)$, and $0 \leqslant 1-\int_{0}^{T} \rho_{n}(t-s) d s \leqslant 1_{(T-2 / n, T)}$ a.e. on $(0, T)$ and $\left|\eta^{\prime}(r)\right|=\left|\eta_{\delta}^{\prime}(r)\right| \leqslant 1$ for all $r \in \mathbb{R}^{d}$, we get

$$
\begin{aligned}
\left|\mathcal{A}_{1}\right| \leqslant \quad & c\left(C_{f}, \varphi, V, \vec{v}\right)\left\{E\left[\int_{0}^{T} \int_{K} \int_{0}^{T}\left|u_{\epsilon}(y, s)-u_{\epsilon}(y, t)\right| \rho_{n}(t-s) d s d y d t\right]\right. \\
& +\frac{1}{n} E\left[\int_{0}^{T} \int_{K} \int_{0}^{1}\left[\left|u_{\epsilon}(x, t)\right|+|\hat{\mathbf{u}}(x, t, \alpha)|+\mathbb{1}_{K}(x)\right] d \alpha d x d t\right] \\
& \left.+E\left[\int_{T-2 / n}^{T} \int_{K} \int_{0}^{1}\left[\left|u_{\epsilon}(x, t)\right|+|\hat{\mathbf{u}}(x, t, \alpha)|+\mathbb{1}_{K}(x)\right] d \alpha d x d t\right]\right\} \\
\underset{n \rightarrow \infty}{\longrightarrow} & 0 .
\end{aligned}
$$

- Limit as $l \rightarrow \infty$ :

$$
\mathcal{A}_{2}=E\left[\int_{0}^{1} \int_{Q} \int_{\mathbb{R}} \int_{\mathbb{R}^{d}}\left(\mathcal{F}^{\eta}\left(u_{\epsilon}(y, t), k\right)-\mathcal{F}^{\eta}\left(u_{\epsilon}(y, t), \hat{\mathbf{u}}(p)\right)\right) \vec{v}(y, t) \rho_{m}(x-y) \nabla_{y} \varphi(y, t) \rho_{l}(\hat{\mathbf{u}}(p)-k) d y d k d p\right] .
$$

Since $\mathcal{F}^{\eta}$ is Lipschitz-continuous in its second variable, uniformly with respect to the first variable, we can estimate

$$
\begin{aligned}
\left|\mathcal{A}_{2}\right| & \leqslant \frac{V c\left(\mathcal{F}^{\eta}\right)}{l} \int_{Q}\left|\nabla_{y} \varphi(y, t)\right| d y d t \\
\underset{l \rightarrow \infty}{\longrightarrow} 0 . & .
\end{aligned}
$$

- Limit as $\eta=\eta_{\delta} \rightarrow|\cdot|$ :

As for $\eta=\eta_{\delta}$, we have $\left|\mathcal{F}^{\eta}(r, s)-\mathcal{F}(r, s)\right| \leqslant \delta C_{f}$ for any $r, s \in \mathbb{R}$, we can easily estimate

$$
\begin{aligned}
\mathcal{A}_{3} & :=E\left[\int_{0}^{1} \int_{\mathbb{R}^{d}} \int_{Q}\left(\mathcal{F}^{\eta}\left(u_{\epsilon}(y, t), \hat{\mathbf{u}}(p)\right)-\mathcal{F}\left(u_{\epsilon}(y, t), \hat{\mathbf{u}}(p)\right)\right) \vec{v}(y, t) \rho_{m}(x-y) \nabla_{y} \varphi(y, t) d y d p\right] \\
\text { by } & \\
\left|\mathcal{A}_{3}\right| & \leqslant \delta C_{f} V \int_{Q} \int_{\mathbb{R}^{d}}\left|\nabla_{y} \varphi(y, t)\right| \rho_{m}(x-y) d y d x d t \leqslant \delta C_{f} V \int_{Q}\left|\nabla_{y} \varphi(y, t)\right| d y d t
\end{aligned}
$$

which goes to 0 as $\delta \rightarrow 0$. 
- Limit as $\epsilon \rightarrow 0$ :

By denoting $\mathcal{G}(k, y, t)=\int_{\mathbb{R}^{d}} \int_{0}^{1} \mathcal{F}(k, \hat{\mathbf{u}}(x, t, \alpha)) \vec{v}(y, t) \rho_{m}(x-y) \nabla_{y} \varphi(y, t) d \alpha d x$, which is a Carathéodory function with $\operatorname{supp} \mathcal{G}(k, \cdot, t) \subset K$, one gets at the limit

$$
\begin{aligned}
& E\left[\int_{0}^{1} \int_{Q} \int_{\mathbb{R}^{d}} \mathcal{F}\left(u_{\epsilon}(y, t), \hat{\mathbf{u}}(x, t, \alpha)\right) \vec{v}(y, t) \rho_{m}(x-y) \nabla_{y} \varphi(y, t) d y d p\right] \\
=\quad & E\left[\int_{Q} \mathcal{G}\left(u_{\epsilon}(y, t), y, t\right) d y d t\right] \\
\underset{\epsilon \rightarrow 0}{\longrightarrow} & E\left[\int_{0}^{1} \int_{Q} \int_{\mathbb{R}^{d}} \int_{0}^{1} \mathcal{F}(\mathbf{u}(y, t, \beta), \hat{\mathbf{u}}(x, t, \alpha)) \vec{v}(y, t) \rho_{m}(x-y) \nabla_{y} \varphi(y, t) d \beta d y d p\right] .
\end{aligned}
$$

- Limit as $m \rightarrow \infty$ :

$$
\begin{aligned}
\mathcal{A}_{5} & =E\left[\int_{0}^{1} \int_{Q} \int_{0}^{1} \int_{\mathbb{R}^{d}}(\mathcal{F}(\mathbf{u}(y, t, \beta), \hat{\mathbf{u}}(x, t, \alpha))-\mathcal{F}(\mathbf{u}(x, t, \beta), \hat{\mathbf{u}}(x, t, \alpha))) \vec{v}(y, t) \rho_{m}(x-y) \nabla_{y} \varphi(y, t) d y d \beta d p\right] . \\
\left|\mathcal{A}_{5}\right| & \leqslant \operatorname{Vc}(\mathcal{F}, \varphi) E\left[\int_{Q} \int_{0}^{1} \int_{K}|\mathbf{u}(y, t, \beta)-\mathbf{u}(x, t, \beta)| \rho_{m}(x-y) d y d \beta d x d t\right] \\
\underset{m \rightarrow \infty}{\longrightarrow} & 0 .
\end{aligned}
$$

(6) Now let us consider $I_{5}+J_{7}$ : as $\nabla_{y} \rho_{m}(x-y)=-\nabla_{x} \rho_{m}(x-y)$ we get

$$
\begin{aligned}
& \left|I_{5}+J_{7}\right| \\
\leqslant & \left|E\left[\int_{Q} \int_{\mathbb{R}} \int_{0}^{1} \int_{Q}\left\{\mathcal{F}^{\eta}\left(\hat{\mathbf{u}}(p), u_{\epsilon}(y, s)-k\right)-\mathcal{F}^{\eta}\left(u_{\epsilon}(y, s), \hat{\mathbf{u}}(p)-k\right)\right\} \vec{v}(x, t) \varphi(y, s) \nabla_{x} \rho_{m}(x-y) \rho_{n}(t-s) d p \rho_{l}(k) d k d y d s\right]\right| \\
& +\left|E\left[\int_{Q} \int_{\mathbb{R}} \int_{0}^{1} \int_{Q} \mathcal{F}^{\eta}\left(u_{\epsilon}(y, s), \hat{\mathbf{u}}(p)-k\right)[\vec{v}(x, t)-\vec{v}(y, s)] \varphi(y, s) \nabla_{x} \rho_{m}(x-y) \rho_{n}(t-s) d p \rho_{l}(k) d k d y d s\right]\right| .
\end{aligned}
$$

Note that since $\operatorname{div}[\vec{v}(x, t)]=0 \forall(x, t) \in \mathbb{R}^{d} \times[0, T]$, an integration by part with respect to $x$ allows us to show that

$E\left[\int_{Q} \int_{\mathbb{R}} \int_{0}^{1} \int_{Q} \mathcal{F}^{\eta}\left(u_{\epsilon}(y, s), \hat{\mathbf{u}}(y, s, \alpha)-k\right)[\vec{v}(x, t)-\vec{v}(y, s)] \varphi(y, s) \nabla_{x} \rho_{m}(x-y) \rho_{n}(t-s) d p \rho_{l}(k) d k d y d s\right]=0$.

In this way,

$$
\begin{gathered}
\left|I_{5}+J_{7}\right| \\
\leqslant\left|E\left[\int_{Q} \int_{\mathbb{R}} \int_{0}^{1} \int_{Q}\left\{\mathcal{F}^{\eta}\left(\hat{\mathbf{u}}(p), u_{\epsilon}(y, s)-k\right)-\mathcal{F}^{\eta}\left(u_{\epsilon}(y, s), \hat{\mathbf{u}}(p)-k\right)\right\} \vec{v}(x, t) \varphi(y, s) \nabla_{x} \rho_{m}(x-y) \rho_{n}(t-s) d p \rho_{l}(k) d k d y d s\right]\right| \\
+\mid E\left[\int _ { \mathbb { R } } \int _ { 0 } ^ { 1 } \int _ { Q ^ { 2 } } \left\{\mathcal{F}^{\eta}\left(u_{\epsilon}(y, s), \hat{\mathbf{u}}(p)-k\right)-\right.\right. \\
\left.\mathcal{F}^{\eta}\left(u_{\epsilon}(y, s), \hat{\mathbf{u}}(y, s, \alpha)-k\right)\right\}[\vec{v}(x, t)-\vec{v}(y, s)] \\
\left.\times \varphi(y, s) \nabla_{x} \rho_{m}(x-y) \rho_{n}(t-s) d y d s d p \rho_{l}(k) d k\right] \mid .
\end{gathered}
$$

Using the symmetry of $\mathcal{F}($ i.e. $\mathcal{F}(r, s)=\mathcal{F}(s, r))$, the fact that for $\eta=\eta_{\delta}:\left|\mathcal{F}^{\eta}(r, s)-\mathcal{F}(r, s)\right| \leqslant \delta C_{f}$, the Lipschitz-continuity of $\mathcal{F}$ with respect to both of its variables and the estimate $\left|\nabla_{x} \rho_{m}(x)\right| \leqslant c m^{d+1}$, we get

$$
\begin{aligned}
& \left|I_{5}+J_{7}\right| \\
\leqslant & E\left[\int_{Q} \int_{\mathbb{R}} \int_{0}^{1} \int_{Q}\left|\mathcal{F}^{\eta}\left(\hat{\mathbf{u}}(p), u_{\epsilon}(y, s)-k\right)-\mathcal{F}\left(\hat{\mathbf{u}}(p), u_{\epsilon}(y, s)-k\right)\right|\left|\vec{v}(x, t) \nabla_{x} \rho_{m}(x-y)\right| \rho_{n}(t-s) \varphi(y, s) d p \rho_{l}(k) d k d y d s\right] \\
+ & E\left[\int_{Q} \int_{\mathbb{R}} \int_{0}^{1} \int_{Q}\left|\mathcal{F}\left(\hat{\mathbf{u}}(p), u_{\epsilon}(y, s)-k\right)-\mathcal{F}\left(u_{\epsilon}(y, s), \hat{\mathbf{u}}(p)-k\right) \| \vec{v}(x, t) \nabla_{x} \rho_{m}(x-y)\right| \rho_{n}(t-s) \varphi(y, s) d p \rho_{l}(k) d k d y d s\right] \\
& +E\left[\int_{Q} \int_{\mathbb{R}} \int_{0}^{1} \int_{Q}\left|\mathcal{F}\left(u_{\epsilon}(y, s), \hat{\mathbf{u}}(p)-k\right)-\mathcal{F}^{\eta}\left(u_{\epsilon}(y, s), \hat{\mathbf{u}}(p)-k\right) \| \vec{v}(x, t) \nabla_{x} \rho_{m}(x-y)\right| \rho_{n}(t-s) \varphi(y, s) d p \rho_{l}(k) d k d y d s\right] \\
& +C_{f} E\left[\int_{Q} \int_{\mathbb{R}} \int_{0}^{1} \int_{Q}|\hat{\mathbf{u}}(x, t, \alpha)-\hat{\mathbf{u}}(y, s, \alpha)|\left|[\vec{v}(x, t)-\vec{v}(y, s)] \nabla_{x} \rho_{m}(x-y)\right| \rho_{n}(t-s) \varphi(y, s) d p \rho_{l}(k) d k d y d s\right] \\
\leqslant & 2 c\left(C_{f}, V, \varphi, m\right) \delta+2 c\left(C_{f}, V, \varphi\right) \frac{1}{l} \int_{K} \int_{B(y, 1 / m)}\left|\nabla_{x} \rho_{m}(x-y)\right| d x d y \\
& +2 c\left(C_{f}, \vec{v}, \varphi, m^{d+1}\right) E\left[\int_{0}^{T} \int_{0}^{T} \int_{K} \int_{0}^{1}|\hat{\mathbf{u}}(x, t, \alpha)-\hat{\mathbf{u}}(x, s, \alpha)| \rho_{n}(t-s) d \alpha d x d t d s\right] \\
& +\frac{1}{n} c\left(C_{f}, \vec{v}, \varphi, m^{d+1},\|\hat{\mathbf{u}}\|_{L_{\mathrm{loc}}^{1}(\Omega \times Q \times(0,1))}\right) \\
& +c\left(C_{f}, \vec{v}, \varphi\right) m^{d} E\left[\int_{0}^{T} \int_{K} \int_{B(0,1 / m)} \int_{0}^{1}|\hat{\mathbf{u}}(y+z, s, \alpha)-\hat{\mathbf{u}}(y, s, \alpha)| d \alpha d z d y d s\right]
\end{aligned}
$$


hence $\limsup \left|I_{5}+I_{7}\right|=0$.

(7) As in BVW12 one shows that

$$
\begin{aligned}
I_{6}+J_{8}= & \frac{1}{2} E\left[\int_{Q} \int_{\mathbb{R}} \int_{0}^{1} \int_{Q} g^{2}(\hat{\mathbf{u}}(p)) \eta^{\prime \prime}(\hat{\mathbf{u}}(p)-k) \rho_{m}(x-y) \rho_{n}(t-s) \varphi(y, s) d p \rho_{l}\left(u_{\epsilon}(y, s)-k\right) d k d y d s\right] \\
& +\frac{1}{2} E\left[\int_{0}^{1} \int_{Q} \int_{\mathbb{R}} \int_{Q} g^{2}\left(u_{\epsilon}(y, s)\right) \eta^{\prime \prime}\left(u_{\epsilon}(y, s)-k\right) \rho_{m}(x-y) \rho_{n}(t-s) \varphi(y, s) d y d s \rho_{l}(\hat{\mathbf{u}}(p)-k) d k d p\right] \\
\underset{n, l \rightarrow \infty}{\longrightarrow} & \frac{1}{2} E\left[\int_{\mathbb{R}^{d}} \int_{0}^{1} \int_{Q} g^{2}(\hat{\mathbf{u}}(p)) \eta^{\prime \prime}\left(\hat{\mathbf{u}}(p)-u_{\epsilon}(y, t)\right) \rho_{m}(x-y) \varphi(y, t) d p d y\right] \\
& +\frac{1}{2} E\left[\int_{\mathbb{R}^{d}} \int_{0}^{1} \int_{Q} g^{2}\left(u_{\epsilon}(y, t)\right) \eta^{\prime \prime}\left(u_{\epsilon}(y, t)-\hat{\mathbf{u}}(p)\right) \rho_{m}(x-y) \varphi(y, t) d p d y\right] .
\end{aligned}
$$

Note that it is not possible to pass to the limit with $\eta \rightarrow|\cdot|$ in the preceding terms $\lim _{l} \lim _{n} I_{6}+I_{8}$, as we ignore the limit of $\eta^{\prime \prime}$. Instead and as in BVW12, we keep this term for the moment. We will combine it below with corresponding integrals resulting from the stochastic integrals and show that the sum of these terms vanishes as $\eta \rightarrow|\cdot|$.

(8) We come now to the estimate of the stochastic integrals. Using the same techniques as in BVW12 (p.687), which use properties of the stochastic integral and the Itô formula, one shows that $I_{7}+J_{9}$ can be written in the following way

$$
\begin{aligned}
I_{7}+J_{9}=- & E\left[\int_{Q} \int_{\mathbb{R}} \int_{\mathbb{R}^{d}} \int_{s-2 / n}^{s} \int_{0}^{1} \eta^{\prime \prime}(\hat{\mathbf{u}}(p)-k) g(\hat{\mathbf{u}}(p)) d \alpha \rho_{n}(t-s) d W(t) \varphi(y, s) \rho_{m}(x-y) d x\right. \\
& \times\left(\int_{s-\frac{2}{n}}^{s} \rho_{l}\left(u_{\epsilon}(y, \sigma)-k\right)\left\{\epsilon \Delta u_{\epsilon}(y, \sigma)-\operatorname{div}\left[\vec{v}(y, \sigma) f\left(u_{\epsilon}(y, \sigma)\right)\right]\right\} d \sigma\right. \\
& \left.\left.+\int_{s-\frac{2}{n}}^{s} \rho_{l}\left(u_{\epsilon}(\sigma, y)-k\right) g\left(u_{\epsilon}(\sigma, y)\right) d W(\sigma)+\frac{1}{2} \int_{s-\frac{2}{n}}^{s} \rho_{l}^{\prime}\left(u_{\epsilon}(y, \sigma)-k\right) g^{2}\left(u_{\epsilon}(y, \sigma)\right) d \sigma\right) d k d y d s\right] \\
= & \mathbb{I}_{1}+\mathbb{I}_{2}+\mathbb{I}_{3} .
\end{aligned}
$$

And, using again the same techniques as in BVW12 (p.689-693), we prove that $\mathbb{I}_{1}$ and $\mathbb{I}_{3}$ tend to 0 as $n \rightarrow \infty$. Let us mention that the regularity $\epsilon \Delta u_{\epsilon}-\operatorname{div}\left[\vec{v}(.,) f.\left(u_{\epsilon}\right)\right] \epsilon L^{2}(\Omega \times Q)$ is exploited to show that $\mathbb{I}_{1} \rightarrow 0($ as $n \rightarrow \infty)$ and that the $L^{4}(\Omega \times Q)$ regularity of $u_{\epsilon}$ given by Proposition 8 is used to show that $\mathbb{I}_{3} \rightarrow 0($ as $n \rightarrow \infty)$. Moreover, thanks to the Itô isometry, we also prove that

$$
\left.\mathbb{I}_{2} \quad \underset{n, l}{\longrightarrow}-E\left[\int_{0}^{1} \int_{Q} \int_{\mathbb{R}^{d}} \eta^{\prime \prime}(\hat{\mathbf{u}}(p))-u_{\epsilon}(y, t)\right) g(\hat{\mathbf{u}}(p)) g\left(u_{\epsilon}(y, t)\right) \varphi(y, t) \rho_{m}(x-y) d y d p\right]
$$

Now, combining the preceding estimates yields that

$$
\begin{aligned}
& \lim _{l} \lim _{n}\left[I_{6}+J_{8}+I_{7}+J_{9}\right] \\
= & \frac{1}{2} E\left[\int_{0}^{1} \int_{Q} \int_{\mathbb{R}^{d}}\left\{g(\hat{\mathbf{u}}(p))-g\left(u_{\epsilon}(y, t)\right)\right\}^{2} \eta^{\prime \prime}\left(u_{\epsilon}(y, t)-\hat{\mathbf{u}}(p)\right) \rho_{m}(x-y) \varphi(y, t) d y d p\right] \\
\vec{\eta} \quad & 0,
\end{aligned}
$$

for $\eta=\eta_{\delta} \in \mathcal{A}$, the approximation of the absolute value function as defined above, since $\operatorname{supp} \eta^{\prime \prime} \subset[-\delta, \delta]$, and $\left|\eta^{\prime \prime}\right| \leqslant \frac{2 \pi}{\delta}$. Finally, passing to the limits in $I_{1}+. .+I_{7}+J_{1}+. .+J_{9}$ successively with $n, l, \eta=\eta_{\delta}, \epsilon$ and $m$, we thus obtain for any function $\varphi$ in $\mathcal{D}^{+}\left(\mathbb{R}^{d} \times[0, T)\right)$

$$
\begin{aligned}
0 \leqslant & E\left[\int_{\mathbb{R}^{d}}\left|\hat{u}_{0}(x)-u_{0}(x)\right| \varphi(x, 0) d x\right]+E\left[\int_{Q} \int_{0}^{1} \int_{0}^{1}|\mathbf{u}(x, t, \beta)-\hat{\mathbf{u}}(x, t, \alpha)| \partial_{t} \varphi(x, t) d \alpha d \beta d x d t\right] \\
+ & E\left[\int_{Q} \int_{0}^{1} \int_{0}^{1} \mathcal{F}(\mathbf{u}(x, t, \beta), \hat{\mathbf{u}}(x, t, \alpha)) \vec{v}(x, t) \nabla_{x} \varphi(x, t) d \alpha d \beta d x d t\right] .
\end{aligned}
$$

Note finally that, thanks to a density argument, this inequality still holds for any nonnegative test-function $\varphi \in H^{1}\left(\mathbb{R}^{d} \times[0, T)\right)$ with a compact support and finally we get the Kato Inequality 59 .

\section{References}

[Bal00] E. J. Balder. Lectures on Young measure theory and its applications in economics. Rend. Istit. Mat. Univ. Trieste, 31(suppl. 1):1-69, 2000. Workshop on Measure Theory and Real Analysis (Italian) (Grado, 1997). 
[Bau14] C. Bauzet. On a time-splitting method for a scalar conservation law with a multiplicative stochastic perturbation and numerical experiments. Journal of Evolution Equations, 14(2): 333-356, 2014.

[BCG] C. Bauzet, J. Charrier, and T. Gallouët. Convergence of flux-splitting finite volume schemes for hyperbolic scalar conservation laws with a multiplicative stochastic perturbation. Submitted, 2014.

[BVW12] C. Bauzet, G. Vallet, and P. Wittbold. The Cauchy problem for a conservation law with a multiplicative stochastic perturbation. Journal of Hyperbolic Differential Equations, 9(4):661709, 2012.

[BVW14] C. Bauzet, G. Vallet, and P. Wittbold. The Dirichlet problem for a conservation law with a multiplicative stochastic perturbation. Journal of Functional Analysis, 4(266):2503-2545, 2014.

[BM14] I.H. Biswas and A.K. Majee. Stochastic conservation laws: Weak-in-time formulation and strong entropy condition. Journal of Functional Analysis, 7(267):2199-2252, 2014.

[CH00] C. Chainais-Hillairet. Second-order finite-volume schemes for a non-linear hyperbolic equation: error estimate. Mathematical Methods in the Applied Sciences, 23(5):467-490, 2000.

[CDK12] G.-Q. Chen, Q. Ding, and K. H. Karlsen. On nonlinear stochastic balance laws. Arch. Ration. Mech. Anal., 204(3):707-743, 2012.

[DPZ92] G. Da Prato and J. Zabczyk. Stochastic equations in infinite dimensions, volume 44 of Encyclopedia of Mathematics and its Applications. Cambridge University Press, Cambridge, 1992.

[DV10] A. Debussche and J. Vovelle. Scalar conservation laws with stochastic forcing. J. Funct. Anal., 259(4):1014-1042, 2010.

[EGH95] R. Eymard, T. Gallouët, and R. Herbin. Existence and uniqueness of the entropy solution to a nonlinear hyperbolic equation. Chinese Ann. Math. Ser. B, 16(1):1-14, 1995. A Chinese summary appears in Chinese Ann. Math. Ser. A 16 (1995), no. 1, 119.

[EGH00] R. Eymard, T. Gallouët, and R. Herbin. Finite volume methods. In Handbook of numerical analysis, Vol. VII, Handb. Numer. Anal., VII, pages 713-1020. North-Holland, Amsterdam, 2000.

[FN08] J. Feng and D. Nualart. Stochastic scalar conservation laws. J. Funct. Anal., 255(2):313-373, 2008.

[GT95] W. Grecksch and C. Tudor. Stochastic evolution equations, volume 85 of Mathematical Research. Akademie-Verlag, Berlin, 1995. A Hilbert space approach.

[Hof14] M. Hofmanová. Bhatnagar-gross-krook approximation to stochastic scalar conservation laws. Ann. Inst. H. Poincare Probab. Statist., 2014.

[HR91] H. Holden and N. H. Risebro. A stochastic approach to conservation laws. In Third International Conference on Hyperbolic Problems, Vol. I, II (Uppsala, 1990), pages 575-587. Studentlitteratur, Lund, 1991.

[Kim06] J.U. Kim. On the stochastic porous medium equation. J. Differential Equations, 220(1):163-194, 2006.

[KR12] I. Kröker and C. Rohde. Finite volume schemes for hyperbolic balance laws with multiplicative noise. Appl. Numer. Math., 62(4):441-456, 2012.

[Pan96] E. Yu. Panov. On measure-valued solutions of the Cauchy problem for a first-order quasilinear equation. Izv. Ross. Akad. Nauk Ser. Mat., 60(2):107-148, 1996.

[Val08] G. Vallet. Stochastic perturbation of nonlinear degenerate parabolic problems. Differential Integral Equations, 21(11-12):1055-1082, 2008. 